\title{
DUALITY THEOREMS IN DEFORMATION THEORY
}

\author{
BY \\ HUBERT GOLDSCHMIDT ${ }^{1}$
}

\begin{abstract}
We give a unified treatment of the construction of the Calabi sequence, which is a resolution of the sheaf of Killing vector fields on a Riemannian manifold of constant curvature, and of the resolution of the sheaf of conformal Killing vector fields on a conformally flat Riemannian manifold of dimension $\geqslant 3$ introduced in [7]. We also explain why the latter resolution is selfadjoint and associate to certain geometric structures selfadjoint resolutions of their infinitesimal automorphisms.
\end{abstract}

Introduction. In the deformation theory of pseudogroup structures as developed by K. Kodaira and D. C. Spencer $[\mathbf{2 1}, \mathbf{2 0}, \mathbf{2 3}]$, one of the fundamental problems consists of interpreting the space of infinitesimal deformations of a geometric structure on a compact manifold $X$ of dimension $n$, which is isomorphic to the first cohomology group $H^{1}(X, \Theta)$ of $X$ with values in the sheaf $\Theta$ of infinitesimal automorphisms of the geometric structure, and then of determining which elements of $H^{1}(X, \Theta)$ correspond to an actual deformation of the structure. Certain classes in $H^{1}(X, \Theta)$ may be obstructed, the obstructions occurring as classes in $H^{2}(X, \Theta)$.

If the automorphisms of the structure form an analytic and elliptic pseudogroup, according to [23 and 17], the Spencer sequence

$$
0 \rightarrow \Theta \rightarrow \mathscr{C}^{0} \stackrel{D}{\rightarrow} \mathscr{C}^{1} \stackrel{D}{\rightarrow} \mathscr{C}^{2} \stackrel{D}{\rightarrow} \cdots \rightarrow \mathscr{C}^{n} \rightarrow 0
$$

is a fine resolution of $\Theta$ and an elliptic complex. Here $\mathscr{C}^{j}$ is the sheaf of $C^{\infty}$-sections of a vector bundle $C^{j}$ over $X$ and $D$ is a first-order differential operator, and $\mathscr{C}=\oplus_{j} \mathscr{C}^{j}$ possesses the structure of a graded Lie algebra which is compatible with $D$, i.e.

$$
D[u, v]=[D u, v]+(-1)^{i}[u, D v]
$$

for $u \in \mathscr{C}^{i}, v \in \mathscr{C}^{j}$. Moreover, the space $H^{j}(X, \Theta)$ is finite-dimensional and isomorphic to the cohomology of the sequence

$$
\Gamma\left(X, \mathscr{C}^{j-1}\right) \stackrel{D}{\rightarrow} \Gamma\left(X, \mathscr{C}^{j}\right) \stackrel{D}{\rightarrow} \Gamma\left(X, \mathscr{C}^{j+1}\right) ;
$$

Received by the editors July 29, 1983 and, in revised form, March 5, 1984.

1980 Mathematics Subject Classification. Primary 58G05, 58H15; Secondary 17B56, 17B70, 53A30.

Key words and phrases. Selfadjoint resolution, space of constant curvature, conformally flat Riemannian manifold, semisimple Lie algebra, differential operator, spectral sequence.

${ }^{1}$ The author was supported in part by National Science Foundation Grant MCS-8108814(A01). 
if a cohomology class in $H^{1}(X, \Theta)$ is represented by a section $u$ of $C^{1}$ over $X$ satisfying $D u=0$, the obstruction to finding a deformation tangent to this class lies in $H^{2}(X, \Theta)$ and corresponds to the cohomology class of the section $\frac{1}{2}[u, u]$ of $C^{2}$.

For complex structures, this is an extremely satisfactory theory. Unfortunately, for certain other structures this theory does not always lead to explicit computations and alternative interpretations of the cohomology groups $H^{j}(X, \Theta)$ must be found. Here, we are interested in structures giving rise to pseudogroups of finite type. Then $\Theta$ is a locally constant sheaf and the Spencer sequence is isomorphic to the canonical resolution of $\Theta$, which is a twisted de Rham complex. In certain cases, one can construct other fine resolutions of $\Theta$, whose bundles and differential operators can be explicitly described by means of the geometric structure, and in terms of which the infinitesimal deformations have a more geometric interpretation. Two resolutions of this type have been constructed, namely by Calabi [2] for the sheaf of Killing vector fields on a Riemannian manifold of constant curvature and in [7] for the sheaf of conformal Killing vector fields on a conformally flat Riemannian manifold of dimension $\geqslant 3$. In both these cases, an element of $H^{1}(X, \Theta)$ can be represented by a variation of the metric, which is a symmetric 2 -form. Using these sequences, for certain spaces one can compute the space of infinitesimal deformations. For example, Calabi proved that $H^{1}(X, \Theta)$ vanishes when $\Theta$ is the sheaf of Killing vector fields on a compact quotient of hyperbolic $n$-space, with $n \geqslant 3$. In [7, §17], all the cohomology groups $H^{j}(X, \Theta)$ are computed for the sheaf $\Theta$ of Killing or of conformal Killing vector fields on a flat torus. Since a good obstruction theory does not as yet exist in the context of these sequences, it is important to relate explicitly the Spencer sequence to the geometric resolution for these two structures in order to carry out computations for certain examples, in particular for the conformally flat structures on compact quotients of hyperbolic space (see [22]).

In this paper, we propose to give a unified treatment of the construction of these two geometric resolutions by means of a spectral sequence, which permits us to associate directly to a section $u$ of $C^{j}$ satisfying $D u=0$ a section of the $(j+1)$ th vector bundle of the geometric resolution corresponding to the same cohomology class in $H^{\prime}(X, \Theta)$. This should enable us to relate the deformation and obstruction theory given in terms of the Spencer sequence to one worked out in terms of the geometric resolution.

If $g$ is a conformally flat Riemannian metric on $X$, an infinitesimal deformation of the corresponding conformal structure is represented by a section $u$ of the bundle $C^{1}$ of the Spencer sequence satisfying $D u=0$. It determines an infinitesimal variation $h$ of the metric $g$, which is a symmetric 2 -form. If $n \geqslant 4$, we show here that the condition $D u=0$ implies that the linearization of the Weyl tensor operator vanishes on $h$, that is, $h$ is a cocycle of the geometric sequence of [7]. The second order variation of the Weyl tensor operator gives us a section of a bundle of this sequence, which is a cocycle and which has an explicit quadratic expression in terms of $h$ and its first derivatives. Using the methods developed here, we hope to prove that this section corresponds to the cohomology class of the section $\frac{1}{2}[u, u]$ of $C^{2}$ in $H^{2}(X, \Theta)$. Using the Spencer sequence and cur spectral sequence, we explain in 
algebraic terms why the duality theorem for the cohomology groups $H^{j}(X, \Theta)$ established in [7] holds and why the geometric resolution of $\Theta$ is selfadjoint.

We denote by $T$ and $T^{*}$ the tangent and cotangent bundles of $X$ and by $\mathscr{E}$ the sheaf of sections of a vector bundle $E$ over $X$. Let $R_{m}$ be a formally integrable linear differential equation of order $m$ on a vector bundle $E$ over $X$ corresponding to the homogeneous equation $P s=0$, for $s \in \mathscr{E}$, where $P$ is a linear differential operator of order $m$. Assume that $R_{m}$ is of finite type, in particular that the first prolongation of its symbol vanishes. Then a solution $s$ of $R_{m}$ or of the equation $P u=0$ over a connected open subset $U$ of $X$ is uniquely determined by its $m$-jet at $x \in U$, and the sheaf $\Theta$ of solutions of $R_{m}$ is locally constant and admits a canonical resolution

$$
0 \rightarrow \Theta \rightarrow \mathscr{R}_{m} \stackrel{\nabla^{*}}{\rightarrow} \mathscr{T}^{*} \otimes \mathscr{R}_{m} \stackrel{\nabla^{\cdot}}{\rightarrow} \wedge^{2} \mathscr{T}^{*} \otimes \mathscr{R}_{m} \rightarrow \cdots \rightarrow \wedge^{n} \mathscr{T}^{*} \otimes \mathscr{R}_{m} \rightarrow 0,
$$

which is a twisted de Rham sequence. We suppose that $R_{m}$ satisfies some regularity conditions and in particular that the Spencer cohomology groups $H^{p, j}\left(R_{m}\right)$ of $R_{m}$, with $0 \leqslant j \leqslant n, 0 \leqslant p \leqslant m$, defined in $\S 3$ are vector bundles. The vector bundle $R_{m}$ is filtered and this permits us to define a filtration $\left\{\mathscr{C}^{p}\right\}$ of $\wedge \mathscr{T}^{*} \otimes \mathscr{R}_{m}$ determined by sub-bundles $C^{p}$ stable under the differential $\nabla^{\wedge}$. We consider the corresponding spectral sequence $\left\{E_{r}^{p, j}, d_{r}\right\}$, and verify that $E_{0}^{2 p, j}$ is canonically isomorphic to the sheaf of sections of $H^{p, j}\left(R_{m}\right)$ and that $E_{1}^{2 p-1, j}=0$. If there exist integers

$$
0=r_{0} \leqslant r_{1} \leqslant \cdots \leqslant r_{n-1} \leqslant r_{n} \leqslant m
$$

such that $H^{p, j}\left(R_{m}\right)=0$ for all $p \neq r_{j}$, with $0 \leqslant j \leqslant n$ and $0 \leqslant p \leqslant m$, then, because of the vanishing of appropriate $E_{0}$ and $E_{1}$ terms of this spectral sequence, from the sequence (1) we obtain a complex

$$
0 \rightarrow \Theta \rightarrow \mathscr{E}^{0} \stackrel{D^{0}}{\rightarrow} \mathscr{E}^{1} \stackrel{D^{1}}{\rightarrow} \mathscr{E}^{2} \stackrel{D^{2}}{\rightarrow} \cdots \rightarrow \mathscr{E}^{n} \rightarrow 0
$$

where $E^{j}=H^{r_{j}, j}\left(R_{m}\right)$ and $D^{j}$ is a differential operator of order $r_{j+1}-r_{j}+1$ derived from the differential $d_{2\left(r_{1+1}-r_{1}\right)}$ of the spectral sequence; using results from the theory of spectral sequences, we prove that this sequence is exact and an elliptic complex. Moreover, we always have $H^{0,0}\left(R_{m}\right)=E^{0}=E$.

In [5], it is shown that the first-order equation $R_{1}$ for the Killing vector fields of a Riemannian manifold $(X, g)$ of constant curvature satisfies all the above conditions with $m=1, r_{0}=r_{1}=0$ and $r_{2}=\cdots=r_{n}=1$; we thus have the resolution (2) of the sheaf $\Theta$ of Killing vector fields of $(X, g)$, where $D_{0}, D_{j}$ (with $j \geqslant 2$ ) are differential operators of order 1 and $D_{1}$ is of order 2. There are canonical isomorphisms

$$
T \rightarrow H^{0,0}\left(R_{1}\right), \quad S^{2} T^{*} \rightarrow H^{0,1}\left(R_{1}\right), \quad E_{j-1} \rightarrow H^{1, j}\left(R_{1}\right)
$$

for $2 \leqslant j \leqslant n$, where $S^{2} T^{*}$ is the bundle of symmetric 2-forms on $X$ and $E_{j-1}$ is a sub-bundle of $\wedge^{j} T^{*} \otimes \wedge^{2} T^{*}$. By means of these isomorphisms, from the resolution (2) of $\Theta$, we obtain a resolution

$$
0 \rightarrow \Theta \rightarrow \mathscr{T}^{Q_{0}} \rightarrow S^{2} \mathscr{T}^{*} \stackrel{Q_{1}}{\rightarrow} \mathscr{E}_{1} \stackrel{Q_{2}}{\rightarrow} \mathscr{E}_{2} \rightarrow \cdots \rightarrow \mathscr{E}_{n-1} \rightarrow 0 .
$$


In $\S 6$, we show that it is the Calabi sequence of [2] as defined in [5]; in particular, if $\xi$ is a vector field on $X$, we verify that $Q_{0} \xi$ is the Lie derivative of $g$ along $\xi$.

If $\nabla$ is a torsionless connection on $X$ and $(X, \nabla)$ is an affinely flat manifold, then the equation $R_{2}$ of order 2 for the infinitesimal affine transformations also satisfies all the above conditions with $m=2, r_{0}=0$ and $r_{1}=\cdots=r_{n}=1$, and so we have the resolution (2) of the sheaf $\Theta$ of infinitesimal affine transformations of $(X, \nabla)$, where $D_{0}$ is a differential operator of order 2 and $D_{j}$ (with $j \geqslant 1$ ) is of order 1 which can be explicitly described in terms of $\nabla$.

We now suppose that $R_{m}$ is a Lie equation in the tangent bundle of $X$. Then $R_{m . x}$ has the structure of a Lie algebra for $x \in X$. In terms of the Killing forms of these Lie algebras, we define a pairing

$$
\left(\wedge^{i} T^{*} \otimes R_{m}\right) \otimes\left(\wedge^{j} T^{*} \otimes R_{m}\right) \rightarrow \wedge^{i+j} T^{*},
$$

sending $u \otimes v$ into $\langle u, v\rangle$, such that

$$
\langle\nabla \hat{u}, v\rangle+(-1)^{i}\langle u, \nabla \hat{v}\rangle=d\langle u, v\rangle
$$

for $u \in \wedge^{i} \mathscr{T}^{*} \otimes \mathscr{R}_{m}, v \in \wedge^{j} \mathscr{T}^{*} \otimes \mathscr{R}_{m}$. If $H_{0}^{k}(X, \Theta)$ denotes the $k$ th cohomology group of $X$ with compact support and values in $\Theta$, then, from this pairing and the resolution (1), when $X$ is oriented we obtain a pairing

$$
H^{j}(X, \Theta) \otimes H_{0}^{n-j}(X, \Theta) \rightarrow \mathbf{R} .
$$

Whenever $R_{m . x}$ is a semisimple Lie algebra for all $x \in X$, we prove that this pairing is nondegenerate (Theorem 1.1). We assume henceforth that $m=2$. By restriction and passage to the quotient, the pairing (3) induces a pairing

$$
H^{p, i}\left(R_{m}\right) \otimes H^{2-p . j}\left(R_{m}\right) \rightarrow \wedge^{i+j} T^{*}
$$

for $0 \leqslant p \leqslant 2$, sending $u \otimes v$ into $\langle u, v\rangle$. Suppose that there exists an integer $0 \leqslant k \leqslant(n+1) / 2$ such that

$$
\begin{array}{ll}
H^{0 . j}\left(R_{2}\right)=0 & \text { for } j>k, \\
H^{1, j}\left(R_{2}\right)=0 & \text { for } j \leqslant k \text { and } j \geqslant n-k, \\
H^{2 . j}\left(R_{2}\right)=0 & \text { for } j<n-k ;
\end{array}
$$

then the condition described above for the existence of the sequence (2) is verified with $r_{i}=0$ for $0 \leqslant i \leqslant k, r_{j}=1$ for $k+1 \leqslant j \leqslant n-k-1$, and $r_{l}=2$ for $n-k \leqslant$ $l \leqslant n$. We have $r_{n-j}=2-r_{j}$ for $0 \leqslant j \leqslant n$ and we prove that there exists a differential operator

$$
\alpha_{j}: \mathscr{E}^{j} \oplus \mathscr{E}^{n-j-1} \rightarrow \wedge^{n-1} \mathscr{T}^{*}
$$

of order $r_{j+1}-r_{j}$ such that

$$
\left\langle D_{j} u, v\right\rangle+(-1)^{j}\left\langle u, D_{n-j-1} v\right\rangle=d \alpha_{j}(u \oplus v)
$$

for all $u \in \mathscr{E}^{j}, v \in \mathscr{E}^{n-j-1}$. Thus when $X$ is oriented, from the resolution (2) and the pairing (5) we obtain another pairing

$$
H^{j}(X, \Theta) \otimes H_{0}^{n-j}(X, \Theta) \rightarrow \mathbf{R} ;
$$


by means of our spectral sequence, we show that this pairing is indeed equal to (4) (Theorem 5.1).

For $x \in X$, the fiber $R_{2, x}$ is a filtered Lie algebra and thus the fibers of the graded bundle $g=g_{0} \oplus g_{1} \oplus g_{2}$ associated to the filtered bundle are also Lie algebras. If $g_{x}$ is semisimple for $x \in X$, so is $R_{2, x}$ and the pairing (4) is therefore nondegenerate whenever $X$ is oriented; moreover, there exists a morphism $\sigma: g \rightarrow g$ of vector bundles such that $\sigma_{x}$ is a Cartan involution of $g_{x}$ for all $x \in X$, and

$$
\sigma\left(g_{p}\right)=g_{2-p}
$$

for $p=0,1,2$. The Killing forms of the Lie algebras $g_{x}$ and $\sigma$ determine a scalar product on the bundle $g$ and a metric on $X$. Using the induced scalar product ( , ) on $\wedge T^{*} \otimes g$, we construct a sub-bundle $F^{j}$ of $\wedge^{j} T^{*} \otimes g_{r_{i}}$ and an isomorphism $\gamma$ : $F^{j} \rightarrow E^{j}$. The pairings (5) determine morphisms

$$
F^{j} \otimes F^{n-j} \rightarrow \wedge^{n} T^{*}
$$

sending $u \otimes v$ into $\langle u, v\rangle=\langle\gamma u, \gamma v\rangle$. By means of the isomorphisms $\gamma$, from the sequence (2) and (6) we obtain a resolution

$$
0 \rightarrow \Theta \rightarrow \mathscr{F}^{0} \stackrel{P^{0}}{\rightarrow} \mathscr{F}^{1} \stackrel{P^{1}}{\rightarrow} \mathscr{F}^{2} \stackrel{P^{2}}{\rightarrow} \cdots \rightarrow \mathscr{F}^{n} \rightarrow 0
$$

of $\Theta$ and differential operators

$$
\tilde{\alpha}_{j}: \mathscr{F}^{j} \oplus \mathscr{F}^{n-j-1} \rightarrow \wedge^{n-1} \mathscr{T}^{*}
$$

of order $r_{j+1}-r_{j}$ which have properties similar to those of the de Rham sequence, namely:

(I) For $u \in \mathscr{F}^{j}$ and $v \in \mathscr{F}^{n-j-1}$, we have

$$
\left\langle P^{j} u, v\right\rangle+(-1)^{j}\left\langle u, P^{n-j-1} v\right\rangle=d \tilde{\alpha}_{j}(u \oplus v) .
$$

(II) If $X$ is oriented, then because of (7), the isomorphisms

$$
*: \wedge^{j} T^{*} \otimes g_{r_{i}} \rightarrow \wedge^{n-j} T^{*} \otimes g_{r_{n-j}},
$$

defined in terms of the Hodge $*$-isomorphism by

$$
*(\alpha \otimes \xi)=-(* \alpha) \otimes \sigma(\xi),
$$

for $\alpha \in \wedge^{j} T^{*}, \xi \in g_{r_{i}}$, give us by restriction isomorphisms $*: F^{j} \rightarrow F^{n-j}$ such that

$$
\begin{aligned}
& * * u=(-1)^{n j+j} u, \\
& \langle u, * v\rangle=(u, v) \Omega
\end{aligned}
$$

for $u, v \in F^{j}$, where $\Omega$ is the volume form corresponding to the metric on $X$.

(III) If $X$ is oriented, the formal adjoint $P^{j *}: \mathscr{F}^{j+1} \rightarrow \mathscr{F}^{j}$ of $P^{j}$ is equal to $(-1)^{n j+j} * P^{n-j-1} *$.

(IV) The Laplacians $\square^{j}: \mathscr{F}^{j} \rightarrow \mathscr{F}^{j}$ defined by

$$
\square^{j}=\left(P^{j-1} P^{j-1 *}\right)^{r_{j+1}-r_{j}+1}+\left(P^{j *} P^{j}\right)^{r_{j}-r_{j-1}+1}
$$

are formally selfadjoint and elliptic and, if $X$ is oriented, satisfy the relations

$$
* \square^{j}=\square^{n-j} * \text {. }
$$


These properties of the sequence (8) imply that it is formally selfadjoint; hence the adjoint complex

$$
0 \rightarrow 0 \otimes \mathbf{Z}^{\Theta} \rightarrow \mathscr{F}^{n} \stackrel{P^{n-1 *}}{\rightarrow} \mathscr{F}^{n-1}{\stackrel{P^{n}}{\rightarrow}}^{2 *} \cdots \rightarrow \mathscr{F}^{1} \stackrel{P^{0 *}}{\rightarrow} \mathscr{F}^{0} \rightarrow 0
$$

of (7) is exact, where $o$ is the orientation sheaf of $X$ (over $\mathbf{Z}$ ).

In $§ 7$, we study the first-order differential equation $R_{1}$ for the conformal Killing vector fields of a conformally flat Riemannian manifold $(X, g)$ of dimension $n \geqslant 3$. Using results of [7], we verify that the first prolongation $R_{2}$ of this equation satisfies all the above assumptions with $m=2$ and $k=2$, and that $R_{2, x}$ is a simple Lie algebra for all $x \in X$. We thus have the resolution (2) of the sheaf $\Theta$ of conformal Killing vector fields of $(X, g)$, where $D_{j}$, with $j \neq 1, n-2$, are differential operators of order $1, D_{1}, D_{n-2}$ are of order 2 when $n \geqslant 4$, and $D_{1}$ is of order 3 when $n=3$. There are isomorphisms of vector bundles depending on $g$

$$
\begin{gathered}
\varphi_{0}: T \rightarrow H^{0,0}\left(R_{2}\right), \quad \varphi_{1}: S_{0}^{2} T^{*} \rightarrow H^{0,1}\left(R_{2}\right), \\
\varphi_{j}: E_{j-1}^{0} \rightarrow H^{1, j}\left(R_{2}\right), \\
\varphi_{n-1}: \wedge^{n} T^{*} \otimes S_{0}^{2} T^{*} \rightarrow H^{2, n-1}\left(R_{2}\right), \quad \varphi_{n}: \wedge^{n} T^{*} \otimes T^{*} \rightarrow H^{2, n}\left(R_{2}\right),
\end{gathered}
$$

where $S_{0}^{2} T^{*}$ is the sub-bundle of $S^{2} T^{*}$ of 2 -forms with zero trace, and $E_{j-1}^{0}$ is a sub-bundle of $\wedge^{j} T^{*} \otimes \wedge^{2} T^{*}$ for $2 \leqslant j \leqslant n-2$, such that the resolution of $\Theta$ obtained from (2) by means of these isomorphisms is the sequence

$$
0 \rightarrow \Theta \rightarrow \mathscr{F}_{0} \stackrel{P_{0}}{\rightarrow} \mathscr{F}_{1} \stackrel{P_{1}}{\rightarrow} \mathscr{F}_{2} \stackrel{P_{2}}{\rightarrow} \cdots \rightarrow \mathscr{F}_{n} \rightarrow 0
$$

of [7]. From the relations (6), we deduce the results given by Propositions 11.1 and 11.2 of [7]. We then show that the nondegenerate pairing (4) obtained from (5) is equal to $(-c n)$-times the corresponding pairing described in [7] when $X$ is compact, where $c=8$ if $n \geqslant 4$ and $c=2$ if $n=3$ (Theorem 7.1). Moreover, if $\Omega^{-2 / n}$ denotes the bundle of densities of order $-2 / n$ on $X$, there exist isomorphisms of vector bundles

$$
\tilde{\varphi}_{0}=\varphi_{0}: F_{0} \rightarrow E^{0}, \quad \tilde{\varphi}_{n}=\varphi_{n}: F_{n} \rightarrow E^{n}, \quad \tilde{\varphi}_{j}: \Omega^{-2 / n} \otimes F_{j} \rightarrow E^{j}
$$

for $1 \leqslant j \leqslant n-1$, depending only on the conformal class of $g$, such that the resolution of $\Theta$ obtained from (2) by means of these isomorphisms is the sequence (13.14) of [7], and we thus show that this complex depends only on the conformal class of $g$, a result verified in $[7, \S 13]$.

In terms of the metric, we define a Cartan involution of the graded bundle $g$ satisfying (7); consider the corresponding scalar product $(, \quad)$ on $\wedge T^{*} \otimes g$ and the sub-bundle $F^{j}$ of $\wedge^{j} T^{*} \otimes g_{r_{j}}$. We define an isomorphism $\psi_{j}: F_{j} \rightarrow F^{j}$ such that $\gamma \psi_{j}=\varphi_{j}$. If $X$ is oriented, then, in terms of the morphisms $*: F^{j} \rightarrow F^{n-j}$ determined by $\sigma$, the morphism $*: F_{j} \rightarrow F_{n-j}$ defined in [7] is equal to $d_{j} \psi_{n-j}^{-1} * \psi_{j}$, where $d_{j}$ is a constant depending only on $n$ and $j$. From (9) and (10), we deduce the corresponding relations for the isomorphisms $*: F_{j} \rightarrow F_{n-j}$ of [7]; thus we have shown that the resolution (11) of $\Theta$ is a formally selfadjoint complex, namely, that it satisfies conditions (I)-(IV) of the introduction of [7]. 
If $\nabla$ is a torsionless connection on $X$ and $n \geqslant 2$, and if $(X, \nabla)$ is a projectively flat manifold, then the second order differential equation $R_{2}$ for the infinitesimal projective transformations satisfies all the above assumptions with $m=2$ and $k=1$, and $R_{2, x}$ is a simple Lie algebra for all $x \in X$. Thus we have the resolution (2) of the sheaf $\Theta$ of infinitesimal projective transformations of $(X, \nabla)$, where $D_{j}$, with $j \neq 0$, $n-1$, are differential operators of order 1 and $D_{0}, D_{n-1}$ are of order 2. Moreover, we have the nondegenerate pairing (4) and the sequence (2) is formally selfadjoint. The operators of this sequence can be explicitly described in terms of $\nabla$, although we do not carry out this computation here.

Finally, we make some additional comments about the contents of this paper. $\$ 1$ contains some results from the theory of overdetermined differential operators of [8 and 17], namely formula (1.13) which is needed to establish (3.6) and the commutativity of (3.5); these are the two facts which permit us to construct our spectral sequence in $\S 3$. In $\S 1$, we also provide two proofs of the nondegeneracy of the pairing (4). In $\S 2$, we present the results on spectral sequences, which are required in $\$ 3$ to construct and prove the exactness of sequence (2), and which are similar to certain ones of [3, Chapter XV]. In $\S 3$, we define our filtration $\left\{\mathscr{C}^{p}\right\}$ and our spectral sequence, and give explicit formulas for the differential operators of the sequence (2) and their symbols. We also prove the commutativity of diagram (3.20), from which we deduce Theorem 5.1. $\S 4$ is devoted to the structure of semisimple graded Lie algebras; we establish there a duality result for their cohomology (Proposition 4.4), which is proved in a special case in [7].

We wish to thank J. C. Moore, E. Van den Ban, J. L. Verdier and G. Zuckerman for the most helpful discussions concerning this paper.

1. A duality theorem. Let $X$ be a differentiable manifold of dimension $n$ and class $C^{\infty}$, whose tangent bundle we denote by $T$. We write $\mathcal{O}_{X}$ for the sheaf of real-valued, differentiable functions on $X$. Let $S^{\prime} T^{*}$ and $\wedge^{m} T^{*}$ be the $l$ th symmetric power of $T^{*}$ and the $m$ th exterior product of $T^{*}$, and let $\wedge T^{*}$ be the exterior algebra of $T^{*}$. Let $E$ be a vector bundle over $X$; we denote by $\mathscr{E}$ the sheaf of $C^{\infty}$-sections of $E$ and by $J_{k}(E)$ the bundle of $k$-jets of sections of $E$. We write $C^{\infty}(E)$ (resp. $C_{0}^{\infty}(E)$ ) for the space of $C^{\infty}$-sections of $E$ (resp. of $E$ with compact support) over $X$. Let $j_{k}$ : $\mathscr{E} \rightarrow J_{k}(\mathscr{E})$ be the differential operator of order $k$ which sends a section $s$ of $E$ over a neighborhood of $x \in X$ into the $k$-jet $j_{k}(s)$ of this section and let $\pi_{k}: J_{k+l}(E) \rightarrow J_{k}(E)$ be the natural projection sending $j_{k+1}(s)(x)$ into $j_{k}(s)(x)$. We identify $J_{0}(E)$ with $E$ and we set $J_{k}(E)=0$ for $k<0$. We have a natural injection

$$
\lambda_{l}: J_{k+l}(E) \rightarrow J_{l}\left(J_{k}(E)\right) \text {, }
$$

which sends $j_{k+l}(s)(x)$ into $j_{l}\left(j_{k}(s)\right)(x)$, and an exact sequence of vector bundles

$$
0 \rightarrow S^{k} T^{*} \otimes E \stackrel{\varepsilon}{\rightarrow} J_{k}(E) \stackrel{\pi_{k-1}}{\rightarrow} J_{k-1}(E) \rightarrow 0,
$$

where $\varepsilon$ is the morphism defined as follows: if $f_{1}, \ldots, f_{k}$ are real-valued functions on a neighborhood of $x$ which vanish at $x$, then

$$
\varepsilon\left(\left(d f_{1} \cdot \cdots \cdot d f_{k}\right)(x) \otimes s(x)\right)=j_{k}\left(\left(\prod_{i=1}^{k} f_{i}\right) s\right)(x) .
$$


The sequence (1.1) yields the exact sequence

$$
0 \rightarrow T^{*} \otimes J_{k-1}(E) \stackrel{\varepsilon}{\rightarrow} J_{1}\left(J_{k-1}(E)\right) \stackrel{\pi_{0}}{\rightarrow} J_{k-1}(E) \rightarrow 0 .
$$

If $F$ is another vector bundle over $X$ and $Q: \mathscr{E} \rightarrow \mathscr{F}$ is a differential operator of order $k$, then $Q$ induces a unique morphism of vector bundles $p(Q): J_{k}(E) \rightarrow F$ such that $p(Q) \circ j_{k}=Q$; the symbol $\sigma(Q): S^{k} T^{*} \otimes E \rightarrow F$ of $Q$ is the morphism $p(Q) \circ \varepsilon$.

We define a first-order differential operator

$$
D: J_{k}(\mathscr{E}) \rightarrow \mathscr{T}^{*} \otimes J_{k-1}(\mathscr{E})
$$

by

$$
\varepsilon D u=j_{1}\left(\pi_{k-1} u\right)-\lambda_{1} u
$$

for $u \in J_{k}(\mathscr{E})$, and obtain the first-order differential operator

$$
D: \wedge^{j \mathscr{T}^{*}} \otimes J_{k}(\mathscr{E}) \rightarrow \Lambda^{j+1} \mathscr{T}^{*} \otimes J_{k-1}(\mathscr{E})
$$

determined by

$$
D(\omega \otimes u)=d \omega \otimes \pi_{k-1} u+(-1)^{j} \omega \wedge D u
$$

for $\omega \in \wedge^{j} \mathscr{T}^{*}$ and $u \in J_{k}(\mathscr{E})$. We thus obtain the Spencer complex

$$
\begin{aligned}
0 & \rightarrow \mathscr{E} \stackrel{j_{k}}{\rightarrow} J_{k}(\mathscr{E}) \stackrel{D}{\rightarrow} \mathscr{T}^{*} \otimes J_{k-1}(\mathscr{E}) \stackrel{D}{\rightarrow} \wedge^{2} \mathscr{T}^{*} \otimes J_{k-2}(\mathscr{E}) \\
& \stackrel{D}{\rightarrow} \cdots \rightarrow \Lambda^{n} \mathscr{T}^{*} \otimes J_{k-n}(\mathscr{E}) \rightarrow 0,
\end{aligned}
$$

which is an exact sequence. Let $\delta: S^{k+1} T^{*} \rightarrow T^{*} \otimes S^{k} T^{*}$ be the natural inclusion sending $\alpha_{1} \cdot \cdots \cdot \alpha_{k+1}$, with $\alpha_{1}, \ldots, \alpha_{k+1} \in T^{*}$, into

$$
\sum_{j=1}^{k+1} \alpha_{j} \otimes \alpha_{1} \cdot \cdots \cdot \hat{\alpha}_{j} \cdot \cdots \cdot \alpha_{k+1} ;
$$

for $u \in S^{k+1} T^{*}$ and $\xi \in T$, we denote by $\delta_{\xi} u$ the element of $S^{k} T^{*}$ defined by

$$
\delta_{\xi} u=\langle\xi, \delta u\rangle \text {. }
$$

We extend $\delta$ to a morphism of vector bundles

$$
\delta: \wedge^{j} T^{*} \otimes S^{k+1} T^{*} \rightarrow \wedge^{j+1} T^{*} \otimes S^{k} T^{*}
$$

sending $\omega \otimes u$ into $(-1)^{j} \omega \wedge \delta u$, for $\omega \in \wedge^{j} T^{*}$ and $u \in S^{k+1} T^{*}$. Then we have

$$
\delta(\omega \wedge u)=(-1)^{j} \omega \wedge \delta u
$$

for $\omega \in \wedge^{j} T^{*}$ and $u \in \wedge T^{*} \otimes S^{k+1} T^{*}$, and the diagram

$$
\begin{aligned}
& \wedge^{j \mathscr{T}^{*}} \otimes S^{k+1} \mathscr{T}^{*} \otimes \mathscr{E} \quad \stackrel{-\delta}{\rightarrow} \Lambda^{j+1} \mathscr{T}^{*} \otimes S^{k} \mathscr{T}^{*} \otimes \mathscr{E} \\
& \downarrow \varepsilon \\
& \Lambda^{j \mathscr{T}^{*}} \otimes J_{k+1}(\mathscr{E}) \quad \stackrel{D}{\rightarrow} \quad \Lambda^{j+1} \mathscr{T}^{*} \otimes J_{k}(\mathscr{E}) \\
& \downarrow \pi_{k} \quad \downarrow \pi_{k-1} \\
& \bigwedge^{j} \mathscr{T}^{*} \otimes J_{k}(\mathscr{E}) \quad \stackrel{D}{\rightarrow} \quad \Lambda^{j+1} \mathscr{T}^{*} \otimes J_{k-1}(\mathscr{E})
\end{aligned}
$$

commutes (see $[\mathbf{8}, \mathbf{1 7}])$. 
If $R_{k} \subset J_{k}(E)$ is a differential equation of order $k$ on $E$, the symbol of $R_{k}$ is $g_{k}=\left\{u \in S^{k} T^{*} \otimes E \mid \varepsilon u \in R_{k}\right\}$. Now let $R_{k} \subset J_{k}(E)$ be a formally integrable differential equation of order $k$ on $E$, whose $l$ th prolongation is the sub-bundle $R_{k+l}$ of $J_{k+1}(E)$; let $g_{k+1} \subset S^{k+l} T^{*} \otimes E$ be the $l$ th prolongation of the symbol $g_{k}$ of $R_{k}$. Then the differential operator (1.3) gives us by restriction a first-order differential operator

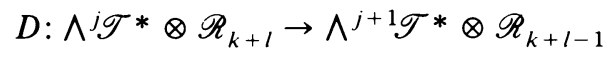

and, according to the commutativity of (1.7), the diagram

$$
\begin{array}{ccc}
\wedge^{j \mathscr{T}^{*}} \otimes g_{k+l} & \stackrel{-\delta}{\rightarrow} & \wedge^{j+1} \mathscr{T}^{*} \otimes g_{k+l-1} \\
\downarrow \varepsilon & & \downarrow \varepsilon \\
\wedge^{j \mathscr{T}^{*}} \otimes \mathscr{R}_{k+l} & \stackrel{D}{\rightarrow} & \wedge^{j+1} \mathscr{T}^{*} \otimes \mathscr{R}_{k+l-1}
\end{array}
$$

commutes. From the exactness of (1.5), we see that, if $\Theta$ denotes the sheaf of solutions of $R_{k}$, the sequence

$$
0 \rightarrow \Theta^{j_{k+1}} \rightarrow \mathscr{R}_{k+1} \stackrel{D}{\rightarrow} \mathscr{T}^{*} \otimes J_{k+l-1}(\mathscr{E})
$$

is exact.

We obtain a complex

$$
\begin{aligned}
0 \rightarrow g_{k+1} & \stackrel{\delta}{\rightarrow} T^{*} \otimes g_{k+l-1} \stackrel{\delta}{\rightarrow} \wedge^{2} T^{*} \otimes g_{k+l-2} \stackrel{\delta}{\rightarrow} \cdots \rightarrow \wedge^{\prime} T^{*} \otimes g_{k} \\
& \stackrel{\delta}{\rightarrow} \wedge^{l+1} T^{*} \otimes S^{k-1} T^{*} \otimes E ;
\end{aligned}
$$

the Spencer cohomology $H^{k+l-j, j}\left(g_{k}\right)$, with $l \geqslant j$, is the cohomology of (1.9) at $\wedge^{i} T^{*} \otimes g_{k+l-j}$. We recall that

$$
H^{k+1,0}\left(g_{k}\right)=0, \quad H^{k+l, 1}\left(g_{k}\right)=0
$$

for $l \geqslant 0$.

Now assume that there exists an integer $m \geqslant k$ such that $g_{m+1}=0$. Then $\pi_{m}$ : $R_{m+1} \rightarrow R_{m}$ is an isomorphism; let $\chi: R_{m} \rightarrow R_{m+1}$ denote its inverse. The composition

$$
R_{m} \stackrel{\chi}{\rightarrow} R_{m+1} \stackrel{\lambda_{1}}{\rightarrow} J_{1}\left(R_{m}\right)
$$

is a connection on $R_{m}$, since $\pi_{0} \lambda_{1} \chi=\mathrm{id}$; it corresponds to the first-order differential operator $\nabla: \mathscr{R}_{m} \rightarrow \mathscr{T}^{*} \otimes \mathscr{R}_{m}$ determined by

$$
\varepsilon \nabla u=j_{1}(u)-\lambda_{1} \chi(u)
$$

for $u \in \mathscr{R}_{m}$. According to (1.2), we see that

$$
\nabla=D \cdot \chi: \mathscr{R}_{m} \rightarrow \mathscr{T}^{*} \otimes \mathscr{R}_{m} .
$$

If

$$
\nabla^{\wedge}: \wedge^{j \mathscr{T}^{*}} \otimes \mathscr{R}_{m} \rightarrow \wedge^{j+1} \mathscr{T}^{*} \otimes \mathscr{R}_{m}
$$


is the differential operator determined by

$$
\nabla^{\wedge}(\omega \otimes u)=d \omega \otimes u+(-1)^{j} \omega \wedge \nabla u
$$

for $\omega \in \wedge^{j} \mathscr{T}^{*}$ and $u \in \mathscr{R}_{m}$, then

$$
\nabla^{\wedge}(\omega \wedge u)=d \omega \wedge u+(-1)^{j} \omega \wedge \nabla^{\wedge} u
$$

for $\omega \in \wedge^{j} \mathscr{T}^{*}$ and $u \in \wedge \mathscr{T}^{*} \otimes \mathscr{R}_{m}$, and from (1.4) we infer that

$$
\nabla^{\wedge}=D \cdot \chi: \Lambda^{j} \mathscr{T}^{*} \otimes \mathscr{R}_{m} \rightarrow \wedge^{j+1} \mathscr{T}^{*} \otimes \mathscr{R}_{m} .
$$

Hence by the commutativity of (1.7), we see that

$$
\pi_{m-1} \nabla^{\wedge}=D: \Lambda^{j} \mathscr{T}^{*} \otimes \mathscr{R}_{m} \rightarrow \Lambda^{j+1} \mathscr{T}^{*} \otimes J_{m-1}(\mathscr{T}) .
$$

By (1.12), the symbol

$$
\sigma\left(\nabla^{\wedge}\right): T^{*} \otimes \wedge^{j} T^{*} \otimes R_{m} \rightarrow \wedge^{j+1} T^{*} \otimes R_{m}
$$

of $\nabla^{\wedge}$ sends $\alpha \otimes \omega \otimes u$ into $\alpha \wedge \omega \otimes u$, for $\alpha \in T^{*}, \omega \in \wedge^{j} T^{*}$ and $u \in R_{m}$. According to Proposition 5.1 of [8] and Theorem 2.1 of [5], the curvature of the connection $\chi$ vanishes and so the sequence

$$
0 \rightarrow \Theta \stackrel{j_{m}}{\rightarrow} \mathscr{R}_{m} \stackrel{\nabla}{\rightarrow} \mathscr{T}^{*} \otimes \mathscr{R}_{m} \stackrel{\nabla^{-}}{\rightarrow} \wedge^{2} \mathscr{T}^{*} \otimes \mathscr{R}_{m} \stackrel{\nabla^{\wedge}}{\rightarrow} \cdots \rightarrow \wedge^{n} \mathscr{T}^{*} \otimes \mathscr{R}_{m} \rightarrow 0
$$

is a complex, which by Lemma 5.3 of [8] and Proposition 2.1 of [5] is in fact an exact sequence. Moreover, by Theorem 2.2 of [8], if $U$ is a connected and simply connected open subset of $X$ and $x \in U$, the mapping

$$
\Gamma(U, \Theta) \rightarrow R_{m, x},
$$

sending $s$ into $j_{m}(s)(x)$, is an isomorphism, and so $\Theta$ is a locally constant sheaf. The sequence (1.14) is also an elliptic complex.

Henceforth, in this section we suppose that $E=T$. Consider the bracket

$$
J_{r+1}(T) \otimes J_{r+1}(T) \rightarrow J_{r}(T)
$$

defined by

$$
\left[j_{r+1}(\xi)(x), j_{r+1}(\eta)(x)\right]=j_{r}([\xi, \eta])(x)
$$

for $x \in X$ and $\xi, \eta \in \mathscr{T}_{x}$. We also assume that $R_{k}$ is a Lie equation, that is, $\left[R_{k+1}, R_{k+1}\right] \subset R_{k}$. Then we have

$$
\left[R_{m+1}, R_{m+1}\right] \subset R_{m},
$$

and the bracket

$$
R_{m} \otimes R_{m} \rightarrow R_{m},
$$

defined by

$$
[\xi, \eta]=[\chi(\xi), \chi(\eta)],
$$

for $\xi, \eta \in R_{m}$, endows $R_{m, x}$ with a structure of Lie algebra for all $x \in X$. Moreover, with respect to the Lie bracket of vector fields, $\Theta$ is a sheaf of Lie algebras, and the mapping (1.15) is an isomorphism of Lie algebras. We say that $R_{k}$ is formally transitive if $\pi_{0}: R_{k} \rightarrow T$ is surjective.

Let $B: R_{m} \otimes R_{m} \rightarrow \mathbf{R}$ be the mapping whose restriction to the fiber over $x \in X$ is the Killing form $B_{x}$ of $R_{m, x}$. 
LEMMA 1.1. If $\xi$, $\eta$ are sections of $\Theta$ over an open set $U$, then $d B\left(j_{m}(\xi), j_{m}(\eta)\right)=0$ on $U$.

Proof. We may assume that $U$ is connected and simply connected. Let $B^{\prime}$ denote the Killing form of the Lie algebra $\Gamma(U, \Theta)$. Since $(1.15)$ is an isomorphism of Lie algebras for $x \in U$, we have

$$
B^{\prime}(\xi, \eta)=B_{x}\left(j_{m}(\xi)(x), j_{m}(\eta)(x)\right)
$$

for all $\xi, \eta \in \Gamma(U, \Theta)$.

We define a morphism of vector bundles

$$
\left(\wedge^{i} T^{*} \otimes R_{m}\right) \otimes\left(\wedge^{j} T^{*} \otimes R_{m}\right) \rightarrow \wedge^{i+j} T^{*}
$$

sending $u \otimes v$ into $\langle u, v\rangle$, for $u \in \Lambda^{i} T^{*} \otimes R_{m}$ and $v \in \Lambda^{j} T^{*} \otimes R_{m}$, by setting

$$
\langle u, v\rangle=B(\xi, \eta) \alpha \wedge \beta,
$$

whenever $u=\alpha \otimes \xi$ and $v=\beta \otimes \eta$, with $\alpha \in \Lambda^{i} T^{*}, \beta \in \Lambda^{j} T^{*}$ and $\xi, \eta \in R_{m}$.

LEMMA 1.2. We have

$$
\langle\nabla \hat{u}, v\rangle+(-1)^{i}\langle u, \nabla \hat{v}\rangle=d\langle u, v\rangle
$$

for $u \in \Lambda^{i \mathscr{T}^{*} \otimes} \mathscr{R}_{m}$ and $v \in \Lambda^{j \mathscr{T}^{*}} \otimes \mathscr{R}_{m}$.

Proof. According to Lemma 1.1, the equality (1.18) holds when $u=j_{m}(\xi)$ and $v=j_{m}(\eta)$, with $\xi, \eta \in \Theta$. If (1.18) holds for $u=\xi \in \mathscr{R}_{m}$ and $v=\eta \in \mathscr{R}_{m}$, and if $f, g \in \mathcal{O}_{X}$, then by (1.12) it holds for $u=f \xi$ and $v=g \eta$. Since (1.15) is an isomorphism, the sheaf $\mathscr{R}_{m}$ is generated by $j_{m}(\Theta)$ over $\mathcal{O}_{X}$, and therefore the formula is true for all $u, v \in \mathscr{R}_{m}$. From (1.12), we see that if (1.18) holds for $u, v \in \mathscr{R}_{m}$, it holds for $u, v$ of arbitrary degree.

If $\nabla$ also denotes the connection in $R_{m}^{*} \otimes R_{m}^{*}$ induced by $\nabla$ and if we consider $B$ as a section of $R_{m}^{*} \otimes R_{m}^{*}$, then formula (1.18) holds for all $u, v \in \mathscr{R}_{m}$ if and only if $\nabla B=0$.

We denote by $H^{j}$ the cohomology of the complex

$$
\begin{aligned}
0 & \rightarrow C^{\infty}\left(R_{m}\right) \stackrel{\nabla}{\rightarrow} C^{\infty}\left(T^{*} \otimes R_{m}\right) \stackrel{\nabla^{\wedge}}{\rightarrow} C^{\infty}\left(\bigwedge^{2} T^{*} \otimes R_{m}\right) \\
& \stackrel{\nabla^{\wedge}}{\rightarrow} \cdots \rightarrow C^{\infty}\left(\Lambda^{n} T^{*} \otimes R_{m}\right) \rightarrow 0
\end{aligned}
$$

at $C^{\infty}\left(\wedge^{j} T^{*} \otimes R_{m}\right)$, and by $H_{0}^{j}$ the cohomology of the complex (1.19), with $C^{\infty}$ replaced by $C_{0}^{\infty}$, at $C_{0}^{\infty}\left(\bigwedge^{j} T^{*} \otimes R_{m}\right)$. The exact sequence (1.14) gives us canonical isomorphisms

$$
H^{j}(X, \Theta) \rightarrow H^{j}, \quad H_{0}^{j}(X, \Theta) \rightarrow H_{0}^{j},
$$

where $H_{0}^{j}(X, \Theta)$ is the $j$ th cohomology group of $X$ with compact support and values in $\Theta$.

Suppose that $X$ is oriented. Consider the mapping sending $u \otimes v$, with $u \in$ $C^{\infty}\left(\wedge^{j} T^{*} \otimes R_{m}\right)$ and $v \in C_{0}^{\infty}\left(\wedge^{n-j} T^{*} \otimes R_{m}\right)$ satisfying $\nabla \hat{u}=0$ and $\nabla \hat{v}=0$, 
onto $\int_{X}\langle u, v\rangle$; by Lemma 1.2 and Stokes' theorem, we see that this mapping induces a well-defined pairing

$$
H^{j} \otimes H_{0}^{n-j} \rightarrow \mathbf{R} .
$$

From the isomorphisms (1.20) and the pairing (1.21), we obtain a pairing

$$
H^{j}(X, \Theta) \otimes H_{0}^{n-j}(X, \Theta) \rightarrow \mathbf{R} .
$$

THEOREM 1.1. If $R_{m, x}$ is a semisimple Lie algebra for all $x \in X$, then the pairings (1.21) and (1.22) are nondegenerate.

Proof. First, we assume that $X$ is connected and simply connected; let $\omega$ be an $n$-form with compact support such that $\int_{X} \omega=1$. Since $\nabla$ is a flat connection, if $\left\{\xi_{1}, \ldots, \xi_{q}\right\}$ is a basis of $\Gamma(X, \Theta)$ and $\alpha_{j}$ denotes the cohomology class of $\omega \otimes j_{m}\left(\xi_{j}\right)$ in $H_{0}^{n}$, then $\left\{j_{m}\left(\xi_{1}\right), \ldots, j_{m}\left(\xi_{q}\right)\right\}$ is a basis of $H^{0}$ and $\left\{\alpha_{1}, \ldots, \alpha_{q}\right\}$ is a basis of $H_{0}^{n}$. Then, if $x \in X$, the pairing (1.21) sends $j_{m}\left(\xi_{i}\right) \otimes \alpha_{j}$, with $1 \leqslant i, j \leqslant q$, into

$$
\begin{aligned}
\int_{X} B\left(j_{m}\left(\xi_{i}\right), j_{m}\left(\xi_{j}\right)\right) \omega & =B\left(j_{m}\left(\xi_{i}\right)(x), j_{m}\left(\xi_{j}\right)(x)\right) \int_{X} \omega \\
& =B\left(j_{m}\left(\xi_{i}\right)(x), j_{m}\left(\xi_{j}\right)(x)\right),
\end{aligned}
$$

by Lemma 1.1. Since $B_{x}$ is nondegenerate and $\left\{j_{m}\left(\xi_{1}\right)(x), \ldots, j_{m}\left(\xi_{q}\right)(x)\right\}$ is a basis of $R_{m, x}$, the pairing (1.21) is also nondegenerate for $j=0$. Since $H^{j}=0$ for $j>0$, and $H_{0}^{j}=0$ for $j<n$, we see that (1.21) is nondegenerate for all $j \geqslant 0$. A proof due to J. Milnor of Poincaré duality for de Rham cohomology, using Mayer-Vietoris sequences for this cohomology with or without compact support and the existence of acyclic coverings, and similar to his proof of the Duality Theorem A.9 of [15, Appendix A] (see also [18, Chapter 11]), is now easily extended to the case of the cohomologies of the sequence (1.14) and gives us the nondegeneracy of (1.21) for an arbitrary oriented manifold.

Under the further assumption that $X$ is compact, we now give another proof of Theorem 1.1 using Hodge theory.

We first begin with

LEMMA 1.3. Let $\mathfrak{g}$ be a bundle of Lie algebras over $X$ and $\tau: \mathfrak{g} \rightarrow \mathfrak{g}$ a morphism of vector bundles such that $\tau_{x}$ is an involutive automorphism of $\mathrm{g}_{x}$ for all $x \in X$. Assume that $\mathfrak{g}_{x}$ is semisimple for all $x \in X$, and that $(\mathfrak{g}, \tau)$ is locally trivial, that is, for all $x_{0} \in X$ there exist a neighborhood $U$ of $x_{0}$ and an isomorphism $\Phi: U \times \mathfrak{g}_{x_{0}} \rightarrow \mathfrak{g}_{\mid U}$ of bundles of Lie algebras over $U$ such that $\tau_{x}=\Phi_{x}\left(\mathrm{id} \times \tau_{x_{0}}\right) \Phi_{x}^{-1}$. Then there exists $a$ morphism $\sigma: \mathfrak{g} \rightarrow \mathfrak{g}$ of vector bundles over $X$ such that $\sigma \tau=\tau \sigma$ and $\sigma_{x}$ is a Cartan involution of $\mathrm{g}_{x}$ for all $x \in X$.

Proof. Fix $x \in X$; let $G$ be the adjoint group of the Lie algebra $\mathfrak{g}_{x}$. According to Lemma 10.2 of [1] (see also [10, p. 156]), there exists a Cartan involution $\theta$ of $\mathfrak{g}_{x}$ commuting with $\tau_{x}$. Let $\mathfrak{g}_{x}=\mathfrak{f} \oplus \mathfrak{p}$ be the corresponding Cartan decomposition of $\mathfrak{g}_{x}$ and set $\mathfrak{h}=\left\{\xi \in \mathfrak{g}_{x} \mid \tau(\xi)=\xi\right\}$. Then according to Lemma 4 of [14] and its proof, the set of all Cartan involutions of $\mathfrak{g}_{x}$ commuting with $\tau_{x}$ is equal to

$$
\left\{\psi \theta \psi^{-1} \mid \psi=\exp (\operatorname{ad} \xi) \text {, with } \xi \in \mathfrak{p} \cap \mathfrak{h}\right\} \text {. }
$$


By [4, Chapter I, Proposition 6 and 10, p. 216], this set considered as a subset of $\operatorname{Hom}\left(\mathfrak{g}_{x}, \mathfrak{g}_{x}\right)$ is diffeomorphic to $\mathfrak{p} \cap \mathfrak{h}$. Suppose that $X$ is connected. Our condition of local triviality implies that

$$
\left\{\begin{array}{l|l}
\sigma \in \operatorname{Hom}(\mathfrak{g}, \mathfrak{g}) & \begin{array}{l}
\sigma_{a} \text { is a Cartan involution of } \mathfrak{g}_{a} \\
\text { for all } a \in X, \text { and } \sigma \tau=\tau \sigma
\end{array}
\end{array}\right\}
$$

is a locally trivial sub-bundle of $\operatorname{Hom}(\mathfrak{g}, \mathfrak{g})$ whose fiber is diffeomorphic to $\mathfrak{p} \cap \mathfrak{h}$. Therefore by Theorem 12.2 of [19], this bundle admits a differentiable section $\sigma$.

Since the mappings (1.15) are isomorphisms of Lie algebras, $R_{m}$ is a locally trivial bundle of Lie algebras. Assume that $R_{m, x}$ is a semisimple Lie algebra for all $x \in X$. Then by Lemma 1.3, with $\tau=$ id, there exists a section $\sigma$ of $R_{m}^{*} \otimes R_{m}$ over $X$ such that $\sigma_{x}$ is a Cartan involution of $R_{m, x}$ for $x \in X$. Then the mapping $B_{\sigma}: R_{m} \otimes R_{m} \rightarrow$ $\mathbf{R}$, defined by

$$
B_{\sigma}(\xi, \eta)=-B(\xi, \sigma(\eta))
$$

for $\xi, \eta \in R_{m}$, determines a symmetric positive definite form on $R_{m, x}$ for $x \in X$. Fix a Riemannian metric $g$ on $X$ and consider the scalar product ( , ) on $\wedge T^{*} \otimes R_{m}$ induced by the scalar product on $\wedge T^{*}$ determined by $g$ and the scalar product $B_{\sigma}$ on $R_{m}$. Suppose that $X$ is oriented and let $\Omega$ be the corresponding volume form on $X$. Let

$$
*: \wedge^{j} T^{*} \otimes R_{m} \rightarrow \wedge^{n-j} T^{*} \otimes R_{m}
$$

be the morphism of vector bundles defined in terms of the Hodge $*$-isomorphism by

$$
*(\alpha \otimes \xi)=-(* \alpha) \otimes \sigma(\xi)
$$

for $\alpha \in \wedge^{j} T^{*}$ and $\xi \in R_{m}$. Then, since $\sigma$ is an involution,

$$
* * u=(-1)^{n j+j} u
$$

for $u \in \wedge^{j} T^{*} \otimes R_{m}$. Moreover, for $u, v \in \wedge^{j} T^{*} \otimes R_{m}$, we have

$$
\langle u, * v\rangle=(u, v) \Omega \text {. }
$$

Indeed, if $u=\alpha \otimes \xi$ and $v=\beta \otimes \eta$, with $\alpha, \beta \in \wedge^{j} T^{*}$ and $u, v \in R_{m}$, we see that

$$
\begin{aligned}
\langle u, * v\rangle & =-\langle\alpha \otimes \xi, * \beta \otimes \sigma(\eta)\rangle=-B(\xi, \sigma(\eta)) \alpha \wedge * \beta \\
& =B_{\sigma}(\xi, \eta) \alpha \wedge * \beta=(u, v) \Omega .
\end{aligned}
$$

From formulas (1.24), (1.25), Lemma 1.2 and Stokes' theorem, it follows that the formal adjoint

$$
\nabla^{\wedge *}: \wedge^{j+1} \mathscr{T}^{*} \otimes \mathscr{R}_{m} \rightarrow \wedge^{j} \mathscr{T}^{*} \otimes \mathscr{R}_{m}
$$

of $\nabla^{\wedge}$ (with respect to $\Omega$ and the scalar product (, ) on $\wedge T^{*} \otimes R_{m}$ ) is equal to

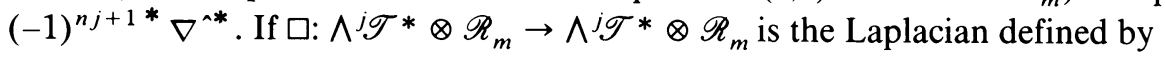

then

$$
\square=\nabla^{\wedge} \nabla^{\wedge}+\nabla^{\wedge} \nabla^{\wedge}
$$

$$
* \square=\square *
$$

(see [7, Proposition 14.1]). Moreover, if $X$ is compact, since (1.14) (with $\Theta$ omitted) is an elliptic complex, according to Hodge theory the harmonic space

$$
\mathbf{H}^{j}=\left\{u \in C^{\infty}\left(\wedge^{j} T^{*} \otimes R_{m}\right) \mid \square u=0\right\}
$$


is finite-dimensional and the mapping $\mathbf{H}^{j} \rightarrow H^{j}$, sending $u$ into the cohomology class of $u$ in $H^{j}$, is an isomorphism. By (1.26), the mapping (1.23) induces an isomorphism

$$
*: \mathbf{H}^{j} \rightarrow \mathbf{H}^{n-j} .
$$

From these results and (1.25), we deduce that (1.21) is a nondegenerate pairing (see for example the proof of Theorem 14.1 of [7]).

2. Filtrations and spectral sequences. In this section, we shall consider modules over a ring $R$. Let $A$ be a filtered complex (over $R$ ) endowed with a filtration

$$
\cdots \subset F^{p+1}(A) \subset F^{p}(A) \subset \cdots
$$

by submodules of $A$, with $p \in \mathbf{Z}$ and $A=\cup_{p \in \mathbf{Z}} F^{p}(A)$; moreover $A=\bigoplus_{j \in \mathbf{Z}} A^{j}$ is graded and, if $F^{p . j}(A)=A^{j} \cap F^{p}(A)$, we have

$$
F^{p}(A)=\bigoplus_{j \in \mathbf{Z}} F^{p \cdot j}(A) .
$$

The differential $d$ of the complex $A$ satisfies

$$
d F^{p, j}(A) \subset F^{p, j+1}(A) .
$$

We consider the spectral sequence of this filtered complex; we set

$$
\begin{gathered}
Z_{r}^{p, j}=\left\{a \in F^{p . j}(A) \mid d a \in F^{p+r, j+1}(A)\right\}, \\
E_{r}^{p . j}=Z_{r}^{p . j} /\left(Z_{r-1}^{p+1 . j}+d Z_{r-1}^{p-r+1, j-1}\right) .
\end{gathered}
$$

We have

$$
E_{0}^{p, j}=F^{p, j}(A) / F^{p+1, j}(A) .
$$

The differential $d$ induces a morphism $d_{r}: E_{r}^{p . j} \rightarrow E_{r}^{p+r, j+1}$. Then $E_{r+1}^{p, j}$ is canonically isomorphic to the cohomology of the sequence

$$
E_{r}^{p-r, j-1} \stackrel{d_{r}}{\rightarrow} E_{r}^{p, j} \stackrel{d_{r}}{\rightarrow} E_{r}^{p+r . j+1}
$$

at $E_{r}^{p . j}$. Thus if $E_{r}^{p+r . j+1}=0$, there is a natural epimorphism $E_{r}^{p . j} \rightarrow E_{r+1}^{p, j}$, and if $E_{r}^{p-r . j-1}=0$, there is a natural monomorphism $E_{r+1}^{p . j} \rightarrow E_{r}^{p . j}$. Let $r<s$; by composing such mappings, from this remark we obtain a natural epimorphism $E_{r}^{p, j} \rightarrow E_{s}^{p, j}$ if $E_{r}^{q . j+1}=0$ for $p+r \leqslant q<p+s$, and a natural monomorphism $E_{s}^{p, j} \rightarrow E_{r}^{p, j}$ if $E_{r}^{q . j-1}=0$ for $p-s<q \leqslant p-r$; if both assumptions hold, these mappings are reciprocal isomorphisms.

Let $r \geqslant 1$; assume that

$$
\begin{gathered}
E_{0}^{p \cdot j+1}=0, \quad E_{1}^{q . j+1}=0, \\
E_{0}^{p+r . j}=0, \quad E_{1}^{q . j}=0
\end{gathered}
$$

for $p<q<p+r$. Then we have a natural epimorphism $\alpha: E_{\delta}^{p, j} \rightarrow E_{r}^{p, j}$ and a natural monomorphism $\beta: E_{r}^{p+r . j+1} \rightarrow E_{0}^{p+r . j+1}$ and so we may consider the morphism

$$
d_{0, r}: E_{0}^{p, j} \rightarrow E_{0}^{p+r, j+1}
$$


equal to the composition

$$
E_{0}^{p, j} \stackrel{\alpha}{\rightarrow} E_{r}^{p, j} \stackrel{d_{r}}{\rightarrow} E_{r}^{p+r, j+1} \stackrel{\beta}{\rightarrow} E_{0}^{p+r, j+1} .
$$

This operator $d_{0, r}$ is defined under stronger assumptions in [3, Chapter XV, §5]. If (2.1) holds for $p<q<p+r$, then

$$
F^{p, j}(A)=Z_{r}^{p, j}+F^{p+1, j}(A) ;
$$

if $u \in F^{p, j}(A)$ is written as

$$
u=v+w,
$$

where $v \in Z_{r}^{p, j}$ and $w \in F^{p+1, j}(A)$, then it is easily verified that the image under $\alpha$ of the class $[u]$ of $u$ in $E^{p, j}$ is equal to the class of $v$ in $E_{r}^{p, j}$. On the other hand, if (2.2) holds for $p<q<p+r$, we have

$$
d Z_{r-1}^{p+1, j} \subset F^{p+r+1, j+1}(A) ;
$$

if $u^{\prime} \in Z_{r}^{p+r, j+1}$, the image under $\beta$ of the class of $u^{\prime}$ in $E_{r}^{p+r, j+1}$ is equal to the class of $u^{\prime}$ in $E_{b^{+r, j+1}}$. Hence if (2.1) and (2.2) hold for $p<q<p+r$, and if $u \in F^{p, j}(A)$ admits the decomposition (2.3), then $d_{0, r}[u]$ is equal to the class of $d v$ in $E_{\delta^{+r, j+1}}$. For $r=0$, we set $d_{0,0}=d_{0}$.

Let $r, s$ be integers $\geqslant 0$. If $r \geqslant 1$, assume (2.1) and (2.2) hold for $p<q<p+r$; if $s \geqslant 1$, suppose that

$$
\begin{array}{cc}
E_{0}^{p-s, j}=0, & E_{1}^{q, j}=0, \\
E_{0}^{p^{j-1}}=0, & E_{1}^{p, j-1}=0
\end{array}
$$

for $p-s<q<p$. Under these assumptions, we have the sequence

$$
E_{0}^{p-s, j-1} \stackrel{d_{0 . s}}{\rightarrow} E_{0}^{p, j} \stackrel{d_{0 . r}}{\rightarrow} E_{0}^{p+r, j+1},
$$

which is easily seen to be a complex.

The proof of the following proposition is similar to that of Proposition 5.9 of [3, Chapter XV].

Proposition 2.1. Suppose that one of the following conditions hold:

(a) $r=s$;

(b) $s<r$ and

$$
E_{1}^{q . j-1}=0 \quad \text { for } p-r \leqslant q<p-s
$$

(c) $s>r$ and

$$
E_{1}^{q, j+1}=0 \text { for } p+r<q \leqslant p+s .
$$

Then, if $t=\sup (r, s)+1$, the cohomology of the complex (2.6) is isomorphic to $E_{t}^{p, j}$. 
Proof. First, if $r=s$, we have a commutative diagram

$$
\begin{array}{lllll}
E_{0}^{p-r, j-1} & \stackrel{d_{0, r}}{\rightarrow} & E_{0}^{p, j} & \stackrel{d_{0, r}}{\rightarrow} & E_{0}^{p+r, j+1} \\
\downarrow \alpha & & \downarrow \beta & & \uparrow \gamma \\
E_{r}^{p-r, j-1} & \stackrel{d_{r}}{\rightarrow} & E_{r}^{p, j} & \stackrel{d_{r}}{\rightarrow} & E_{r}^{p+r, j+1}
\end{array}
$$

where $\alpha$ is an epimorphism, $\beta$ is an isomorphism and $\gamma$ is a monomorphism. Hence the cohomology of (2.6) is isomorphic to the cohomology of the bottom row of this diagram, that is, to $E_{r+1}^{p, j}$.

Next, suppose that (b) holds. In this case, we have a commutative diagram

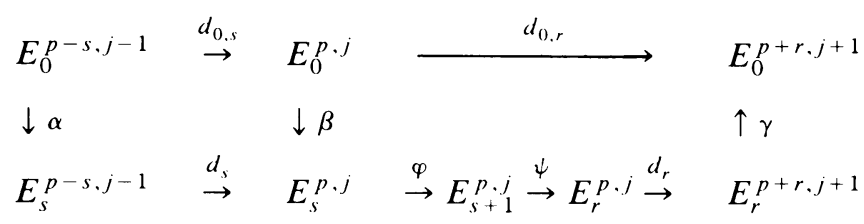

where $\alpha, \varphi$ and $\psi$ are epimorphisms, $\beta$ is an isomorphism and $\gamma$ is a monomorphism. The hypothesis (2.7) implies that $\psi$ is an isomorphism and that $E_{r}^{p-r, j+1}=0$; from this last fact, we obtain an exact sequence

$$
0 \rightarrow E_{r+1}^{p, j} \stackrel{\chi}{\rightarrow} E_{r}^{p, j} \stackrel{d_{r}}{\rightarrow} E_{r}^{p+r, j+1} .
$$

From (2.1), we deduce that $E_{s}^{p+s . j+1}=0$; hence the sequence

$$
E_{s}^{p-s, j-1} \stackrel{d_{s}}{\rightarrow} E_{s}^{p, j} \stackrel{\varphi}{\rightarrow} E_{s+1}^{p, j} \rightarrow 0
$$

is exact. We now easily see that the cohomology of the complex

$$
E_{s}^{p-s, j-1} \stackrel{d_{s}}{\rightarrow} E_{s}^{p, j} \stackrel{d_{r} \cdot \psi \cdot \varphi}{\rightarrow} E_{r}^{p+r, j+1}
$$

is isomorphic to $E_{r+1}^{p, j}$, and therefore so is the cohomology of (2.6).

Finally, assume that (c) holds. We then have a commutative diagram

$$
\begin{array}{lllll}
E_{0}^{p-s, j-1} & \stackrel{d_{0, s}}{\longrightarrow} & E_{0}^{p, j} & \stackrel{d_{0, r}}{\rightarrow} & E_{0}^{p+r, j+1} \\
\downarrow \alpha & & \downarrow \beta & & \uparrow \gamma \\
E_{s}^{p-s, j-1} & \stackrel{d_{s}}{\rightarrow} E_{s}^{p, j} \stackrel{\psi}{\rightarrow} E_{r+1}^{p, j} \stackrel{\chi}{\rightarrow} & E_{r}^{p, j} & \stackrel{d_{r}}{\rightarrow} & E_{r}^{p+r, j+1}
\end{array}
$$

where $\alpha$ is an epimorphism, $\beta$ is an isomorphism and $\psi, \chi$ and $\gamma$ are monomorphisms. The hypothesis (2.8) implies that $\psi$ is an isomorphism and that $E_{s}^{p+s, j+1}=0$; from this last fact, we obtain the exact sequence (2.10). From (2.5), we deduce that $E_{r}^{p-r . j-1}=0$, and so the sequence (2.9) is exact. We now easily see that the cohomology of the complex

$$
E_{s}^{p-s, j-1} \stackrel{\chi \cdot \psi \cdot d_{s}}{\rightarrow} E_{r}^{p, j} \stackrel{d_{r}}{\rightarrow} E_{r}^{p+r, j+1}
$$

is isomorphic to $E_{s+1}^{p, j}$, and therefore so is the cohomology of (2.6). 
We say that the filtration is regular if, for each $j$, there exists an integer $p_{j}$ such that $F^{p}(A) \cap A^{j}=0$ for $p>p_{j}$. We denote by $H^{j}(A)$ the cohomology at $A^{j}$ of the complex

$$
A^{j-1} \stackrel{d}{\rightarrow} A^{j} \stackrel{d}{\rightarrow} A^{j+1}
$$

If there exists $t \geqslant 0$ such that $E_{t}^{q, j}=0$ for $q<p$, then any element $u$ of $A^{j}$ satisfying $d u=0$ can be written as $u=v+d w$ for some $v \in F^{p, j}(A)$ and $w \in A^{j-1}$.

The following result is given by Proposition 5.6 of [3, Chapter XV].

Proposition 2.2. Let $t \geqslant 0$. Assume that the filtration is regular and that

$$
\begin{array}{ll}
E_{t}^{q, j-1}=0 & \text { for } q \leqslant p-t, \\
E_{t}^{q, j}=0 & \text { for } q \neq p, \\
E_{t}^{p, j+1}=0 & \text { for } q \geqslant p+t .
\end{array}
$$

Then we have a natural isomorphism $H^{j}(A) \rightarrow E_{t}^{p, j}$.

If the hypotheses of Proposition 2.2 hold, and if $u \in A^{j}$ satisfies $d u=0$, then because of (2.11) we may write $u=v+d w$ for some $v \in F^{p, j}(A)$ and $w \in A^{j-1}$; the mapping $H^{j}(A) \rightarrow E_{t}^{p, j}$ of Proposition 2.2 sends the class of $u$ in $H^{j}(A)$ into the class of $v \in Z_{t}^{p, j}$ in $E_{t}^{p, j}$.

From Propositions 2.1 and 2.2, we deduce

Proposition 2.3. Let $t=\sup (r, s)$. Suppose that the assumptions of Proposition 2.2 and that one of conditions (a), (b), (c) of Proposition 2.1 hold. Then the cohomology of the complex (2.6) is naturally isomorphic to $H^{j}(A)$.

In fact, under the assumptions of Proposition 2.3, we have a natural isomorphism from $H^{j}(A)$ to the cohomology of (2.6) which can be described as follows. If $u \in A^{j}$ satisfies $d u=0$, then we may write $u=v+d w$ for some $v \in F^{p, j}(A)$ and $w \in A^{j-1}$; this isomorphism sends the class of $u$ in $H^{j}(A)$ into the class of $v \in Z_{0}^{p, j}$ in $E_{0}{ }^{, j}$.

For the remainder of this section, suppose that $K$ is a graded filtered module (over $R$ ), with a filtration

$$
K=K^{0} \supset K^{1} \supset \cdots \supset K^{p} \supset K^{p+1} \supset \cdots
$$

and a gradation $K=\bigoplus_{j \in \mathbf{Z}} A^{j}$; if $K^{p, j}=K^{p} \cap A^{j}$, we suppose that

$$
K^{p}=\bigoplus_{p \in \mathbf{Z}} K^{p, j}
$$

We set $K^{p}=K$ and $K^{p, j}=A^{j}$ for $p<0$. Assume that $K$ is a complex and that its differential $d$ satisfies

$$
d K^{p, j} \subset K^{p-1, j+1} .
$$

By (2.12), we obtain a new filtration

$$
\cdots \subset F^{p+1}(K) \subset F^{p}(K) \subset \cdots
$$

on $K$ by setting

$$
F^{2 p}(K)=\left\{u \in K^{p} \mid d u \in K^{p}\right\}, \quad F^{2 p+1}(K)=K^{p+1}+d K^{p+1}
$$


for $p \geqslant 0$, and $F^{p}(K)=K$ for $p<0$. We write $F^{p . j}(K)=F^{p}(K) \cap K^{j}$; then

$$
\begin{gathered}
F^{2 p, j}(K)=\left\{u \in K^{p . j} \mid d u \in K^{p, j+1}\right\}, \\
F^{2 p+1, j}(K)=K^{p+1, j}+d K^{p+1, j-1}
\end{gathered}
$$

and $F^{p}(K)=\oplus_{j \in \mathbf{Z}} F^{p, j}(K)$ for $p \geqslant 0$. Clearly,

$$
d F^{p, j}(K) \subset F^{p, j+1}(K)
$$

for all $p$, and so $K$ endowed with the filtration (2.13) is a filtered complex. We now consider its spectral sequence. For simplicity, we write $F^{p}=F^{p}(K)$. From the definition of this filtration, it follows directly that

$$
Z_{1}^{2 p-1}=F^{2 p}+d F^{2 p-1}
$$

for $p \geqslant 1$. Therefore, we have $E_{1}^{2 p-1}=0$ for $p \geqslant 1$. Hence $H^{j}\left(F^{2 p-1} / F^{2 p}\right)=0$ for $p \geqslant 1$, and so we obtain

LEMMA 2.1. For $p \geqslant 1$, we have $E_{1}^{2 p-1}=0$ and the mapping $H^{j}\left(F^{2 p}\right) \rightarrow H^{j}\left(F^{2 p-1}\right)$ is an isomorphism.

3. Filtrations and resolutions. Let $R_{m} \subset J_{m}(E)$ be a formally integrable differential equation of order $m$ on a vector bundle $E$. Assume that $g_{m+1}=0$; we consider the exact sequence (1.14). Suppose also that $\pi_{0}: R_{m} \rightarrow E$ is surjective and that the kernel $R_{m}^{l}$ of $\pi_{l}: R_{m} \rightarrow J_{l}(E)$ is a vector bundle for all $0 \leqslant l \leqslant m$; we thus obtain a filtration

$$
\cdots \subset R_{m}^{l+1} \subset R_{m}^{l} \subset \cdots \subset R_{m}^{0} \subset R_{m}^{-1}=R_{m},
$$

where $R_{m}^{l}=0$ for $l \geqslant m$, by sub-bundles. For $0 \leqslant l \leqslant m$, let $g_{l}$ be the sub-bundle of $S^{\prime} T^{*} \otimes E$ defined by the exact sequence

$$
0 \rightarrow g_{l} \stackrel{\varepsilon}{\rightarrow} \pi_{l} R_{m} \stackrel{\pi_{l-1}}{\rightarrow} \pi_{l-1} R_{m} \rightarrow 0,
$$

where $\pi_{-1}=0$. We identify $g_{l}$ with its image in $J_{l}(E)$ under the mapping $\varepsilon$. We have $g_{m}=R_{m}^{m-1}$ and $g_{0}=E$. Since $R_{m}$ is formally integrable, from the commutativity of diagram (1.7) we see that

$$
D\left(\wedge^{j \mathscr{T} *} \otimes \mathscr{R}_{1+1}\right) \subset \wedge^{j+1} \mathscr{T}^{*} \otimes \mathscr{R}_{l}
$$

for $0 \leqslant l \leqslant m-1$. We therefore obtain a complex

$$
\begin{aligned}
0 & \rightarrow g_{l} \stackrel{\delta}{\rightarrow} T^{*} \otimes g_{l-1} \stackrel{\delta}{\rightarrow} \wedge^{2} T^{*} \otimes g_{l-2} \\
& \stackrel{\delta}{\rightarrow} \cdots \rightarrow \wedge^{\prime-1} T^{*} \otimes g_{1} \stackrel{\delta}{\rightarrow} \wedge^{\prime} T^{*} \otimes g_{0} \rightarrow 0
\end{aligned}
$$

for all $l \geqslant 0$; the Spencer cohomology $H^{l-j . j}\left(R_{m}\right)$ of $R_{m}$, with $l-j \geqslant 0$, is the cohomology of (3.1) at $\wedge^{j} T^{*} \otimes g_{l-j}$. We have

$$
\begin{array}{ll}
H^{0.0}\left(R_{m}\right)=E, \quad H^{l .0}\left(R_{m}\right)=0 & \text { for } l>0, \\
H^{m+l . j}\left(R_{m}\right)=H^{m+l . j}\left(g_{m}\right)=0 & \text { for } l>0,
\end{array}
$$

and

$$
H^{m . j}\left(R_{m}\right)=H^{m, j}\left(g_{m}\right)=\left\{u \in \wedge^{j} T^{*} \otimes g_{m} \mid \delta u=0\right\},
$$


with $H^{m, 1}\left(g_{m}\right)=0$. We suppose that the mapping

$$
\delta: \wedge^{j} T^{*} \otimes g_{l} \rightarrow \wedge^{j+1} T^{*} \otimes g_{l-1},
$$

whose kernel we denote by $M_{l}^{j}$, is of constant rank for all $j, l \geqslant 0$, where $g_{-1}=0$; then $H^{l, j}\left(R_{m}\right)$ is a vector bundle for all $j, l \geqslant 0$. For $p \geqslant 0$, let

$$
\gamma_{2 p}: \wedge^{j} T^{*} \otimes g_{p} \rightarrow \frac{\wedge^{j} T^{*} \otimes g_{p}}{M_{p}^{j}}, \quad \gamma_{2 p+1}: M_{p}^{j} \rightarrow H^{p, j}\left(R_{m}\right)
$$

be the natural projections.

We define a filtration

$$
\cdots \subset C^{p+1} \subset C^{p} \subset \cdots \subset C^{0}=\wedge T^{*} \otimes R_{m}
$$

of $\wedge T^{*} \otimes R_{m}$ by sub-bundles $C^{p}$ defined as follows. For $0 \leqslant p \leqslant m$, let $C^{2 p, j}$ be the sub-bundle of $\wedge^{j} T^{*} \otimes R_{m}$ determined by the exact sequence

$$
0 \rightarrow C^{2 p, j} \rightarrow \wedge^{j} T^{*} \otimes R_{m}^{p-1} \stackrel{\gamma_{2 p} \cdot \pi_{p}}{\rightarrow} \frac{\bigwedge^{j} T^{*} \otimes g_{p}}{M_{p}^{j}} \rightarrow 0
$$

and let $C^{2 p+1, j}$ be the sub-bundle of $C^{2 p, j}$ defined by the exact sequence

$$
0 \rightarrow C^{2 p+1, j} \rightarrow C^{2 p, j} \stackrel{\gamma_{2 p+1} \cdot \pi_{p}}{\rightarrow} H^{p, j}\left(R_{m}\right) \rightarrow 0 .
$$

Then we have

$$
\wedge^{j} T^{*} \otimes R_{m}^{p} \subset C^{2 p+1, j} \subset C^{2 p, j} \subset \Lambda^{j} T^{*} \otimes R_{m}^{p-1} ;
$$

if $\varpi: C^{2 p, j} \rightarrow C^{2 p, j} / C^{2 p+1, j}$ is the natural projection, the mapping $\rho_{p}=\gamma_{2 p+1} \cdot \pi_{p}$ induces an isomorphism

$$
\mu: C^{2 p, j} / C^{2 p+1, j} \rightarrow H^{p, j}\left(R_{m}\right)
$$

such that the diagram

$$
\begin{array}{ccc}
C^{2 p, j} & \stackrel{\varpi}{\rightarrow} & C^{2 p, j} / C^{2 p+1, j} \\
& \rho_{p} \searrow & \downarrow \mu \\
& & H^{p, j}\left(R_{m}\right)
\end{array}
$$

commutes. In particular, $C^{2 m, j}=H^{m, j}\left(g_{m}\right)$ and $C^{2 m+1, j}=0$. We set $C^{p, j}=0$ for $p \geqslant 2 m+2$ and $C^{p}=\bigoplus_{j \in \mathbf{Z}} C^{p, j}$ for $p \geqslant 0$.

From (1.13) and the commutativity of (1.7), we deduce the commutativity of.

$$
\begin{array}{ccc}
\bigwedge^{j \mathscr{T}^{*} \otimes \mathscr{R}_{m}^{p-1}} & \stackrel{\nabla^{-}}{\rightarrow} & \Lambda^{j+1} \mathscr{T}^{*} \otimes \mathscr{R}_{m} \\
\downarrow \pi_{p} & & \downarrow \pi_{p-1} \\
\wedge^{j} \mathscr{T}^{*} \otimes g_{p} & \stackrel{-\delta}{\rightarrow} & \wedge^{j+1} \mathscr{T}^{*} \otimes \mathscr{R}_{p-1}
\end{array}
$$

for $0 \leqslant p \leqslant m$, and hence that

$$
\nabla^{\wedge}\left(\wedge^{j} \mathscr{T}^{*} \otimes \mathscr{R}_{m}^{p}\right) \subset \Lambda^{j+1} \mathscr{T}^{*} \otimes \mathscr{R}_{m}^{p-1}
$$


From the filtration

$$
\wedge \mathscr{T}^{*} \otimes \mathscr{R}_{m}=K^{0} \supset K^{1} \supset \cdots \supset K^{p} \supset K^{p+1} \supset \cdots
$$

of $\wedge \mathscr{T}^{*} \otimes \mathscr{R}_{m}$ defined by

$$
\begin{gathered}
K^{p, j}=\wedge^{j \mathscr{T}^{*} \otimes \mathscr{R}_{m}^{p-1}} \text { for } 0 \leqslant p \leqslant m, \\
K^{p, j}=0 \text { for } p \geqslant m+1, \text { and } K^{p}=\oplus_{j \in \mathbf{Z}} K^{p, j} \text { for } p \geqslant 0,
\end{gathered}
$$

as we have (2.12), with $d=\nabla^{\wedge}$, according to (2.14), we obtain a new filtration

$$
\cdots \subset F^{p+1} \subset F^{p} \subset \cdots
$$

on $\wedge \mathscr{T}^{*} \otimes \mathscr{R}_{m}$ by setting

$$
\begin{gathered}
F^{0, j}=\wedge^{j} \mathscr{T}^{*} \otimes \mathscr{R}_{m}, \\
F^{2 p, j}=\left\{u \in \wedge^{j} \mathscr{T}^{*} \otimes \mathscr{R}_{m}^{p-1} \mid \nabla^{\wedge} u \in \Lambda^{j+1} \mathscr{T}^{*} \otimes \mathscr{R}_{m}^{p-1}\right\}, \\
F^{2 p+1, j}=\wedge^{j} \mathscr{T}^{*} \otimes \mathscr{R}_{m}^{p}+\nabla^{\wedge}\left(\wedge^{j-1} \mathscr{T}^{*} \otimes \mathscr{R}_{m}^{p}\right)
\end{gathered}
$$

for $0 \leqslant p \leqslant m, F^{p, j}=0$ for $p \geqslant 2 m+1$, and $F^{p}=\oplus_{j \in \mathbf{Z}} F^{p . j}$.

Let $\Phi$ be a paracompactifying family of supports on $X$. If $\mathscr{A}$ is a sheaf over $X$, we denote by $\Gamma_{\Phi}(\mathscr{A})$ the space of sections of $\mathscr{A}$ over $X$ whose supports belong to the family $\Phi$, and by $H_{\Phi}(X, \mathscr{A})$ the $j$ th cohomology group of $X$ with values in $\mathscr{A}$ and supports in $\Phi$.

We consider the filtration $\left\{\Gamma_{\Phi}\left(K^{p}\right)\right\}$ of the complex $\Gamma_{\Phi}\left(\wedge \mathscr{T}^{*} \otimes \mathscr{R}_{m}\right)$. Using the commutativity of (3.5), since the mappings (3.2) are of constant rank, we easily see that

$$
\begin{gathered}
F^{p, j}=\mathscr{C}^{p, j}, \\
\Gamma_{\Phi}\left(\mathscr{C}^{2 p, j}\right)=\Gamma_{\Phi}\left(K^{p, j}\right)+\nabla^{\wedge} \Gamma_{\Phi}\left(K^{p, j-1}\right), \\
\Gamma_{\Phi}\left(\mathscr{C}^{2 p+1 . j}\right)=\left\{u \in \Gamma_{\Phi}\left(K^{p . j}\right) \mid \nabla^{\wedge} u \in \Gamma_{\Phi}\left(K^{p . j+1}\right)\right\}
\end{gathered}
$$

for all $p, j \geqslant 0$. Thus $\left\{\Gamma_{\Phi}\left(\mathscr{C}^{p}\right)\right\}$ is the filtration obtained from $\left\{\Gamma_{\Phi}\left(K^{p}\right)\right\}$ using (2.14); moreover, according to (2.15) we have $\nabla^{\wedge}\left(\mathscr{C}^{p, j}\right) \subset \mathscr{C}^{p, j+1}$. Therefore, we may consider on one hand the spectral sequence of $\wedge \mathscr{T}^{*} \otimes \mathscr{R}_{m}$ with the filtration $\left\{\mathscr{C}^{p}\right\}$ and the differential $\nabla^{\wedge}$ and on the other hand the spectral sequence of $\Gamma_{\Phi}\left(\wedge \mathscr{T}^{*} \otimes \mathscr{R}_{m}\right)$ with the filtration $\left\{\Gamma_{\Phi}\left(\mathscr{C}^{p}\right)\right\}$ and the differential $\nabla^{\wedge}$. We denote by $\mathscr{H}^{j}\left(\mathscr{C}^{p}\right)$ the cohomology of the complex

$$
\mathscr{C}^{p, j-1} \stackrel{\nabla^{-}}{\rightarrow} \mathscr{C}^{p, j} \stackrel{\nabla^{-}}{\rightarrow} \mathscr{C}^{p, j+1} .
$$

According to Lemma 2.1 , the canonical mappings $\mathscr{H}^{j}\left(\mathscr{C}^{2 p}\right) \rightarrow \mathscr{H}^{j}\left(\mathscr{C}^{2 p-1}\right)$ are isomorphisms. Moreover, we have

LemMa 3.1. Let $j>0$; if $H^{q . j}\left(R_{m}\right)=0$ and $H^{q . j-1}\left(R_{m}\right)=0$ for $0 \leqslant q<p$, then $\mathscr{H}^{j}\left(\mathscr{C}^{2 p}\right)=0$.

Proof. Our hypothesis implies that for the spectral sequence of $\wedge \mathscr{T}^{*} \otimes \mathscr{R}_{m}$, we have $E_{1}^{q, j}=0$ and $E_{1}^{q, j-1}=0$ for $0 \leqslant q<2 p$. Therefore the canonical mappings $\mathscr{H}^{j}\left(\mathscr{C}^{q+1}\right) \rightarrow \mathscr{H}^{j}\left(\mathscr{C}^{q}\right)$ are isomorphisms for $0 \leqslant q<2 p$. From the exactness of (1.14), we obtain the desired result. 
We now consider the operator $d_{r}$ of the spectral sequence of the complex $\wedge \mathscr{T}^{*} \otimes \mathscr{R}_{m}$ with the filtration $\left\{\mathscr{C}^{p}\right\}$ and the operators $d_{0, r}$ of $\S 2$ whenever they are defined. The diagram

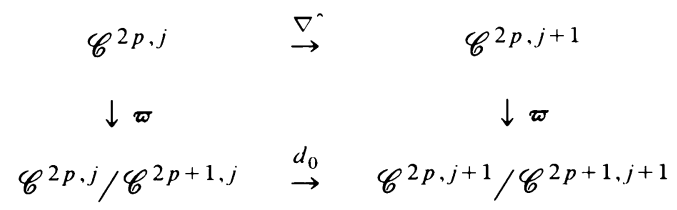

commutes; as in $\S 2$, we set $d_{0,0}=d_{0}$. As we have seen in $\S 1$, the symbol

$$
\sigma\left(\nabla^{\wedge}\right): T^{*} \otimes \wedge^{j} T^{*} \otimes R_{m} \rightarrow \wedge^{j+1} T^{*} \otimes R_{m}
$$

is induced by the multiplication of $\wedge T^{*}$. This mapping $\sigma\left(\nabla^{\wedge}\right)$ induces by restriction a morphism $T^{*} \otimes C^{2 p, j} \rightarrow C^{2 p, j+1}$, which in turn, by passage to the quotient, determines a mapping

$$
T^{*} \otimes C^{2 p, j} / C^{2 p+1, j} \rightarrow C^{2 p, j+1} / C^{2 p+1, j+1}
$$

which is equal to $\sigma\left(d_{0}\right)$.

Let $p \geqslant 0$ and $r \geqslant 1$. Assume that

$$
\begin{array}{ll}
H^{q, j+1}\left(R_{m}\right)=0 & \text { for } p \leqslant q<p+r, \\
H^{q, j}\left(R_{m}\right)=0 & \text { for } p<q \leqslant p+r .
\end{array}
$$

By Lemma 2.1 and because of the isomorphisms (3.4), the conditions (2.1) and (2.2), with $p$ replaced by $2 p$ and $r$ by $2 r$, are verified. Therefore, according to $\S 2$, we have a mapping

$$
d_{0,2 r}: \mathscr{C}^{2 p, j} / \mathscr{C}^{2 p+1, j} \rightarrow \mathscr{C}^{2(p+r), j+1} / \mathscr{C}^{2(p+r)+1, j+1} .
$$

Because of (3.8), for $0 \leqslant s<r$ the mapping

$$
\delta \cdot \pi_{p+s+1}: \bigwedge^{j} T^{*} \otimes R_{m}^{p+s} \rightarrow M_{p+s}^{j+1}
$$

is surjective. Let $\nu_{s}: M_{p+s}^{j+1} \rightarrow \wedge^{j} T^{*} \otimes R_{m}^{p+s}$ be a morphism of vector bundles such that $\delta \pi_{p+s+1} \nu_{s}=$ id. Let $v \in \mathscr{C}^{2(p+s), j+1}$; then the element $v+\nabla \hat{\nu}_{s} \pi_{p+s} v$ of $\wedge^{j+1} \mathscr{T}^{*} \otimes \mathscr{R}_{m}$ is well-defined. According to the commutativity of (3.5), we have

$$
\pi_{p+s}\left(v+\nabla \hat{\nu}_{s} \pi_{p+s} v\right)=\pi_{p+s} v-\delta \pi_{p+s+1} \nu_{s} \pi_{p+s} v=0,
$$

and so

$$
v+\nabla \hat{\nu}_{s} \pi_{p+s} v \in \Lambda^{j+1} \mathscr{T}^{*} \otimes \mathscr{R}_{m}^{p+s} .
$$

Let $u \in \mathscr{C}^{2 p, j}$ satisfy $\nabla \hat{u} \in \mathscr{C}^{2 p+2 s, j+1}$ with $0 \leqslant s<r$. Then

$$
Q_{s} u=u+\nu_{s} \pi_{p+s} \nabla \hat{u}
$$

is a well-defined element of $\wedge^{j} \mathscr{T}^{*} \otimes \mathscr{R}_{m}$, where $\nu_{s} \pi_{p+s} \nabla \hat{u}$ belongs to $\mathscr{C}^{2(p+s)+1, j}$; moreover, according to (3.9), with $v=\nabla^{\hat{u}}$, we see that $\nabla^{\wedge} Q_{s} u \in \wedge^{j+1} \mathscr{T}^{*} \otimes \mathscr{R}_{m}^{p+s}$, and hence that

$$
\nabla^{\wedge} Q_{s} u \in \mathscr{C}^{2(p+s+1), j+1}
$$


Therefore, if $u$ is an arbitrary element of $\mathscr{C}^{2 p, j}$, we obtain a well-defined element $Q_{r-1} \cdot \cdots \cdot Q_{1} \cdot Q_{0} u$ of $\mathscr{C}^{2 p, j}$, with

$$
Q u=\nabla^{\wedge} Q_{r-1} \cdot \cdots \cdot Q_{1} \cdot Q_{0} u \in \mathscr{C}^{2(p+r), j+1}
$$

and

$$
u-Q_{r-1} \cdot \cdots \cdot Q_{1} \cdot Q_{0} u \in \mathscr{C}^{2 p+1, j} .
$$

From the construction of the mapping $d_{0.2 r}$ given in $\S 2$, we see that the diagram

$$
\begin{array}{ccc}
\mathscr{C}^{2 p, j} & \stackrel{Q}{\rightarrow} & \mathscr{C}^{2(p+r), j+1} \\
\downarrow \varpi & & \downarrow \varpi \\
\mathscr{C}^{2 p, j} / \mathscr{C}^{2 p+1, j} & \stackrel{d_{0.2 r}}{\rightarrow} & \mathscr{C}^{2(p+r), j+1} / \mathscr{C}^{2(p+r)+1, j+1}
\end{array}
$$

commutes; we now see that $Q$ and $d_{0,2 r}$ are differential operators of order $r+1$.

If $\alpha_{1}, \alpha_{2} \in T^{*}$ and $u \in C^{2 p, j}$, then the element

$$
w=\alpha_{1} \wedge \nu_{0} \pi_{p}\left(\alpha_{2} \wedge u\right)+\alpha_{2} \wedge \nu_{0} \pi_{p}\left(\alpha_{1} \wedge u\right)
$$

of $\wedge^{j+1} T^{*} \otimes R_{m}^{p}$ actually belongs to $C^{2 p+2, j+1}$. Indeed, we have

$$
\begin{aligned}
\delta \pi_{p+1}\left(\alpha_{1} \wedge \nu_{0} \pi_{p}\left(\alpha_{2} \wedge u\right)\right) & =-\alpha_{1} \wedge \delta \pi_{p+1} \nu_{0} \pi_{p}\left(\alpha_{2} \wedge u\right) \\
& =-\alpha_{1} \wedge \pi_{p}\left(\alpha_{2} \wedge u\right)=-\alpha_{1} \wedge \alpha_{2} \wedge \pi_{p} u,
\end{aligned}
$$

and so $\delta \pi_{p+1} w=0$. By (1.12), we now easily verify that the symbol

$$
\sigma(Q): S^{r+1} T^{*} \otimes C^{2 p, j} \rightarrow C^{2(p+r), j+1}
$$

of $Q$ is given by

$$
\sigma(Q)\left(\alpha_{1} \cdot \alpha_{2} \otimes u\right)=\alpha_{1} \wedge \nu_{0}\left(\alpha_{2} \wedge \pi_{p} u\right)+\alpha_{2} \wedge \nu_{0}\left(\alpha_{1} \wedge \pi_{p} u\right)
$$

when $r=1$, and by

$$
\begin{array}{r}
\sigma(Q)\left(\alpha_{1} \cdot \alpha_{2} \cdot \alpha_{3} \otimes u\right)=\frac{1}{2} \sum_{\tau \in\left(\xi_{3}\right.} \alpha_{\tau(1)} \wedge \nu_{1}\left(\alpha_{\tau(2)} \wedge \pi_{p+1} \nu_{0}\left(\alpha_{\tau(3)} \wedge \pi_{p} u\right)\right. \\
\left.+\alpha_{\tau(3)} \wedge \pi_{p+1} \nu_{0}\left(\alpha_{\tau(2)} \wedge \pi_{p} u\right)\right)
\end{array}
$$

when $r=2$, where $\alpha_{1}, \alpha_{2}, \alpha_{3} \in T^{*}, u \in C^{2 p, j}$ and $\mathfrak{G}_{3}$ is the symmetric group on 3 letters.

We denote by $\mathscr{H}^{p, j}\left(R_{m}\right)$ the sheaf of sections of the vector bundle $H^{p, j}\left(R_{m}\right)$; by means of the isomorphisms (3.4), from the operator $d_{0,2 r}$ we obtain a differential operator

$$
\tilde{d}_{0,2 r}=\mu \cdot d_{0,2 r} \cdot \mu^{-1}: \mathscr{H}^{p, j}\left(R_{m}\right) \rightarrow \mathscr{H}^{p+r, j+1}\left(R_{m}\right)
$$

of order $r+1$; we write $\tilde{d}_{0}=\tilde{d}_{0,0}$.

Assume that there are integers

$$
0=r_{0} \leqslant r_{1} \leqslant \cdots \leqslant r_{n-1} \leqslant r_{n} \leqslant m
$$

such that $H^{p, j}\left(R_{m}\right)=0$ for all $p \neq r_{j}$ and $0 \leqslant j \leqslant n, 0 \leqslant p \leqslant m$. We remark that the assumption that $H^{p, 1}\left(R_{m}\right)=0$ for $p \neq r_{1}$ implies that $g_{l}=S^{l} T^{*} \otimes E$ for $l \leqslant r_{1}$, 
and hence that $\pi_{l} R_{m}=J_{l}(T)$ for $l \leqslant r_{1}$. With these hypotheses, by the constructions of $\S 2$ and the above remarks about the operators $d_{0,2 r}$, we obtain a complex

$$
\begin{aligned}
& 0 \rightarrow \Theta \stackrel{\boldsymbol{w} \cdot j_{m}}{\rightarrow} \mathscr{C}^{0,0} / \mathscr{C}^{1,0} \stackrel{d_{0.2 r_{1}}}{\rightarrow} \mathscr{C}^{2 r_{1}, 1} / \mathscr{C}^{2 r_{1}+1,1} \\
& \stackrel{d_{0.2\left(r_{2}-r_{1}\right)}}{\rightarrow} \mathscr{C}^{2 r_{2}, 2} / \mathscr{C}^{2 r_{2}+1.2} \stackrel{d_{0.2\left(r_{3}-r_{2}\right)}}{\rightarrow} \cdots \rightarrow \mathscr{C}^{2 r_{n}, n} / \mathscr{C}^{2 r_{n}+1, n} \rightarrow 0,
\end{aligned}
$$

where $d_{0,2\left(r_{j+1}-r_{j}\right)}$ is a differential operator of order $r_{j+1}-r_{j}+1$.

We set

$$
\mathscr{L}^{j}=\mathscr{C}^{2 r_{j}, j} / \mathscr{C}^{2 r_{j}+1, j}, \quad \mathscr{L}=\bigoplus_{j} \mathscr{L}^{j},
$$

and consider the complex $\Gamma_{\Phi}\left(\mathscr{L}^{\cdot}\right)$ whose differential is induced by the operators $d_{0,2\left(r_{j+1}-r_{j}\right)}$. We remark that this complex can be obtained directly from the spectral sequence of $\Gamma_{\Phi}\left(\wedge \mathscr{T}^{*} \otimes \mathscr{R}_{m}\right)$ with the filtration $\left\{\Gamma_{\Phi}\left(\mathscr{C}^{p}\right)\right\}$. Because of the isomorphisms (3.4) and Lemma 2.1, the assumptions of Proposition 2.3 are satisfied for our two spectral sequences; this proposition on one hand tells us that the cohomology of (3.13) is isomorphic to that of (1.14) and hence that (3.13) is exact, and on the other hand gives an isomorphism

$$
\psi: H^{j}\left(\Gamma_{\Phi}\left(\mathscr{C}^{0}\right)\right) \rightarrow H^{j}\left(\Gamma_{\Phi}\left(\mathscr{L}^{\cdot}\right)\right)
$$

which can be described as follows: If $u \in \Gamma_{\Phi}\left(\mathscr{C}^{0, j}\right)$ satisfies $\nabla \hat{u}=0$, we may write $u=v+\nabla \hat{w}$, where $v \in \Gamma_{\Phi}\left(\mathscr{C}^{2 r_{j} . j}\right)$ and $w \in \Gamma_{\Phi}\left(\mathscr{C}^{0, j-1}\right)$; the mapping $\psi$ sends the cohomology class of $u$ in $H^{j}\left(\Gamma_{\Phi}\left(\mathscr{C}^{0}\right)\right)$ into the cohomology class of the element $\varpi v$ of $\Gamma_{\Phi}\left(\mathscr{L}^{j}\right)$ in $H^{j}\left(\Gamma_{\Phi}\left(\mathscr{L}^{\cdot}\right)\right)$.

We set

$$
\begin{gathered}
\mathscr{Z}^{p, j}=\left\{u \in \mathscr{C}^{p, j} \mid \nabla^{\hat{u}} u=0\right\}, \\
\mathscr{Z}^{j}=\left\{u \in \mathscr{C}^{r_{j}, j} / \mathscr{C}^{r_{j}+1, j} \mid d_{0,2\left(r_{j+1}-r_{j}\right)} u=0\right\} ;
\end{gathered}
$$

for $p \geqslant q$, let $i_{q}: \mathscr{Z}^{2 p, j} \rightarrow \mathscr{Z}^{2 q, j}$ denote the natural inclusion. If $\boldsymbol{w}$ is the projection $C^{2 r_{j}, j} \rightarrow C^{2 r_{j}, j} / C^{2 r_{j}+1, j}$, it is easily verified that $\varpi\left(\mathscr{Z}^{2 r_{j}, j}\right) \subset \mathscr{Z}^{j}$. Thus for $j \geqslant 0$, we have a commutative diagram

$$
\begin{array}{ccccccc}
0 \rightarrow & \mathscr{Z}^{2 r_{j}, j} & \rightarrow & \mathscr{C}^{2 r_{j}, j} & \stackrel{\nabla^{0}}{\rightarrow} & \mathscr{Z}^{2 r_{j}, j+1} & \rightarrow 0 \\
& \downarrow \varpi & & \downarrow \downarrow & & & \\
& & & & & \\
0 \rightarrow & \mathscr{Z}^{j} & \rightarrow & \mathscr{C}^{2 r_{j}, j} / \mathscr{C}^{2 r_{j}+1, j} & \stackrel{d_{0,2\left(r_{j+1}-r_{j}\right)}}{\rightarrow} & \mathscr{Z}^{j+1} & \rightarrow 0 ;
\end{array}
$$

according to Lemma 3.1 and the exactness of (3.13), it is also exact. This diagram now determines a morphism $\varphi: \mathscr{Z}^{2 r_{j}, j+1} \rightarrow \mathscr{Z}^{j+1}$ such that $d_{0,2\left(r_{j+1}-r_{j}\right)} \varpi=\varphi \nabla^{\wedge}$. Then the diagram

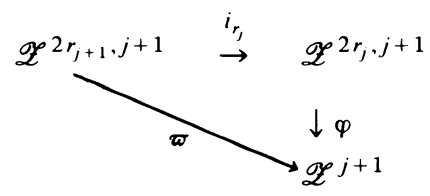


commutes. Indeed, let $u \in \mathscr{Z}^{2 r_{1+1}, j+1}$; we may write $u=\nabla \hat{v}$ for some $v \in \mathscr{C}^{2 r_{1}, j}$. Then, since $\nabla \hat{v} \in \mathscr{C}^{2 r_{1+1}, j+1}$, we have

$$
\varphi u=d_{0,2\left(r_{1+1}-r_{1}\right)} \varpi v=\varpi \nabla \hat{v}=\varpi u .
$$

From the diagram (3.14), we obtain the commutative diagram

$$
\begin{array}{ccc}
H_{\Phi}^{i}\left(X, \mathscr{Z}^{2 r_{1}, j+1}\right) & \stackrel{\delta}{\rightarrow} & H_{\Phi}^{i+1}\left(X, \mathscr{Z}^{2 r_{,}, j}\right) \\
\downarrow \varphi & & \downarrow \varpi \\
H_{\Phi}^{i}\left(X, \mathscr{Z}^{j+1}\right) & \stackrel{\delta}{\rightarrow} & H_{\Phi}^{i+1}\left(X, \mathscr{Z}^{j}\right)
\end{array}
$$

where $\delta$ are connecting homomorphisms; therefore by the commutativity of diagram (3.15) and the exactness of the sequence

$$
0 \rightarrow \mathscr{Z}^{0, j} \rightarrow \mathscr{C}^{0, j} \stackrel{\nabla^{-}}{\rightarrow} \mathscr{Z}^{0, j+1} \rightarrow 0,
$$

we have the commutative diagram:

$$
\begin{array}{ccc}
H_{\Phi}^{i}\left(X, \mathscr{Z}^{0, j+1}\right) & \stackrel{\delta}{\rightarrow} & H_{\Phi}^{i+1}\left(X, \mathscr{Z}^{0, j}\right) \\
\uparrow i_{0} & & \uparrow i_{0} \\
H_{\Phi}^{i}\left(X, \mathscr{Z}^{2 r_{1+1}, j+1}\right) & \stackrel{\delta \cdot i_{r_{1}}}{\rightarrow} & H_{\Phi}^{i+1}\left(X, \mathscr{Z}^{2 r_{,}, j}\right) \\
\downarrow \downarrow & & \downarrow \varpi \\
H_{\Phi}^{i}\left(X, \mathscr{Z}^{j+1}\right) & \stackrel{\delta}{\rightarrow} & H_{\Phi}^{i+1}\left(X, \mathscr{Z}^{j}\right) .
\end{array}
$$

Thus by composing the horizontal arrows of this last diagram, we obtain a commutative diagram

$$
\begin{aligned}
& \begin{array}{ccccc}
\Gamma_{\Phi}\left(\mathscr{Z}^{0, j}\right) & \rightarrow & H_{\Phi}^{j}\left(X, \mathscr{Z}^{0,0}\right) & \stackrel{j_{m}}{\rightleftarrows} & H_{\Phi}^{j}(X, \Theta) \\
\uparrow i_{0} & & \uparrow \text { id } & & \uparrow \text { id }
\end{array} \\
& \begin{array}{ccccc}
\Gamma_{\Phi}\left(\mathscr{Z}^{2 r_{j}, j}\right) & \rightarrow & H_{\Phi}^{j}\left(X, \mathscr{Z}^{0,0}\right) & \stackrel{j_{m}}{\rightleftarrows} & H_{\Phi}^{j}(X, \Theta) \\
\downarrow \varpi & & \downarrow \varpi & & \downarrow \text { id }
\end{array} \\
& \Gamma_{\Phi}\left(\mathscr{Z}^{j}\right) \rightarrow H_{\Phi}^{j}\left(X, \mathscr{Z}^{0}\right) \stackrel{\varpi \tilde{j}_{m}}{\leftarrow} H_{\Phi}^{j}(X, \Theta)
\end{aligned}
$$

for $j \geqslant 0$. From the commutativity of diagram (3.16) and the definition of the mapping $\psi$, we infer that the diagram

$$
\begin{array}{cccc}
H_{\Phi}^{j}(X, \Theta) & \rightarrow & H^{j}\left(\Gamma_{\Phi}\left(\mathscr{C}^{0}\right)\right) \\
\downarrow \text { id } & & \downarrow \psi \\
H_{\Phi}^{j}(X, \Theta) & \rightarrow & H^{j}\left(\Gamma_{\Phi}\left(\mathscr{L}^{\cdot}\right)\right),
\end{array}
$$

whose horizontal arrows are the isomorphisms determined by the resolutions (1.14) and (3.13) of $\Theta$, commutes. 
Let $x \in X$ and $\alpha \in T_{x}^{*}$, with $\alpha \neq 0$. If $P$ is a differential operator, we consider its symbol $\sigma_{\alpha}(P)$ at $\alpha$ as defined in [7, §12] (see also [8]). The sequence (1.14) (with $\Theta$ omitted) is an elliptic complex. We set $L_{x}^{j}=\left(C^{2 r_{j}, j} / C^{2 r_{j}+1, j}\right)_{x}$ and consider the complex $L_{x}^{\cdot}=\oplus_{j} L_{x}^{j}$ whose differential is induced by the mappings $\sigma_{\alpha}\left(d_{0,2\left(r_{j+1}-r_{j}\right)}\right)$. It is easily seen that this complex can be obtained directly from the spectral sequence of $\left(\wedge T^{*} \otimes R_{m}\right)_{x}$ with the differential $\sigma_{\alpha}\left(\nabla^{\wedge}\right)$ and the filtration $\left\{C_{x}^{p}\right\}$. Now by Proposition 2.3, since (1.14) is an elliptic complex, the cohomology of $L_{x}$ vanishes and so (3.13) (with $\Theta$ omitted) is also an elliptic complex.

We write $E^{j}=H^{r_{j}, j}\left(R_{m}\right)$ for $0 \leqslant j \leqslant n$; by means of the isomorphisms (3.4), we obtain from the complex (3.13) the exact sequence

$$
0 \rightarrow \Theta \stackrel{i}{\rightarrow} \mathscr{E}^{0} \stackrel{D_{0}}{\rightarrow} \mathscr{E}^{1} \stackrel{D_{1}}{\rightarrow} \mathscr{E}^{2} \rightarrow \cdots \rightarrow \mathscr{E}^{n} \rightarrow 0,
$$

where $D_{j}=\tilde{d}_{0,2\left(r_{j+1}-r_{j}\right)}$ is a differential operator of order $r_{j+1}-r_{j}+1$; here $E^{0}=E$ and $i$ is the inclusion of $\Theta$ in $\mathscr{E}$, since $\mu \varpi: R_{m} \rightarrow E^{0}$ is equal to $\pi_{0}$. We let $\mathscr{E} \cdot$ denote the complex $\oplus_{j} \mathscr{E}^{j}$ with the differential induced by the differential operators $D_{j}$. We have an isomorphism

$$
\rho: H^{j}\left(\Gamma_{\Phi}\left(\mathscr{C}^{0}\right)\right) \rightarrow H^{j}\left(\Gamma_{\Phi}\left(\mathscr{E}^{\bullet}\right)\right)
$$

which can be described as follows. If $u \in \Gamma_{\Phi}\left(\mathscr{C}^{0, j}\right)$ satisfies $\nabla^{\wedge} u=0$, then we may write $u=v+\nabla^{\wedge} w$, where $v \in \Gamma_{\Phi}\left(\mathscr{C}^{2 r_{j}, j}\right)$ and $w \in \Gamma_{\Phi}\left(\mathscr{C}^{0, j-1}\right)$; the mapping $\rho$ sends the class of $u$ in $H^{j}\left(\Gamma_{\Phi}\left(\mathscr{C}^{0}\right)\right)$ into the class of $\rho_{r_{j}} v$ in $H^{j}\left(\Gamma_{\Phi}(\mathscr{E} \bullet)\right)$.

From the commutativity of diagram (3.17), we deduce

THEOREM 3.1. Let $R_{m} \subset J_{m}(E)$ be a formally integrable differential equation, with $g_{m+1}=0$. Assume that $\pi_{0}: R_{m} \rightarrow E$ is surjective, that $R_{m}^{l}$ is a vector bundle for $0 \leqslant l \leqslant m$, and that the mappings (3.2) are of constant rank for all $j, l \geqslant 0$. Assume moreover that there are integers $0=r_{0} \leqslant r_{1} \leqslant \cdots \leqslant r_{n-1} \leqslant r_{n} \leqslant m$ such that $H^{p, j}\left(R_{m}\right)=0$ for all $p \neq r_{j}$ and $0 \leqslant j \leqslant n, 0 \leqslant p \leqslant m$. Then we have the resolution (3.18) of $\Theta$ which is an elliptic complex, and the diagram

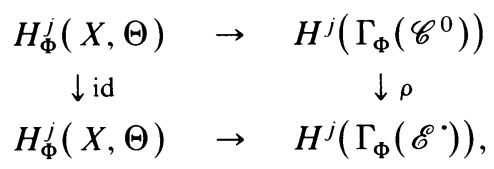

whose horizontal arrows are the isomorphisms determined by the resolutions (1.14) and (3.18) of $\Theta$, is commutative.

4. Semisimple graded Lie algebras. Let $\mathfrak{g}=\oplus_{p \in \mathbf{Z}} \mathfrak{g}_{p}$ be a real finite-dimensional Lie algebra having the following properties:

(i) $\mathfrak{g}_{p}=0$ for $p \leqslant-2$;

(ii) $\left[\mathfrak{g}_{p}, \mathfrak{g}_{q}\right] \subset \mathfrak{g}_{p+q}$ for $p, q \in \mathbf{Z}$.

We consider the Lie algebra cohomology $H(\mathfrak{g})=H\left(\mathfrak{g}_{-1}, \mathfrak{g}\right)$ of the abelian Lie algebra $\mathfrak{g}_{-1}$ with values in the $\mathfrak{g}_{-1}$-module $g$ corresponding to the adjoint representation of $\mathfrak{g}_{-1}$ on $\mathfrak{g}$. Let

$$
C^{p, j}(\mathfrak{g})=\wedge^{j} \mathfrak{g}_{-1}^{*} \otimes \mathfrak{g}_{p-1}
$$


and define

$$
\delta: C^{p, j}(\mathfrak{g}) \rightarrow C^{p-1, j+1}(\mathfrak{g})
$$

by

$$
(\delta u)\left(\xi_{1}, \ldots, \xi_{j+1}\right)=\sum_{i=1}^{q+1}(-1)^{i+1}\left[\xi_{i}, u\left(\xi_{1}, \ldots, \hat{\xi}_{i}, \ldots, \xi_{j+1}\right)\right]
$$

for $u \in C^{p, j}(\mathfrak{g})$ and $\xi_{1}, \ldots, \xi_{j+1} \in \mathfrak{g}_{-1}$. Then

$$
C^{p+1, j-1}(\mathfrak{g}) \stackrel{\delta}{\rightarrow} C^{p, j}(\mathfrak{g}) \stackrel{\delta}{\rightarrow} C^{p-1, j+1}(\mathfrak{g})
$$

is a complex whose cohomology we denote by $H^{p, j}(\mathrm{~g})$. Then

$$
H(\mathfrak{g})=\bigoplus_{p, j \in \mathbf{Z}} H^{p, j}(\mathfrak{g}), \quad H^{j}(\mathfrak{g})=\bigoplus_{p \in \mathbf{Z}} H^{p, j}(\mathfrak{g}) .
$$

If $\left\{\xi_{1}, \ldots, \xi_{n}\right\}$ is a basis of $\mathfrak{g}_{-1}$ and $\left\{\alpha_{1}, \ldots, \alpha_{n}\right\}$ is the dual basis of $\mathfrak{g}_{-1}^{*}$, then it is easily seen that

$$
\delta(\alpha \otimes \xi)=\sum_{i=1}^{n}\left(\alpha_{i} \wedge \alpha\right) \otimes\left[\xi_{i}, \xi\right]
$$

for $\alpha \in \wedge^{j} \mathfrak{g}_{-1}^{*}$ and $\xi \in \mathfrak{g}_{p-1}$. Since

$$
\delta(\alpha \wedge u)=(-1)^{j} \alpha \wedge \delta u
$$

for $\alpha \in \Lambda^{j} \mathfrak{g}_{-1}^{*}$ and $u \in \Lambda^{\prime} \mathfrak{g}_{-1}^{*} \otimes \mathfrak{g}_{p-1}$, the multiplication

$$
\mathfrak{g}_{-1}^{*} \otimes \wedge^{j} \mathfrak{g}_{-1}^{*} \rightarrow \wedge^{j+1} \mathfrak{g}_{-1}^{*}
$$

sending $\alpha \otimes \omega$ into $\alpha \wedge \omega$, induces a mapping

$$
\mathfrak{g}_{-1}^{*} \otimes H^{p, j}(\mathfrak{g}) \rightarrow H^{p, j+1}(\mathfrak{g}) \text {. }
$$

Clearly the mapping $\mathrm{g}_{-1}^{*} \otimes H^{0, j}(\mathrm{~g}) \rightarrow H^{0, j+1}(\mathrm{~g})$ is surjective for $j \geqslant 0$.

Let $B$ denote the Killing form of $g$ and consider the mapping

$$
\left(\wedge^{i} \mathfrak{g}_{-1}^{*} \otimes \mathfrak{g}\right) \otimes\left(\wedge^{j} \mathfrak{g}_{-1}^{*} \otimes \mathfrak{g}\right) \rightarrow \wedge^{i+j} \mathfrak{g}_{-1}^{*}
$$

sending $u \otimes v$ into $\langle u, v\rangle$, determined by

$$
\langle u, v\rangle=B(\xi, \eta) \alpha \wedge \beta
$$

if $u=\alpha \otimes \xi$ and $v=\beta \otimes \eta$, where $\alpha \in \wedge^{i} \mathfrak{g}_{-1}^{*}, \beta \in \wedge^{j} \mathfrak{g}_{-1}^{*}$ and $\xi, \eta \in \mathfrak{g}$.

Lemma 4.1. For $u \in \wedge^{i} \mathfrak{g}{ }_{-1}^{*} \otimes \mathfrak{g}$ and $v \in \wedge^{j} \mathfrak{g}_{-1}^{*} \otimes \mathfrak{g}$, we have

$$
\langle\delta u, v\rangle=(-1)^{i+1}\langle u, \delta v\rangle \text {. }
$$

Proof. Suppose that $u=\alpha \otimes \xi$ and $v=\beta \otimes \eta$, with $\alpha \in \wedge^{i} \mathfrak{g}_{-1}^{*}, \beta \in \wedge^{j} \mathfrak{g}_{-1}^{*}$ and $\xi, \eta \in \mathfrak{g}$. If $\left\{\xi_{1}, \ldots, \xi_{n}\right\}$ is a basis of $\mathfrak{g}_{-1}$ and $\left\{\alpha_{1}, \ldots, \alpha_{n}\right\}$ is the dual basis of $\mathfrak{g}_{-1}^{*}$, by (4.2) we have

$$
\begin{aligned}
\langle\delta(\alpha \otimes \xi), \beta \otimes \eta\rangle & =\sum_{k=1}^{n} B\left(\left[\xi_{k}, \xi\right], \eta\right) \alpha_{k} \wedge \alpha \wedge \beta \\
& =(-1)^{i+1} \sum_{k=1}^{n} B\left(\xi,\left[\xi_{k}, \eta\right]\right) \alpha \wedge \alpha_{k} \wedge \beta \\
& =(-1)^{i+1}\left\langle\alpha \otimes \xi,\left(\alpha_{k} \wedge \beta\right) \otimes\left[\xi_{k}, \eta\right]\right\rangle \\
& =(-1)^{i+1}\langle\alpha \otimes \xi, \delta(\beta \otimes \eta)\rangle .
\end{aligned}
$$


According to Lemma 4.1, the mapping, sending $u \otimes v$, with $u \in C^{p, i}(\mathfrak{g})$ and $v \in C^{q . j}(\mathfrak{g})$, into $\langle u, v\rangle \in \wedge^{i+j} \mathfrak{g}_{-1}^{*}$, induces by passage to the quotient a well-defined pairing

$$
H^{p, i}(\mathfrak{g}) \otimes H^{q, j}(\mathfrak{g}) \rightarrow \wedge^{i+j} \mathfrak{g}_{-1}^{*}
$$

sending $u \otimes v$ into $\langle u, v\rangle$; if $u \in H^{p, i}(\mathfrak{g})$ and $v \in H^{q, j}(\mathfrak{g})$, we have $\langle u, v\rangle=$ $(-1)^{i j}\langle v, u\rangle$.

The next proposition is proved in [12].

Proposition 4.1. If $\mathrm{g}$ is semisimple, we have:

(i) $\mathfrak{g}_{p}=0$ for $p \geqslant 2$;

(ii) $B_{\mid \mathrm{g}_{0}}$ is nondegenerate;

(iii) $\mathfrak{g}_{1}$ is the vector space dual to $\mathfrak{g}_{-1}$ under the pairing

$$
(\xi, \eta) \mapsto B(\xi, \eta) \quad \text { for } \xi \in \mathfrak{g}_{-1}, \eta \in \mathfrak{g}_{1} ;
$$

(iv) there exists a unique element $e \in \mathrm{g}$ such that

$$
[e, \xi]=i \xi \quad \text { for } \xi \in \mathrm{g}_{i}, i=-1,0,1 ;
$$

this element e belongs to $\mathfrak{g}_{0}$; if $\sigma: \mathfrak{g} \rightarrow \mathfrak{g}$ is a Cartan involution, then $\sigma(e)=-e$.

If $\mathfrak{g}$ is semisimple, let $\tau: \mathfrak{g} \rightarrow \mathfrak{g}$ be the automorphism of $\mathrm{g}$ defined by

$$
\tau(\xi+\eta+\zeta)=-\xi+\eta-\zeta
$$

for $\xi \in \mathrm{g}_{-1}, \eta \in \mathrm{g}_{0}$ and $\zeta \in \mathrm{g}_{1}$.

Because of Proposition 3.2 of [16], the proof of the following proposition is essentially the same as that of Lemma 6 of [12] and shall be omitted.

Proposition 4.2. Assume that $\mathrm{g}$ is semisimple and let e be the element of $\mathrm{g}_{0}$ given by Proposition 4.1(iv). If $\sigma$ is an involutive automorphism of $\mathrm{g}$ such that $\tau \sigma=\sigma \tau$ and $\sigma(e)=-e$, then

$$
\sigma\left(\mathfrak{g}_{p}\right)=\mathfrak{g}_{-p} \text { for } p=-1,0,1 .
$$

We also recall the following result of [12].

Proposition 4.3. Assume that for $\xi \in \mathfrak{g}_{p}$, with $p \geqslant 0$, the condition $\left[\mathfrak{g}_{-1}, \xi\right]=0$ implies that $\xi=0$. Then $\mathrm{g}$ is a simple Lie algebra if and only if the representation ad: $\mathfrak{g}_{0} \rightarrow \mathfrak{g}_{-1}^{*} \otimes \mathfrak{g}_{-1}$ is irreducible and $\mathfrak{g}_{1} \neq 0$.

Assume that $\mathfrak{g}$ is semisimple; we know that $\mathfrak{g}_{p}=0$ for $p>2$. According to Lemma 10.2 of [1] (see Lemma 1.3), there exists a Cartan involution $\sigma$ of $\mathfrak{g}$ such that $\sigma \tau=\tau \sigma$. By Propositions 4.1 and 4.2, we see that (4.6) holds. Then the mapping $B_{\sigma}$ : $\mathfrak{g} \otimes \mathfrak{g} \rightarrow \mathbf{R}$, defined by

$$
B_{\sigma}(\xi, \eta)=-B(\xi, \sigma(\eta))
$$

for $\xi, \eta \in \mathfrak{g}$, determines a symmetric positive definite form on $\mathfrak{g}$ and scalar products on $\mathfrak{g}_{-1}$ and $\Lambda \mathfrak{g}_{-1}^{*}$; we denote by $(, \quad)$ the scalar product induced by $B_{\sigma}$ on $\wedge \mathrm{g}_{-1}^{*} \otimes \mathrm{g}_{-1}$. Let

$$
\delta^{*}: \wedge^{j+1} \mathfrak{g}_{-1}^{*} \otimes \mathfrak{g} \rightarrow \wedge^{j} \mathfrak{g}_{-1}^{*} \otimes \mathfrak{g}
$$

be the adjoint of $\delta$ and set

$$
\mathbf{H}^{p, j}=\left\{u \in \wedge^{j} \mathfrak{g}_{-1}^{*} \otimes \mathfrak{g}_{p-1} \mid \delta u=0, \delta^{*} u=0\right\} .
$$


We have a natural mapping

$$
\mathbf{H}^{p, j} \rightarrow H^{p, j}
$$

sending $u$ into its cohomology class.

If $\mathfrak{g}_{-1}$ is oriented and $\Omega \in \wedge^{n} \mathfrak{g}_{-1}^{*}$ is the corresponding volume element, where $n=\operatorname{dim} \mathfrak{g}_{-1}$, let

$$
*: \wedge^{j} \mathfrak{g}_{-1}^{*} \otimes \mathfrak{g} \rightarrow \wedge^{n-j} \mathfrak{g}_{-1}^{*} \otimes \mathfrak{g}
$$

be the mapping defined in terms of the Hodge $*$-isomorphism by

$$
*(\alpha \otimes \xi)=-(* \alpha) \otimes \sigma(\xi)
$$

for $\alpha \in \wedge^{j} \mathfrak{g}_{-1}^{*}$ and $\xi \in \mathfrak{g}$. Then (1.24) and (1.25) hold for $u, v \in \wedge^{j} \mathfrak{g}_{-1}^{*} \otimes \mathfrak{g}$. Therefore, by Lemma 4.1, for $u \in \wedge^{j} \mathfrak{g}_{-1}^{*} \otimes \mathfrak{g}$ and $v \in \wedge^{j+1} \mathfrak{g}_{-1}^{*} \otimes \mathfrak{g}$, we have

$$
\begin{aligned}
(\delta u, v) \Omega & =\langle\delta u, * v\rangle=(-1)^{j+1}\langle u, \delta * v\rangle \\
& =(-1)^{n j+1}\langle u, * * \delta * v\rangle=(-1)^{n j+1}(u, * \delta * v) \Omega,
\end{aligned}
$$

and so the adjoint (4.7) of $\delta$ is equal to $(-1)^{n j+1} * \delta *$. According to the standard Hodge theory and (4.6), the mapping (4.8) is an isomorphism and the mapping (4.9) induces an isomorphism $*: \mathbf{H}^{p, j} \rightarrow \mathbf{H}^{2-p, n-j}$ for $p=0,1,2$ and $0 \leqslant j \leqslant n$. We deduce

Proposition 4.4. If $\mathfrak{g}$ is semisimple, the pairing

$$
H^{p, j}(\mathrm{~g}) \otimes H^{2-p, n-j}(\mathrm{~g}) \rightarrow \wedge^{n} \mathrm{~g}_{-1}^{*}
$$

given by (4.5) is nondegenerate.

The assertion of Proposition 4.4 in the case of $\mathfrak{g}=\mathfrak{s} \mathfrak{o}(n+1,1)$, for $n \geqslant 3$, is already given by [7, Proposition 10.1] (see \$7).

REMARK 4.1. If $\mathfrak{g}$ is semisimple, this cohomology $H(\mathfrak{g})$ of $\mathfrak{g}$ can be computed using results of Kostant [13] (see also [16]).

By (4.3), we see that $\oplus_{j \geqslant 0} H^{0, j}(\mathfrak{g})$ is a graded $\wedge \mathfrak{g}_{-1}^{*}$-module generated in degree 0 . Hence there exists an integer $0 \leqslant k \leqslant n$ such that $H^{0, j}(\mathfrak{g}) \neq 0$ for $0 \leqslant j \leqslant k$, and $H^{0, j}(\mathfrak{g})=0$ for $j>k$; if $\mathfrak{g}$ is semisimple, by Proposition 4.4 we have $H^{2, j}(\mathfrak{g})=0$ for $j<n-k$, and $H^{2, j}(\mathrm{~g}) \neq 0$ for $n-k \leqslant j \leqslant n$.

5. Selfadjoint resolutions. In this section, we suppose that $R_{m} \subset J_{m}(T)$ is a formally integrable and formally transitive Lie equation of order $m$, with $g_{m+1}=0$. We assume that $R_{m}^{l}$ is a vector bundle and that the mappings (3.2) are of constant rank for all $j, l \geqslant 0$; according to Proposition 5.4 of [9], the fact that $R_{m}$ is a formally integrable and formally transitive Lie equation implies that these conditions hold on each connected component of $X$.

If we set $R_{m}^{\prime}=R_{m}$ for all $l \leqslant-1$, under the bracket (1.16), we have

$$
\left[R_{m}^{p}, R_{m}^{q}\right] \subset R_{m}^{p+q}
$$

for all $p, q \in \mathbf{Z}$. For $x \in X$, we see that $\left\{R_{m, x}^{l}\right\}_{l \in \mathbf{Z}}$ is a filtered Lie algebra structure on $R_{m, x}$, and the associated graded Lie algebra

$$
\operatorname{gr} R_{m, x}=\bigoplus_{p \in \mathbf{Z}} R_{m, x}^{p} / R_{m, x}^{p+1}
$$


can be identified with the fiber $g_{x}$ at $x$ of the vector bundle $g=\oplus_{p \in \mathbf{Z}} g_{p+1}$, where $g_{p}=0$ for $p \leqslant-1$ or $p \geqslant m+1$. The graded Lie algebra $\mathfrak{g}=g_{x}$, with $\mathfrak{g}_{p}=g_{p+1, x}$, satisfies conditions (i) and (ii) of $\S 4$.

If $\xi \in T$ and $u \in g_{k}$, we have

$$
[\xi, u]=\delta_{\xi} u
$$

if $u, v \in g_{1}$, we have

$$
[u, v]=v \circ u-u \circ v,
$$

considering $u, v$ as elements of $T^{*} \otimes T=\operatorname{Hom}(T, T)$; finally, if $m \geqslant 2, u \in g_{2}$ and $v \in g_{1}$, then $[u, v] \in g_{2}$ is given by

$$
[u, v](\xi, \eta)=v(u(\xi, \eta))-u(v(\xi), \eta)-u(\xi, v(\eta))
$$

for $\xi, \eta \in T$, considering $u,[u, v]$ as elements of $S^{2} T^{*} \otimes T$ and $v$ as an element of $T^{*} \otimes T=\operatorname{Hom}(T, T)$. From (5.2), it follows that the operator $\delta$ of the complex (3.1) at $x$ is equal to the coboundary operator $\delta$ given by (4.1), and so $H^{l, j}\left(R_{m}\right)_{x}=$ $H^{l, j}(\mathrm{~g})$. According to Proposition 5.4 of [9], $g$ is a locally trivial bundle of Lie algebras.

Consider the Killing form $B$ of $R_{m}$ of $\S 1$ and the mapping $\bar{B}: g \otimes g \rightarrow \mathbf{R}$, whose restriction to the fiber at $x \in X$ is the Killing form $\bar{B}_{x}$ of the Lie algebra $g_{x}$. Then from (5.1), if $m=2$ we deduce that

$$
B\left(g_{2}, R_{2}^{0}\right)=0
$$

and hence that

$$
B(u, v)=\bar{B}\left(\pi_{1} u, \pi_{1} v\right) \quad \text { for } u, v \in R_{2}^{0}
$$

and

$$
B(u, v)=\bar{B}\left(\pi_{0} u, v\right) \quad \text { for } u \in R_{2}, \quad v \in g_{2} .
$$

Lemma 5.1. Let $x \in X$. Suppose that $\mathrm{gr} R_{m, x}$ is a semisimple Lie algebra. Then $g_{l, x}=0$ for $l \geqslant 3$ and $g_{2, x}$ is isomorphic to $T_{x}^{*}$; moreover $R_{m, x}$ is a semisimple Lie algebra.

Proof. The first two assertions are given by Proposition 4.1. Thus we may assume that $m=2$. Let $u \in R_{2, x}$ with $B(u, v)=0$ for all $v \in R_{2, x}$. For $v \in g_{2, x}$, by (5.7) we have $\bar{B}\left(\pi_{0} u, v\right)=0$ and so $\pi_{0} u=0$ by Proposition 4.1(iii). Now $u \in R_{2, x}^{0}$, and for $v \in R_{2, x}^{0}$ by (5.6) we see that $\bar{B}\left(\pi_{1} u, \pi_{1} v\right)=0$; thus by Proposition 4.1(ii), we have $\pi_{1} u=0$. Finally, for $v \in R_{2, x}$, by (5.7) we obtain $\bar{B}\left(u, \pi_{0} v\right)=0$ and hence $u=0$.

For the remainder of this section, we suppose that $m=2$. Consider the pairings (1.17) and (4.4), with $\mathfrak{g}=g_{x}$ and $g_{-1}=T_{x}$, where $x \in X$. For $u \in C^{2 p, i}$ and $v \in C^{5-2 p, j}$, with $p=1,2$, we have

$$
\langle u, v\rangle=0
$$

indeed, we have $\delta \pi_{p} u=0$ and $\pi_{2-p} v=\delta w$ for some $w \in \Lambda^{j-1} T^{*} \otimes g_{3-p}$, and hence by (5.6), (5.7) and Lemma 4.1 we see that

$$
\langle u, v\rangle=\left\langle\pi_{p} u, \pi_{2-p} v\right\rangle=\left\langle\pi_{p} u, \delta w\right\rangle=(-1)^{i+1}\left\langle\delta \pi_{p} u, w\right\rangle=0 .
$$


Therefore for $p \geqslant 0$, the diagram

$$
\begin{array}{cccc}
C^{2 p, i} \otimes C^{4-2 p, j} & \rightarrow & \wedge^{i+j} T^{*} \\
\downarrow \rho_{p} \otimes \rho_{2, p} & & \downarrow \text { id } \\
H^{p, i}\left(R_{m}\right) \otimes H^{2-p, j}\left(R_{m}\right) & \rightarrow & \wedge^{i+j} T^{*},
\end{array}
$$

whose horizontal arrows are given by the pairings (1.17) and (4.5), with $g=g_{x}$ for $x \in X$, is commutative.

The following lemma follows directly from the commutativity of (5.9) and Lemma 1.2 .

LeMmA 5.2. For $u \in \mathscr{H}^{p, i}\left(R_{2}\right)$ and $v \in \mathscr{H}^{2-p, j}\left(R_{2}\right)$, we have

$$
\left\langle\tilde{d}_{0} u, v\right\rangle+(-1)^{i}\left\langle u, \tilde{d}_{0} v\right\rangle=d\langle u, v\rangle .
$$

LemMA 5.3. Let $r=1$ and $p=0,1$, or $r=2$ and $p=0$. Assume that

$$
\begin{array}{ll}
H^{q, i+1}\left(R_{m}\right)=0 & \text { for } p \leqslant q<p+r, \\
H^{q . i}\left(R_{m}\right)=0 & \text { for } p<q \leqslant p+r, \\
H^{q, j+1}\left(R_{m}\right)=0 & \text { for } 2-r-p \leqslant q<2-p, \\
H^{q . j}\left(R_{m}\right)=0 & \text { for } 2-r-p<q \leqslant 2-p .
\end{array}
$$

Then there exists a differential operator

$$
\alpha: \mathscr{H}^{p, i}\left(R_{2}\right) \oplus \mathscr{H}^{2-r-p . j}\left(R_{2}\right) \rightarrow \wedge^{i+j} T^{*}
$$

of order $r$ such that

$$
\left\langle\tilde{d}_{0,2 r} u, v\right\rangle+(-1)^{i}\left\langle u, \tilde{d}_{0,2 r} v\right\rangle=d \alpha(u \oplus v)
$$

for all $u \in \mathscr{H}^{p, i}\left(R_{2}\right)$ and $v \in \mathscr{H}^{2-r-p, j}\left(R_{2}\right)$.

Proof. We write $s=2-r-p$. As we have seen in $\S 3$, there exist differential operators

$$
Q^{\prime}: \mathscr{C}^{2 p, i} \rightarrow \mathscr{C}^{2 p, i}, \quad Q^{\prime \prime}: \mathscr{C}^{2 s, j} \rightarrow \mathscr{C}^{2 s, j}
$$

of order $r$ such that

$$
\nabla^{\wedge} Q^{\prime} u^{\prime} \in C^{2(p+r), i+1}, \quad \nabla^{\wedge} Q^{\prime \prime} u^{\prime \prime} \in C^{2(2-p), j+1}
$$

and

$$
u^{\prime}-Q^{\prime} u^{\prime} \in \mathscr{C}^{2 p+1, i}, \quad u^{\prime \prime}-Q^{\prime \prime} u^{\prime \prime} \in \mathscr{C}^{2 s+1, j}
$$

for all $u^{\prime} \in \mathscr{C}^{2 p, i}$ and $u^{\prime \prime} \in \mathscr{C}^{2 s, j}$. Let

$$
\chi^{\prime}: H^{p, i}\left(R_{2}\right) \rightarrow C^{2 p, i}, \quad \chi^{\prime \prime}: H^{s, j}\left(R_{2}\right) \rightarrow C^{2 s, j}
$$

be morphisms of vector bundles such that $\rho_{p} \chi^{\prime}=$ id and $\rho_{s} \chi^{\prime \prime}=$ id. Let $u \in$ $\mathscr{H}^{p, i}\left(R_{2}\right)$ and $v \in \mathscr{H}^{s, j}\left(R_{2}\right)$; we write $\tilde{u}=\chi^{\prime} u$ and $\tilde{v}=\chi^{\prime \prime} v$. Then $\tilde{d}_{0,2 r} u=$ $\rho_{p+r} \nabla^{\wedge} Q^{\prime} \tilde{u}$ and $\tilde{d}_{0,2 r} v=\rho_{2-p} \nabla^{\wedge} Q^{\prime \prime} \tilde{v}$. According to (5.8), we have

$$
\left\langle\nabla^{\wedge} Q^{\prime} \tilde{u}, \tilde{v}-Q^{\prime} \tilde{v}\right\rangle=0, \quad\left\langle\tilde{u}-Q^{\prime} \tilde{u}, \nabla^{\wedge} Q^{\prime \prime} \tilde{u}\right\rangle=0 .
$$


Hence by the commutativity of diagram (5.9) and Lemma 1.2, we obtain

$$
\begin{aligned}
\left\langle\tilde{d}_{0,2 r} u, v\right\rangle+(-1)^{i}\left\langle u, \tilde{d}_{0,2 r} v\right\rangle & =\left\langle\nabla^{\wedge} Q^{\prime} \tilde{u}, \tilde{v}\right\rangle+(-1)^{i}\left\langle\tilde{u}, \nabla^{\wedge} Q^{\prime \prime} \tilde{v}\right\rangle \\
& =\left\langle\nabla^{\wedge} Q^{\prime} \tilde{u}, Q^{\prime \prime} \tilde{v}\right\rangle+(-1)^{i}\left\langle Q^{\prime} \tilde{u}, \nabla^{\prime} Q^{\prime \prime} \tilde{v}\right\rangle \\
& =d\left\langle Q^{\prime} \tilde{u}, Q^{\prime \prime} \tilde{v}\right\rangle=d\left\langle Q^{\prime} \chi^{\prime} u, Q^{\prime \prime} \chi^{\prime \prime} v\right\rangle .
\end{aligned}
$$

We now see that the desired result holds if the differential operator $\alpha$ is defined by

$$
\alpha(u \oplus v)=\left\langle Q^{\prime} \chi^{\prime} u, Q^{\prime \prime} \chi^{\prime \prime} v\right\rangle .
$$

If $\mathscr{A}$ is a sheaf over $X$ and if $\Phi$ is the family of all closed subsets of $X$, we write $\Gamma(\mathscr{A})=\Gamma_{\Phi}(\mathscr{A})$; if $\Phi$ is the family of all compact subsets of $X$, we write $\Gamma_{0}(\mathscr{A})=$ $\Gamma_{\Phi}(\mathscr{A})$.

Assume that there is an integer $0 \leqslant k \leqslant(n+1) / 2$ such that

$$
\begin{gathered}
H^{0, j}\left(R_{2}\right)=0 \text { for } j>k, \\
H^{1, j}\left(R_{2}\right)=0 \text { for } j \leqslant k \text { and } j \geqslant n-k, \\
H^{2, j}\left(R_{2}\right)=0 \text { for } j<n-k .
\end{gathered}
$$

Then the hypotheses of Theorem 3.1 hold with $m=2, r_{k}=0, r_{j}=1$ for $k+1 \leqslant j$ $\leqslant n-k-1$, and $r_{n-k}=2$; we have $r_{n-j}=2-r_{j}$. Consider the resolution (3.18) of $\Theta$ and set $D_{n}=0$. From Lemmas 5.2 and 5.3, we obtain

Proposition 5.1. For $0 \leqslant j \leqslant n-1$, there exists a differential operator

$$
\alpha_{j}: \mathscr{E}^{j} \oplus \mathscr{E}^{n-j-1} \rightarrow \Lambda^{n-1} \mathscr{T}^{*}
$$

of order $r_{j+1}-r_{j}$ such that

$$
\left\langle D_{j} u, v\right\rangle+(-1)^{j}\left\langle u, D_{n-j-1} v\right\rangle=d \alpha_{j}(u \oplus v)
$$

for all $u \in \mathscr{E}^{j}$ and $v \in \mathscr{E}^{n-j-1}$.

Suppose that $X$ is oriented. Consider the mapping sending $u \otimes v$, with $u \in C^{\infty}\left(E^{j}\right)$ and $v \in C_{0}^{\infty}\left(E^{n-j}\right)$ satisfying $D_{j} u=0$ and $D_{n-j} v=0$, onto $\int_{X}\langle u, v\rangle$; by Proposition 5.1 and Stokes' theorem, we see that this mapping induces a well-defined pairing

$$
H^{j}\left(\Gamma\left(\mathscr{E}^{\bullet}\right)\right) \otimes H^{n-j}\left(\Gamma_{0}\left(\mathscr{E}^{\circ}\right)\right) \rightarrow \mathbf{R} .
$$

From the isomorphisms $H_{\Phi}^{i}(X, \Theta) \rightarrow H^{i}\left(\Gamma_{\Phi}\left(\mathscr{E}^{\bullet}\right)\right)$, determined by the resolution (3.18) of $\Theta$, and the pairing (5.13), we obtain a pairing

$$
H^{j}(X, \Theta) \otimes H_{0}^{n-j}(X, \Theta) \rightarrow \mathbf{R} .
$$

It follows directly from the commutativity of diagram (5.9) and the definition of the mappings (3.19) that the diagram

$$
\begin{array}{ccc}
H^{j}\left(\Gamma\left(\mathscr{C}^{0}\right)\right) \otimes H^{n-j}\left(\Gamma_{0}\left(\mathscr{C}^{0}\right)\right) & \rightarrow & \mathbf{R} \\
\downarrow \rho \otimes \rho & & \downarrow \text { id } \\
H^{j}\left(\Gamma\left(\mathscr{E}^{\bullet}\right)\right) \otimes H^{n-j}\left(\Gamma_{0}\left(\mathscr{E}^{\bullet}\right)\right) & \rightarrow & \mathbf{R},
\end{array}
$$

whose horizontal arrows are the mappings (1.21) and (5.13), is commutative. 
The commutativity of diagram (3.20) given by Theorem 3.1 now implies the following

THEOREM 5.1. If $X$ is oriented, the pairings (1.22) and (5.14) are equal.

Thus from Theorem 1.1, if $X$ is oriented and if $R_{2, x}$ is a semisimple Lie algebra for all $x \in X$, we deduce that the pairing (5.14) is nondegenerate.

We continue to assume that there is an integer $0 \leqslant k \leqslant(n+1) / 2$ such that (5.10), (5.11) and (5.12) hold. Suppose moreover that $\operatorname{gr} R_{2, x}$ is a semisimple Lie algebra for all $x \in X$. Let $\tau: g \rightarrow g$ be the involution defined by

$$
\tau(\xi+\eta+\zeta)=-\xi+\eta-\zeta
$$

for $\xi \in g_{0}, \eta \in g_{1}$ and $\zeta \in g_{2}$. Since $g$ is a locally trivial bundle of Lie algebras, according to Lemma 1.3 there exists a morphism of vector bundles $\sigma: g \rightarrow g$ such that $\sigma \tau=\tau \sigma$ and such that $\sigma_{x}$ is a Cartan involution of $g_{x}$ for all $x \in X$. By Propositions 4.1 and 4.2 , we see that

$$
\sigma\left(g_{p}\right)=g_{2-p}
$$

for $p=0,1,2$. Then the mapping $\bar{B}_{\sigma}: g \otimes g \rightarrow \mathbf{R}$, defined by

$$
\bar{B}_{\sigma}(\xi, \eta)=-\bar{B}(\xi, \sigma(\eta))
$$

for $\xi, \eta \in g$, determines a symmetric positive definite form on $g$, that is, a scalar product on the vector bundle $g$. We consider the Riemannian metric on $X$ and the scalar product $(, \quad)$ on $\wedge T^{*} \otimes g$ induced by $\bar{B}_{\sigma}$.

Let $\delta^{*}: \wedge^{j} T^{*} \otimes g_{p} \rightarrow \wedge^{j-1} T^{*} \otimes g_{p+1}$ be the adjoint of $\delta$ and

$$
F^{j}=\left\{u \in \wedge^{j} T^{*} \otimes g_{r_{1}} \mid \delta u=0, \delta^{*} u=0\right\} .
$$

As we have seen in $\S 4$, we have isomorphisms

$$
\gamma: F^{j} \rightarrow E^{j} .
$$

By means of these isomorphisms $\gamma$, from the sequence (3.18) we obtain a resolution

$$
0 \rightarrow \Theta \stackrel{i}{\rightarrow} \mathscr{F}^{0} \stackrel{P^{0}}{\rightarrow} \mathscr{F}^{1} \stackrel{P^{1}}{\rightarrow} \mathscr{F}^{2} \stackrel{P^{2}}{\rightarrow} \cdots \rightarrow \mathscr{F}^{n} \rightarrow 0
$$

of $\Theta$, wheie $F^{0}=E^{0}=T, F^{n}=E^{n}=\wedge^{n} T^{*} \otimes g_{2}$ and $P^{j}$ is a differential operator of order $r_{j+1}-r_{j}+1$. The mappings (4.4), with $\mathfrak{g}=g_{x}$ for $x \in X$, determine morphisms

$$
F^{j} \otimes F^{n-j} \rightarrow \wedge^{n} T^{*},
$$

sending $u \otimes v$ into $\langle u, v\rangle=\langle\gamma u, \gamma v\rangle$, such that

$$
\langle u, v\rangle=(-1)^{n j+j}\langle v, u\rangle
$$

for $u \in F^{j}$ and $v \in F^{n-j}$; according to Proposition 4.4, these morphisms are nondegenerate. From Proposition 5.1, we deduce that for $0 \leqslant j \leqslant n-1$ there exists a differential operator

$$
\tilde{\alpha}_{j}: \mathscr{F}^{j} \oplus \mathscr{F}^{n-j \cdots 1} \rightarrow \wedge^{n-1} \mathscr{T}^{*}
$$

of order $r_{j+1}-r_{j}$ such that

$$
\left\langle P^{j} u, v\right\rangle+(-1)^{j}\left\langle u, P^{n-j-1} v\right\rangle=d \tilde{\alpha}_{j}(u \oplus v)
$$


for all $u \in \mathscr{F}^{j}$ and $v \in \mathscr{F}^{n-j-1}$. We also consider the formal adjoint $P^{j *}: \mathscr{F}^{j} \rightarrow$ $\mathscr{F}^{j-1}$ of $P^{j}$, which depends on $\sigma$.

If $X$ is oriented, let $\Omega$ be the corresponding volume form. As in $\S 4$, let

$$
*: \wedge^{j} T^{*} \otimes g \rightarrow \wedge^{n-j} T^{*} \otimes g
$$

be the mapping defined in terms of the Hodge $*$-isomorphism by

$$
*(\alpha \otimes \xi)=-(* \alpha) \otimes \sigma(\xi)
$$

for $\alpha \in \wedge^{j} T^{*}$ and $\xi \in g$. By restriction, it determines an isomorphism

$$
*: F^{j} \rightarrow F^{n-j}
$$

such that (1.24) and (1.25) hold for all $u, v \in F^{j}$. Then, as in $\S 1$, from the formulas (5.19) and Stokes' theorem, it is easily seen that

$$
P^{j *}=(-1)^{n j+1} * P^{n-j-1} *
$$

(see [7, Proposition 14.1]).

Let $o$ be the orientation sheaf (over $\mathbf{Z}$ ) of $X$ and let $i: \diamond \rightarrow \wedge^{n} \mathscr{T}^{*}$ be the natural mapping determined by the metric on $X$ (see $[7, \S 15])$. If we consider the composition

$$
\Theta \rightarrow \mathscr{T}^{\sigma} \rightarrow g_{2}
$$

the sequence

$$
0 \rightarrow \otimes_{\mathbf{Z}} \Theta \stackrel{i \otimes \sigma}{\rightarrow} \bigwedge^{n} \mathscr{T}^{*} \otimes g_{2} \stackrel{P}{n-1 *}^{\rightarrow} \mathscr{F}^{n-1}
$$

is exact. Indeed, if $\Omega$ is a local volume form on $X$, by (5.22), we have

$$
P^{n-1 *}(\Omega \otimes u)=-* P^{0} *(\Omega \otimes u)=* P^{0} \sigma(u)
$$

for $u \in g_{2}$; hence $P^{n-1 *}(\Omega \otimes u)=0$ if and only if $\sigma(u) \in \Theta$. Therefore by taking local orientations of $X$, from the formulas (5.22) we deduce

Proposition 5.2. The adjoint complex

$$
0 \rightarrow \circ \otimes_{\mathbf{Z}} \Theta \stackrel{i \otimes \sigma}{\rightarrow} \mathscr{F}^{n} \stackrel{P^{n-1 *}}{\rightarrow} \mathscr{F}^{n-1} \stackrel{P^{n-2 *}}{\rightarrow} \mathscr{F}^{n-2} \rightarrow \cdots \rightarrow \mathscr{F}^{1} \stackrel{P^{0 *}}{\rightarrow} \mathscr{F}^{0} \rightarrow 0
$$

of (5.17) is exact.

We introduce the Laplacians $\square^{j}: \mathscr{F}^{j} \rightarrow \mathscr{F}^{j}$ defined by

$$
\square^{j}=\left(P^{j-1} P^{j-1 *}\right)^{r_{j+1}-r_{j}+1}+\left(P^{j *} P^{j}\right)^{r_{j}-r_{j-1}+1},
$$

where $P^{-1}=0$ and $P^{n}=0$. Since (3.18) (with $\Theta$ omitted) is an elliptic complex, so is (5.17); therefore by Lemma 14.2 of [7], these Laplacians are selfadjoint and elliptic, and, if $X$ is compact, the harmonic space

$$
\left\{u \in C^{\infty}\left(F^{j}\right) \mid \square^{j} u=0\right\}=\left\{u \in C^{\infty}\left(F^{j}\right) \mid P^{j-1 *} u=0, P^{j} u=0\right\}
$$

is finite-dimensional and isomorphic to $H^{j}(X, \Theta)$. If $X$ is oriented, from (5.22) it follows that $* \square^{j}=\square^{n-j}$ as differential operators $\mathscr{F}^{j} \rightarrow \mathscr{F}^{n-j}$. As in [7, §14], one may use these facts to prove that the pairings (5.13) and (5.14) are nondegenerate when $X$ is compact and oriented. 
We summarize some of the above results in

THEOREM 5.2. Let $R_{2} \subset J_{2}(T)$ be a formally integrable and formally transitive Lie equation with $g_{3}=0$. Assume that $\pi_{1} R_{2}$ is a sub-bundle of $J_{1}(T)$ and that the mappings (3.2) are of constant rank for all $j \geqslant 0$ and $l=1,2$. Suppose moreover that there is an integer $0 \leqslant k \leqslant(n+1) / 2$ such that (5.10), (5.11) and (5.12) hold and that $g_{x}=\operatorname{gr} R_{2, x}$ is a semisimple Lie algebra for all $x \in X$. Then there exists a morphism $\sigma: g \rightarrow g$ such that $\sigma_{x}$ is a Cartan involution of $g_{x}$ for all $x \in X$, and such that (5.15) holds. Furthermore, in terms of the scalar product on $\wedge T^{*} \otimes g$ induced by $\bar{B}_{\sigma}$, we have a resolution (5.17) of $\Theta$ which is an elliptic complex and which is formally selfadjoint in the following sense:

(i) the relations (5.19) hold for all $u \in \mathscr{F}^{j}$ and $v \in \mathscr{F}^{n-j-1}$;

(ii) if $X$ is oriented, the isomorphisms (5.21) satisfy (1.24) and (1.25), with $u$, $v \in F^{j}$

(iii) if $X$ is oriented, the formal adjoint $P^{j *}$ of $P^{j}$ is given by (5.22);

(iv) the Laplacians $\square^{j}: \mathscr{F}^{j} \rightarrow \mathscr{F}^{j}$ defined by (5.24) are formally selfadjoint and elliptic and, if $X$ is oriented, satisfy the relations

$$
* \square^{j}=\square^{n-j} *: \mathscr{F}^{j} \rightarrow \mathscr{F}^{n-j} .
$$

Remark 5.1. If gr $R_{2, x}$ is a semisimple Lie algebra for all $x \in X$, and if there are integers $r_{j}$ with $0 \leqslant r_{j} \leqslant 2,0 \leqslant j \leqslant n$, such that $H^{p . j}\left(R_{2}\right)=0$ for $p \neq r_{j}$ with $0 \leqslant j \leqslant n, 0 \leqslant p \leqslant 2$, by Proposition 4.4 there exists an integer $0 \leqslant k \leqslant(n+1) / 2$ such that (5.10), (5.11) and (5.12) hold.

6. Riemannian manifolds with constant curvature. Let $R_{1} \subset J_{1}(T)$ be the Killing equation of a Riemannian manifold $(X, g)$ as defined in $\S 3$ of [5]. Let $g^{b}: T \rightarrow T^{*}$ and $g^{\sharp}: T^{*} \rightarrow T$ be the isomorphisms determined by the metric $g$. If $g_{1} \subset T^{*} \otimes T$ is the symbol of $R_{1}$ and $\psi: T^{*} \otimes T \rightarrow S^{2} T^{*}$ is the morphism sending $\alpha \otimes \xi$ into $\alpha \cdot g^{b}(\xi)$, the sequence

$$
0 \rightarrow g_{1} \rightarrow T^{*} \otimes T \stackrel{\psi}{\rightarrow} S^{2} T^{*} \rightarrow 0
$$

is exact. The image of the morphism

$$
\iota: \wedge^{2} T^{*} \rightarrow T^{*} \otimes T
$$

defined by

$$
\iota(\alpha \wedge \beta)=\alpha \otimes g^{\sharp}(\beta)-\beta \otimes g^{\sharp}(\alpha)
$$

for $\alpha, \beta \in T^{*}$, is equal to $g_{1}$. Let $\mu: \wedge^{j} T^{*} \otimes \wedge^{2} T^{*} \rightarrow \wedge^{j+1} T^{*} \otimes T^{*}$ be the morphism of vector bundles defined by

$$
\mu(\omega \otimes(\alpha \wedge \beta))=(\alpha \wedge \omega) \otimes \beta-(\omega \wedge \beta) \otimes \alpha
$$

for $\omega \in \wedge^{j} T^{*}$ and $\alpha, \beta \in T^{*}$, and let $E_{j-1}$ denote its kernel. It is easily verified that the diagram

$$
\begin{array}{ccc}
\wedge^{j+1} T^{*} \otimes \wedge^{2} T^{*} & \stackrel{\mu}{\rightarrow} & \wedge^{j+2} T^{*} \otimes T^{*} \\
\downarrow \text { id } \otimes \iota & & \downarrow \text { id } \otimes g^{*} \\
\wedge^{j+1} T^{*} \otimes g_{1} & \stackrel{\delta}{\rightarrow} & \wedge^{j+2} T^{*} \otimes T
\end{array}
$$


is commutative, and that its mapping $\delta$ is surjective for $j \geqslant 0$. Moreover, for $j=0$, the mappings $\mu$ and $\delta$ of this diagram are isomorphisms; we have

$$
\mu^{-1}\left(\left(\alpha_{1} \wedge \alpha_{2}\right) \otimes \beta\right)=\frac{1}{2}\left(\alpha_{1} \otimes\left(\beta \wedge \alpha_{2}\right)-\alpha_{2} \otimes\left(\beta \wedge \alpha_{1}\right)+\beta \otimes\left(\alpha_{1} \wedge \alpha_{2}\right)\right)
$$

for $\alpha_{1}, \alpha_{2}, \beta \in T^{*}$, and the inverse of $\delta$ will be denoted by $\lambda$. The image of the morphism of vector bundles

$$
\tau: S^{2} T^{*} \otimes S^{2} T^{*} \rightarrow \wedge^{2} T^{*} \otimes \wedge^{2} T^{*},
$$

determined by

$$
\begin{aligned}
\tau\left(\left(\alpha_{1} \cdot \alpha_{2}\right) \otimes\left(\beta_{1} \cdot \beta_{2}\right)\right)=\frac{1}{2}\left\{\left(\alpha_{1} \wedge \beta_{1}\right) \otimes\left(\alpha_{2} \wedge \beta_{2}\right)+\left(\alpha_{1} \wedge \beta_{2}\right) \otimes\left(\alpha_{2} \wedge \beta_{1}\right)\right. \\
\left.+\left(\alpha_{2} \wedge \beta_{1}\right) \otimes\left(\alpha_{1} \wedge \beta_{2}\right)+\left(\alpha_{2} \wedge \beta_{2}\right) \otimes\left(\alpha_{1} \wedge \beta_{1}\right)\right\}
\end{aligned}
$$

for $\alpha_{1}, \alpha_{2}, \beta_{1}, \beta_{2} \in T^{*}$, is equal to $E_{1}$ (see [5, §3]).

Lemma 6.1. For $u \in T^{*} \otimes T$ and $\alpha_{1}, \alpha_{2} \in T^{*}$, we have

$($ id $\otimes \iota) \tau\left(\alpha_{1} \cdot \alpha_{2} \otimes(\psi u)\right)=\alpha_{1} \wedge \lambda\left(\alpha_{2} \wedge u\right)+\alpha_{2} \wedge \lambda\left(\alpha_{1} \wedge u\right)$

as elements of $\wedge^{2} T^{*} \otimes g_{1}$.

Proof. Assume that $u=\alpha \otimes \xi$, with $\alpha \in T^{*}$ and $\xi \in T$, and set $\beta=g^{b}(\xi)$. By the commutativity of (6.2) with $j=0$, we have

$$
\begin{aligned}
\left(\mathrm{id} \otimes \iota^{-1}\right)\left(\alpha_{1} \wedge\right. & \left.\lambda\left(\alpha_{2} \wedge u\right)+\alpha_{2} \wedge \lambda\left(\alpha_{1} \wedge u\right)\right) \\
= & \left(\mathrm{id} \otimes \iota^{-1}\right)\left(\alpha_{1} \wedge \lambda\left(\left(\alpha_{2} \wedge \alpha\right) \otimes \xi\right)+\alpha_{2} \wedge \lambda\left(\left(\alpha_{1} \wedge \alpha\right) \otimes \xi\right)\right) \\
& =\alpha_{1} \wedge \mu^{-1}\left(\left(\alpha_{2} \wedge \alpha\right) \otimes \beta\right)+\alpha_{2} \wedge \mu^{-1}\left(\left(\alpha_{1} \wedge \alpha\right) \otimes \beta\right) \\
& =\tau\left(\left(\alpha_{1} \cdot \alpha_{2}\right) \otimes(\alpha \cdot \beta)\right)=\tau\left(\left(\alpha_{1} \cdot \alpha_{2}\right) \otimes(\psi u)\right) .
\end{aligned}
$$

We now assume that $(X, g)$ has constant curvature. Then according to Theorem 6.1 of [5], the equation $R_{1}$ is formally integrable. It is also a formally transitive Lie equation with $g_{2}=0$ (see [5, §3]). Thus this equation satisfies the assumptions of $\S 1$ and so we may consider the sequence (1.14) with $m=1$, where $\Theta$ is the sheaf of Killing vector fields of $(X, g)$.

From the exactness of the sequence (6.1), we see that the mapping $\psi$ induces an isomorphism

$$
H^{0,1}\left(R_{1}\right) \rightarrow S^{2} T^{*}
$$

Since the mapping $\delta$ of (6.2) is surjective, we have $H^{0, j}\left(R_{1}\right)=0$ for $j \geqslant 2$; the isomorphism id $\otimes \imath$ of this diagram induces by restriction an isomorphism of vector bundles

$$
\text { id } \otimes \imath: E_{j} \rightarrow H^{1, j+1}\left(g_{1}\right) \text { for } j \geqslant 0 .
$$

Clearly

$$
H^{1,0}\left(g_{1}\right)=H^{1,1}\left(g_{1}\right)=0 \text {. }
$$

By the above computation of the Spencer cohomology groups of $R_{1}$, we see that the hypotheses of Theorem 3.1 are satisfied with $r_{n}=r_{1}=0, r_{2}=\cdots=r_{n}=1$, and so we have a resolution (3.18)

$$
0 \rightarrow \Theta \rightarrow \mathscr{T}^{D_{0}} \mathscr{H}^{0,1}\left(R_{1}\right) \stackrel{D_{1}}{\rightarrow} \mathscr{H}^{1,2}\left(g_{1}\right) \stackrel{D_{2}}{\rightarrow} \cdots \rightarrow \mathscr{H}^{1, n}\left(g_{1}\right) \rightarrow 0
$$


of $\Theta$, where $D_{0}, D_{j}$ (with $j \geqslant 2$ ) are differential operators of order 1 and $D_{1}$ is of order 2. By means of the isomorphisms (6.3) and (6.4), we obtain a resolution

$$
0 \rightarrow \Theta \rightarrow \mathscr{T}^{\tilde{D}_{0}} \rightarrow S^{2} \mathscr{T}^{*} \stackrel{\tilde{D}_{1}}{\rightarrow} \mathscr{E}_{1} \stackrel{\tilde{D}_{2}}{\rightarrow} \mathscr{E}_{2} \rightarrow \cdots \rightarrow \mathscr{E}_{n-1} \rightarrow 0
$$

of $\Theta$. We claim that this sequence is the Calabi sequence of [2] as defined in [5]. Let

$$
Q_{0}: \mathscr{T} \rightarrow S^{2} \mathscr{T}^{*}, \quad Q_{1}: S^{2} \mathscr{T}^{*} \rightarrow \mathscr{E}_{1}, \quad Q_{j}: \mathscr{E}_{j-1} \rightarrow \mathscr{E}_{j} \quad \text { for } 2 \leqslant j \leqslant n-2
$$

be the differential operators of the Calabi sequence which are defined as follows. For $\xi \in \mathscr{T}$, we have $Q_{0} \xi=\mathscr{L}_{\xi}$ g, where $\mathscr{L}_{\xi}$ g denotes the Lie derivative along the vector field $\xi$ of the metric $g$. According to [5, §3], the symbol of $Q_{0}$ is equal to $\psi$. If $\overline{\mathscr{R}}_{g}^{\prime}$ is the linearization along $g$ of the curvature operator sending a metric into its curvature of type $(2,2)$, which is a section of $\wedge^{2} T^{*} \otimes \wedge^{2} T$, and if $g^{b}: \wedge^{2} T \rightarrow \wedge^{2} T^{*}$ is the isomorphism given by $g$, we consider the differential operator of order 2

$$
D_{g}: S^{2} \mathscr{T}^{*} \rightarrow \wedge^{2} \mathscr{T}^{*} \otimes \wedge^{2} \mathscr{T}^{*}
$$

sending $h$ into $g^{\text {b }} \circ \overline{\mathscr{R}}_{g}^{\prime}(h)$. Then $D_{g}\left(S^{2} \mathscr{T}^{*}\right) \subset \mathscr{E}_{1}$ and we set $Q_{1}=D_{g}: S^{2} \mathscr{T}^{*} \rightarrow \mathscr{E}_{1}$. According to equation (4.28) and Proposition 6.1 of [5], the symbol of $Q_{1}$ is equal to $\tau$. Let $\tilde{\nabla}$ be the Levi-Civita connection of $g$ and the connection in $\wedge^{k} T^{*}$ induced by $\tilde{\nabla}$. This connection determines a differential operator

$$
\tilde{\nabla}^{\wedge}: \wedge^{j \mathscr{T}^{*}} \otimes \wedge^{k} \mathscr{T}^{*} \rightarrow \wedge^{j+1} \mathscr{T}^{*} \otimes \wedge^{k} \mathscr{T}^{*}
$$

satisfying

$$
\tilde{\nabla}^{\wedge}(\omega \otimes \alpha)=d \omega \otimes \alpha+(-1)^{j} \omega \wedge \tilde{\nabla} \alpha
$$

for $\omega \in \wedge^{j} \mathscr{T}^{*}$ and $\alpha \in \Lambda^{k} \mathscr{T}^{*}$. We consider this operator with $j \geqslant 2$ and $k=2$. According to [5, §6], we have $\tilde{\nabla}^{\wedge}\left(\mathscr{E}_{j-1}\right) \subset \mathscr{E}_{j}$ for $j \geqslant 2$, and we set

$$
Q_{j}=\tilde{\nabla}^{\wedge}: \mathscr{E}_{j-1} \rightarrow \mathscr{E}_{j} \text { for } j \geqslant 2 .
$$

The symbol

$$
\sigma(\tilde{\nabla}): T^{*} \otimes \wedge^{j} T^{*} \otimes \wedge^{2} T^{*} \rightarrow \wedge^{j+1} T^{*} \otimes \wedge^{2} T^{*}
$$

of (6.6), with $k=2$, sends $\alpha \otimes \omega \otimes \beta$ into $\alpha \wedge \omega \otimes \beta$ for $\alpha \in T^{*}, \omega \in \wedge^{j} T^{*}$ and $\beta \in \wedge^{2} T^{*}$. In [5, §3], for $j \geqslant 2$ it is shown that the symbol

$$
\sigma\left(Q_{j}\right): T^{*} \otimes E_{j-1} \rightarrow E_{j},
$$

which is the restriction of (6.7), is an epimorphism of vector bundles.

We first verify that $\sigma\left(\tilde{D}_{j}\right)=\sigma\left(Q_{j}\right)$ for $j \geqslant 0$. According to the definition of $\tilde{D}_{0}$, the diagram

$$
\begin{array}{ccc}
\mathscr{R}_{1} & \stackrel{\nabla}{\rightarrow} & \mathscr{T}^{*} \otimes \mathscr{R}_{1} \\
\downarrow \pi_{0} & & \downarrow \psi \cdot \pi_{0} \\
\mathscr{T} & \stackrel{\tilde{D}_{0}}{\rightarrow} & S^{2} \mathscr{T}^{*}
\end{array}
$$

commutes; since the symbol of $\nabla$ is equal to the identity mapping of $T^{*} \otimes R_{1}$, the symbol of $\tilde{D}_{0}$ is equal to $\psi$, and so $\sigma\left(\tilde{D}_{0}\right)=\sigma\left(Q_{0}\right)$. Let $Q: \mathscr{T}^{*} \otimes \mathscr{R}_{1} \rightarrow \mathscr{H}^{1.2}\left(g_{1}\right)$ be 
the differential operator defined by

$$
Q u=\nabla^{\wedge}\left(u+\lambda \pi_{0} \nabla^{\wedge} u\right)
$$

for $u \in \mathscr{T}^{*} \otimes \mathscr{R}_{1}$. The commutativity of diagram (3.10) tells us that the diagram

$$
\begin{array}{ccc}
\mathscr{T}^{*} \otimes \mathscr{R}_{1} & \stackrel{Q}{\rightarrow} & \mathscr{H}^{1.2}\left(g_{1}\right) \\
\downarrow \rho_{0} & \nearrow D_{1} & \\
\mathscr{H}^{0.1}\left(R_{1}\right) &
\end{array}
$$

commutes, and by the definition of $\tilde{D}_{1}$ that

$$
\begin{array}{ccc}
\mathscr{T}^{*} \otimes \mathscr{R}_{1} & \stackrel{Q}{\rightarrow} & \mathscr{H}^{1,2}\left(g_{1}\right) \\
\downarrow \psi \cdot \pi_{0} & & \uparrow \text { id } \otimes \iota \\
S^{2} \mathscr{T}^{*} & \stackrel{\tilde{D}_{1}}{\rightarrow} & \mathscr{E}_{1}
\end{array}
$$

commutes. According to (3.11) and Lemma 6.1, we see that $\sigma\left(\tilde{D}_{1}\right)=\tau=\sigma\left(Q_{1}\right)$. Clearly, we have $\sigma\left(\tilde{D}_{j}\right)=\sigma\left(Q_{j}\right)$ for $j \geqslant 2$.

Thus we know that $\tilde{D}_{j}-Q_{j}$ is a differential operator of order 0 for $j \neq 1$, and that $\tilde{D}_{1}-Q_{1}$ is of order 1 . We now show that $\tilde{D}_{j}=Q_{j}$ for $j \geqslant 0$. Since $\tilde{D}_{0}-Q_{0}$ vanishes on $\Theta$ and since for all $x \in X$ and $\eta \in T_{x}$ there exists a Killing vector field $\xi$ on a neighborhood of $x$ such that $\xi(x)=\eta$, we see that $\tilde{D}_{0}=Q_{0}$. If $x \in X$ and $h \in S^{2} \mathscr{T}_{x}^{*}$, by Lemma 5.1 of [5], there exists $\xi \in \mathscr{T}_{x}$ such that $j_{1}\left(\mathscr{L}_{\xi} g\right)(x)=j_{1}(h)(x)$; hence we see that

$$
\left(\left(\tilde{D}_{1}-Q_{1}\right) h\right)(x)=\left(\left(\tilde{D}_{1}-Q_{1}\right) Q_{0} \xi\right)(x)=0,
$$

and that $\tilde{D}_{1}=Q_{1}$. Since

$$
\sigma\left(Q_{1}\right)=\tau: S^{2} T^{*} \otimes S^{2} T^{*} \rightarrow E_{1}, \quad \sigma\left(Q_{j}\right): T^{*} \otimes E_{j} \rightarrow E_{j+1},
$$

for $j \geqslant 1$, are epimorphisms, if $x \in X$ and $u \in \mathscr{E}_{j, x}$ with $j \geqslant 1$, there exists $v \in S^{2} \mathscr{T}_{x}^{*}$ if $j=1$, or $v \in \mathscr{E}_{j-1, x}$ if $j \geqslant 2$ such that $\left(Q_{j-1} v\right)(x)=u(x)$; then since (6.5) and the Calabi sequence are complexes, if $\tilde{D}_{j-1}=Q_{j-1}$ with $j \geqslant 2$, we have

$$
\left(\left(\tilde{D}_{j}-Q_{j}\right) u\right)(x)=\left(\left(\tilde{D}_{j}-Q_{j}\right) \cdot Q_{j-1} v\right)(x)=0,
$$

and so $\tilde{D}_{j}=Q_{j}$. Thus (6.5) is the Calabi sequence, and now from Theorem 3.1 we obtain the exactness of this sequence.

7. Conformally flat Riemannian manifolds. Let $(X, g)$ be a conformally flat Riemannian manifold of dimension $n \geqslant 3$. Let $R_{1} \subset J_{1}(T)$ be the conformal Killing equation of $(X, g)$ as defined in $\S 2$ of [7] whose solutions are the conformal Killing vector fields. According to Propositions 5.1 and 6.1 of [7], the equation $R_{1}$ is formally integrable. It is also a formally transitive Lie equation with $g_{3}=0$, and so $R_{1}$ satisfies the assumptions of $\S 1$ with $k=1$ and $m=2$. Therefore we may consider the sequence (1.14) with $m=2$, where $\Theta$ is the sheaf of conformal Killing vector fields of $(X, g)$. According to $\S \S 2$ and 10 of [7], we know that $R_{2}$ satisfies the hypotheses of Theorem 3.1 with $m=2, r_{1}=0, r_{j}=1$ for $2 \leqslant j \leqslant n-2$, and 
$r_{n-1}=2$; in particular (5.10), (5.11) and (5.12) hold with $k=1$. Therefore, we also have the resolution (3.18) of $\Theta$, where

$$
\begin{aligned}
& E^{0}=T, \quad E^{1}=H^{0,1}\left(R_{1}\right), \\
& E^{j}=H^{1, j}\left(g_{1}\right) \quad \text { for } 2 \leqslant j \leqslant n-2, \\
& E^{n-1}=H^{2, n-1}\left(g_{1}\right), \quad E^{n}=H^{2, n}\left(g_{1}\right)=\wedge^{n} T^{*} \otimes g_{2} .
\end{aligned}
$$

If id is the section of $T^{*} \otimes T=\operatorname{Hom}(T, T)$ which corresponds to the identity mapping of $T$, and if $\{\mathrm{id}\}$ is the sub-bundle of $T^{*} \otimes T$ generated by this section, then $g_{1}=\bar{g}_{1} \oplus\{$ id $\}$, where $\bar{g}_{1}$ denotes the kernel of the morphism $\psi$ of $\S 6$. According to Lemma 2.1 and formula (2.2) of [7], the image of the monomorphism of vector bundles

$$
\bar{\theta}: T^{*} \rightarrow S^{2} T^{*} \otimes T
$$

defined by

$$
\bar{\theta}(\alpha)(\xi, \eta)=\langle\xi, \alpha\rangle \eta+\langle\eta, \alpha\rangle \xi-g(\xi, \eta) g^{\sharp}(\alpha)
$$

for $\alpha \in T^{*}$ and $\xi, \eta \in T$, is equal to $g_{2}$; in fact, the morphism $\hat{\theta}$ of [7, §2] is equal to $\frac{1}{2} \bar{\theta}$. Using (5.2) and (5.4), we easily verify that

$$
\begin{gathered}
{[\xi, \bar{\theta}(\alpha)]=\langle\xi, \alpha\rangle \mathrm{id}+\alpha \otimes \xi-g^{b}(\xi) \otimes g^{\sharp}(\alpha),} \\
{[\bar{\theta}(\alpha), u]=-\bar{\theta}\left(u^{*}(\alpha)\right)}
\end{gathered}
$$

as elements of $g$ for $\alpha \in T^{*}, \xi \in T$ and $u \in g_{1}$, where $u^{*} \in \operatorname{Hom}\left(T^{*}, T^{*}\right)$ is the adjoint of $u \in \operatorname{Hom}(T, T)$. By means of these formulas, (5.2) and (5.3), we easily see that the Killing form $\bar{B}$ of $g$ is given by

$$
\begin{gathered}
\bar{B}(\bar{\theta}(\alpha), \xi)=-2 n\langle\xi, \alpha\rangle, \\
\bar{B}(u, v)=n \operatorname{Tr}(u \circ v)
\end{gathered}
$$

for $\alpha \in T^{*}, \xi \in T$ and $u, v \in \bar{g}_{1}$, and

$$
\bar{B}(\text { id, id })=2 n, \quad \bar{B}\left(\text { id, } \bar{g}_{1}\right)=0, \quad \bar{B}_{1 g_{2}}=0, \quad \bar{B}_{\mid g_{0}}=0,
$$

and that $g_{1}$ and $g_{0} \oplus g_{2}$ are perpendicular to each other with respect to $\bar{B}$. It follows that $\bar{B}$ is nondegenerate and so gr $R_{2, x}$ is a semisimple Lie algebra for all $x \in X$. In fact, since the natural representation of $g_{1, x}$ on $T_{x}$ is irreducible and $g_{2, x} \neq 0$, by Proposition 4.3 the Lie algebra gr $R_{2, x}$ is simple for $x \in X$. It can be shown that gr $R_{2, x}$ is isomorphic to $\mathfrak{5 0}(n+1,1)$ and that $R_{2, x}$ is isomorphic to $\operatorname{gr} R_{2, x}$ (see $[11,12])$. The assertion of Proposition 4.4 for gr $R_{2, x}$ is given by [7, Proposition 10.1].

Let $S_{0}^{2} T^{*}$ be the sub-bundle of $S^{2} T^{*}$ of symmetric 2-tensors with zero trace and, as in $\S 8$ of [7], let $E_{j}^{0}$ denote the sub-bundle of $E_{j}$ consisting of those elements with zero trace (with respect to $g$ ). As in [7, §11], we set

$$
\begin{aligned}
& F_{0}=T, \quad F_{1}=S_{0}^{2} T^{*}, \\
& F_{j}=E_{j-1}^{0} \quad \text { for } 2 \leqslant j \leqslant n-2, \\
& F_{n-1}=\wedge^{n-1} T^{*} \otimes S_{0}^{2} T^{*}, \quad F_{n}=\wedge^{n} T^{*} \otimes T^{*} .
\end{aligned}
$$

We now define isomorphisms $\varphi_{j}: F_{j} \rightarrow E^{j}$ for $0 \leqslant j \leqslant n$. 
Let $\varphi_{0}$ be the identity mapping of $T$. Let $\nu: S^{2} T^{*} \rightarrow T^{*} \otimes T$ be the monomorphism of vector bundles determined by

$$
\nu(\alpha \cdot \beta)=\alpha \otimes g^{\sharp}(\beta)+\beta \otimes g^{\sharp}(\alpha)
$$

for $\alpha, \beta \in T^{*}$. Let $p_{0}: S^{2} T^{*} \rightarrow S_{0}^{2} T^{*}$ be the morphism sending $h$ into $h-\frac{1}{n}(\operatorname{Tr} h) g$, where $\operatorname{Tr} h$ is the trace of $h$. Then it is easily verified that the diagram

$$
\begin{aligned}
& T^{*} \otimes T \stackrel{p_{0} \psi}{\rightarrow} \quad S_{0}^{2} T^{*} \stackrel{\frac{1}{2} \nu}{\rightarrow} \quad T^{*} \otimes T \\
& \beta \searrow \quad \measuredangle \beta \\
& E^{1}
\end{aligned}
$$

commutes, where the mapping $\beta$ is the natural projection. Let $\varphi_{1}: S_{0}^{2} T^{*} \rightarrow E^{1}$ be the mapping $\beta \circ \nu$.

Consider the monomorphism of vector bundles

$$
(\mathrm{id} \otimes \iota): \wedge^{j} T^{*} \otimes \wedge^{2} T^{*} \rightarrow \wedge^{j} T^{*} \otimes g_{1}
$$

and its restriction to $E_{j-1}$. For $j \geqslant 2$, according to the commutativity of diagram (6.2), the mapping

$$
\varpi: E_{j-1} \rightarrow H^{1, j}\left(g_{1}\right)
$$

sending $u$ into the cohomology class of (id $\otimes \imath$ ) $u$ is well-defined. According to [7, $\S 10]$, for $2 \leqslant j \leqslant n-2$ its restriction

$$
\varpi: E_{j-1}^{0} \rightarrow H^{1, j}\left(g_{1}\right)
$$

to $E_{j-1}^{0}$ is an isomorphism; moreover, if $\tilde{\rho}_{j-1}: E_{j-1} \rightarrow E_{j-1}^{0}$ denotes the orthogonal projection of $E_{j-1}$ onto $E_{j-1}^{0}$ with respect to the scalar product on $\wedge^{j} T^{*} \otimes \wedge^{2} T^{*}$ induced by $g$, then for $u \in E_{j-1}$ we know that $\tilde{\rho}_{j-1} u$ is the unique element of $E_{j-1}^{0}$ such that

$$
\varpi \tilde{\rho}_{j-1} u=\varpi u .
$$

Let $\varphi_{j}: E_{j-1}^{0} \rightarrow E^{j}$ be equal to $2 \varpi$ for $2 \leqslant j \leqslant n-2$.

If

$$
\operatorname{Tr}^{n-1}: \wedge^{n} T^{*} \otimes S^{2} T^{*} \rightarrow \wedge^{n-1} T^{*} \otimes T^{*}
$$

is the trace mapping determined by $g$ as defined in $\S 8$ of [7], according to $\S \S 2$ or 8 of [7], the restriction of the morphism

$$
(\text { id } \otimes \bar{\theta}) \cdot \operatorname{Tr}^{n-1}: \wedge^{n} T^{*} \otimes S^{2} T^{*} \rightarrow \wedge^{n-1} T^{*} \otimes g_{2}
$$

to $\wedge^{n} T^{*} \otimes S_{0}^{2} T^{*}$ is an isomorphism onto $H^{2, n-1}\left(g_{1}\right)$. Let

$$
\varphi_{n-1}: \wedge^{n} T^{*} \otimes S_{0}^{2} T^{*} \rightarrow F^{n-1}
$$

be equal to $4(\mathrm{id} \otimes \bar{\theta}) \cdot \operatorname{Tr}^{n-1}$ if $n \geqslant 4$, and to (id $\left.\otimes \bar{\theta}\right) \cdot \operatorname{Tr}^{2}$ if $n=3$. Finally, let

$$
\varphi_{n}: \wedge^{n} T^{*} \otimes T^{*} \rightarrow \wedge^{n} T^{*} \otimes g_{2}
$$

be equal to $4($ id $\otimes \bar{\theta})$ if $n \geqslant 4$, and to id $\otimes \bar{\theta}$ if $n=3$.

Let

$$
S^{2} T^{*} \otimes\left(\wedge^{n} T^{*} \otimes S^{2} T^{*}\right) \rightarrow \wedge^{n} T^{*}
$$


be the morphism, sending $h \otimes v$ into $\langle h, v\rangle$, where $h \in S^{2} T^{*}$ and $v \in \Lambda^{n} T^{*} \otimes S^{2} T^{*}$, determined by

$$
\left\langle h, \omega \otimes h^{\prime}\right\rangle=\left(h, h^{\prime}\right) \omega
$$

for $h, h^{\prime} \in S^{2} T^{*}$ and $\omega \in \Lambda^{n} T^{*}$, where $\left(h, h^{\prime}\right)$ is the scalar product of $h$ and $h^{\prime}$ determined by the metric $g($ as in $[7, \S 8])$.

Lemma 7.1. For $h \in S^{2} T^{*}$ and $v \in \Lambda^{n} T^{*} \otimes S^{2} T^{*}$, we have

$$
\left\langle\nu(h),(\mathrm{id} \otimes \bar{\theta}) \operatorname{Tr}^{n-1} v\right\rangle=-2 n\langle h, v\rangle,
$$

where the left-hand side is given by the mapping (4.4).

Proof. Let $x \in X$ and let $\left\{\xi_{1}, \ldots, \xi_{n}\right\}$ be an orthonormal basis for $T_{x}$ (with respect to the metric $g$ ). Let $h=\alpha \cdot \beta \in S^{2} T_{x}^{*}$ with $\alpha, \beta \in T_{x}^{*}$, and $v=\omega \otimes h^{\prime} \in$ $\left(\wedge^{n} T^{*} \otimes S^{2} T^{*}\right)_{x}$, where $\omega \in \wedge^{n} T_{x}^{*}$ and $h^{\prime} \in S^{2} T_{x}^{*}$. We have

$$
0=i\left(\xi_{j}\right)(\alpha \wedge \omega)=\left\langle\xi_{j}, \alpha\right\rangle \omega-\alpha \wedge i\left(\xi_{j}\right) \omega .
$$

Thus by (7.3), we obtain

$$
\begin{aligned}
& \left\langle\nu(h),(\mathrm{id} \otimes \bar{\theta}) \operatorname{Tr}^{n-1} v\right\rangle=\sum_{j=1}^{n}\left\langle\alpha \otimes g^{\sharp}(\beta)+\beta \otimes g^{\sharp}(\alpha),\left(i\left(\xi_{j}\right) \omega\right) \otimes \bar{\theta}\left(i\left(\xi_{j}\right) h^{\prime}\right)\right\rangle \\
& =\sum_{j=1}^{n}\left(\bar{B}\left(g^{\sharp}(\beta), \bar{\theta}\left(i\left(\xi_{j}\right) h^{\prime}\right)\right) \alpha \wedge i\left(\xi_{j}\right) \omega+\bar{B}\left(g^{\sharp}(\alpha), \bar{\theta}\left(i\left(\xi_{j}\right) h^{\prime}\right)\right) \beta \wedge i\left(\xi_{j}\right) \omega\right) \\
& =-2 n\left(\sum_{j=1}^{n}\left(\left\langle g^{\sharp}(\beta), i\left(\xi_{j}\right) h^{\prime}\right\rangle\left\langle\xi_{j}, \alpha\right\rangle+\left\langle g^{\sharp}(\alpha), i\left(\xi_{j}\right) h^{\prime}\right\rangle\left\langle\xi_{j}, \beta\right\rangle\right)\right) \omega \\
& =-2 n\left(\sum_{j=1}^{n}\left(h^{\prime}\left(\xi_{j}, g^{\sharp}(\beta)\right)\left\langle\xi_{j}, \alpha\right\rangle+h^{\prime}\left(\xi_{j}, g^{\sharp}(\alpha)\right)\left\langle\xi_{j}, \beta\right\rangle\right)\right) \omega \\
& =-2 n\left(\sum_{i, j=1}^{n}\left(\left\langle\xi_{j}, \alpha\right\rangle\left\langle\xi_{i}, \beta\right\rangle+\left\langle\xi_{j}, \beta\right\rangle\left\langle\xi_{i}, \alpha\right\rangle\right) h^{\prime}\left(\xi_{j}, \xi_{i}\right)\right) \omega \\
& =-2 n\left(\sum_{i, j=1}^{n} h\left(\xi_{j}, \xi_{i}\right) h^{\prime}\left(\xi_{j}, \xi_{i}\right)\right) \omega=-2 n\left\langle h, \omega \otimes h^{\prime}\right\rangle .
\end{aligned}
$$

If $u, v \in \wedge^{2} T^{*}$, we have

$$
\operatorname{Tr}(\iota(u) \circ \iota(v))=-2(u, v),
$$

where $(, \quad)$ is the scalar product on $\wedge^{2} T^{*}$; thus by (7.4), we see that

$$
\bar{B}(\iota(u), \iota(v))=-2 n(u, v)
$$

for $u, v \in \wedge^{2} T^{*}$. Using this identity, Lemma 7.1 and (7.3), we easily verify

LEMMA 7.2. If $u \in F_{j}$ and $v \in F_{n-j}$, we have

$$
\left\langle\varphi_{j} u, \varphi_{n-j} v\right\rangle=-c n\langle u, v\rangle,
$$

where $\left\langle\varphi_{j} u, \varphi_{n-j} v\right\rangle$ is given by $\S 5$ and $\langle u, v\rangle$ by the mapping (11.33) of [7], and where $c=8$ if $n \geqslant 4$, and $c=2$ if $n=3$. 
We next recall the definition of the operators of the complex (12.5) of [7], namely

$$
0 \rightarrow \Theta \stackrel{i}{\rightarrow} \mathscr{F}_{0} \stackrel{P_{0}}{\rightarrow} \mathscr{F}_{1} \stackrel{P_{1}}{\rightarrow} \mathscr{F}_{2} \stackrel{P_{2}}{\rightarrow} \cdots \rightarrow \mathscr{F}_{n-1} \stackrel{P_{n-1}}{\rightarrow} \mathscr{F}_{n} \rightarrow 0
$$

We have

$$
\begin{array}{ll}
P_{0} \xi=\frac{1}{2} p_{0} \mathscr{L}_{\xi} g & \text { for } \xi \in \mathscr{T}, \\
P_{n-1} v=\tilde{\nabla}^{\wedge} \cdot \operatorname{Tr}^{n-1} v & \text { for } v \in \Lambda^{n} \mathscr{T}^{*} \otimes S_{0}^{2} \mathscr{T}^{*},
\end{array}
$$

where $\tilde{\nabla}^{\wedge}$ is the differential operator (6.6), with $j=n-1$ and $k=1$, determined by the Levi-Civita connection $\tilde{\nabla}$ of $g$. Then $\sigma\left(P_{0}\right)=\frac{1}{2} p_{0} \psi$. If $n \geqslant 4$, the differential operator $P_{1}: \mathscr{F}_{1} \rightarrow \mathscr{F}_{2}$ of order 2 is equal to $\mathscr{W}_{g}^{\prime}$, the linearization along $g$ of the operator sending a metric into its Weyl curvature tensor, which is a section of $E_{1}$; since $(X, g)$ is conformally flat, we have $\mathscr{W}_{g}^{\prime}\left(S_{0}^{2} \mathscr{T}^{*}\right) \subset \mathscr{E}_{1}^{0}$ (see $\left.[7, \S 3]\right)$. From formula (3.18) of [7] and Proposition 8.2 of [7], we deduce that

$$
\sigma\left(P_{1}\right)=\tilde{\rho}_{1} \tau \text {. }
$$

If $n \geqslant 4$ and $2 \leqslant j \leqslant n-3$, the operator $P_{j}$ is given by

$$
P_{j} u=\tilde{\rho}_{j} \tilde{\nabla}^{\wedge} u
$$

for $u \in \mathscr{E}_{j-1}^{0}$, where $\tilde{\nabla}^{\wedge}$ is the differential operator (6.6), with $k=2$, determined by $\tilde{\nabla}$, which satisfies $\tilde{\nabla}^{\wedge}\left(\mathscr{E}_{j-1}\right) \subset \mathscr{E}_{j}$. The symbol $\sigma\left(P_{j}\right)$ is equal to $\tilde{\rho}_{j} \sigma\left(\tilde{\nabla}^{\wedge}\right)$. Let

$$
\hat{\phi}: \wedge^{n-2} T^{*} \otimes T^{*} \rightarrow \wedge^{n-1} T^{*} \otimes \wedge^{2} T^{*}
$$

be the isomorphism of vector bundles defined in $\$ 2$ of [7]; then its restriction

$$
\hat{\phi}: \delta\left(\wedge^{n-3} T^{*} \otimes S^{2} T^{*}\right) \rightarrow E_{n-2}
$$

is an isomorphism (see [7, $\S 2,8])$. Let $R^{0}$ be the section of $T^{*} \otimes T$ determined by

$$
g\left(R^{0}(\xi), \eta\right)=\operatorname{Ric}(\xi, \eta)
$$

where Ric is the Ricci curvature of $(X, g)$ and $\xi, \eta \in T$. We define a morphism

$$
\theta: \wedge^{n-2} T^{*} \otimes \wedge^{2} T^{*} \rightarrow \wedge^{n-1} T^{*} \otimes T^{*}
$$

by

$$
\boldsymbol{\theta}(u)\left(\xi_{1}, \ldots, \xi_{n-1}, \eta\right)=\frac{1}{n-2} \sum_{l=1}^{n-1}(-1)^{l+n-1} u\left(\xi_{1}, \ldots, \hat{\xi}_{l}, \ldots, \xi_{n-1}, R^{0}\left(\xi_{l}\right), \eta\right)
$$

for $u \in \wedge^{n-2} T^{*} \otimes \wedge^{2} T^{*}$ and $\xi_{1}, \ldots, \xi_{n-1}, \eta \in T$. Then there exists a unique differential operator of order 2

$$
P_{n-2}: \mathscr{E}_{n-3}^{0} \rightarrow \bigwedge^{n} \mathscr{T}^{*} \otimes S_{0}^{2} \mathscr{T}^{*}
$$

such that

$$
\operatorname{Tr}^{n-1} \cdot P_{n-2}=\frac{(-1)^{n}}{2}\left(\tilde{\nabla} \wedge \hat{\phi}^{-1} \tilde{\nabla}^{\wedge}-\theta\right): \mathscr{E}_{n-3}^{0} \rightarrow \wedge^{n-1} \mathscr{T}^{*} \otimes \mathscr{T}^{*},
$$

where $\tilde{\nabla}^{\wedge}$ is the differential operator (6.6), with $k=1$ or 2 . Then we see that

$$
\begin{aligned}
\operatorname{Tr}^{n-1} \sigma\left(P_{n-2}\right)\left(\alpha_{1} \cdot \alpha_{2} \otimes u\right) \\
\quad=\frac{(-1)^{n}}{2}\left(\alpha_{1} \wedge \hat{\phi}^{-1}\left(\alpha_{2} \wedge u\right)+\alpha_{2} \wedge \hat{\phi}^{-1}\left(\alpha_{1} \wedge u\right)\right)
\end{aligned}
$$

for $\alpha_{1}, \alpha_{2} \in T^{*}$ and $u \in E_{n-3}^{0}$. 
If $n=3$, let

$$
P_{1}: S_{0}^{2 \mathscr{T}^{*}} \rightarrow \wedge^{3} \mathscr{T}^{*} \otimes S_{0}^{2} \mathscr{T}^{*}
$$

be the differential operator of order 3 defined in $\S 11$ of [7] such that

$$
\operatorname{Tr}^{2} \cdot P_{1}=-\delta(\mathscr{D} \omega)_{g}^{\prime} .
$$

By formula (6.7) of [7], we see that the symbol of $P_{1}$ is determined by the relation (7.13) $\operatorname{Tr}^{2} \cdot \sigma\left(P_{1}\right)\left(\alpha_{1} \cdot \alpha_{2} \cdot \alpha_{3} \otimes u\right)$

$$
\begin{aligned}
=-2\left(\alpha_{1} \wedge \hat{\phi}^{-1} \tau\left(\alpha_{2} \cdot \alpha_{3} \otimes u\right)+\alpha_{2} \wedge\right. & \hat{\phi}^{-1} \tau\left(\alpha_{1} \cdot \alpha_{3} \otimes u\right) \\
& \left.+\alpha_{3} \wedge \hat{\phi}^{-1} \tau\left(\alpha_{1} \cdot \alpha_{2} \otimes u\right)\right)
\end{aligned}
$$

for $\alpha_{1}, \alpha_{2}, \alpha_{3} \in T^{*}$ and $u \in S_{0}^{2} T^{*}$.

Let $\tilde{P}_{j}: \mathscr{F}_{j} \rightarrow \mathscr{F}_{j+1}$ be the differential operator determined by

$$
\varphi_{j+1} \tilde{P}_{j}=D_{j} \varphi_{j}
$$

for $0 \leqslant j \leqslant n-1$. We now verify that $\tilde{P}_{j}=P_{j}$ for $0 \leqslant j \leqslant n-1$. We begin by showing that

$$
\sigma\left(\tilde{P}_{j}\right)=\sigma\left(P_{j}\right), \quad 0 \leqslant j \leqslant n-1 .
$$

According to the definition of $D_{0}$, the diagram

$$
\begin{array}{lll}
\mathscr{R}_{2} & \stackrel{\nabla}{\rightarrow} & \mathscr{T}^{*} \otimes \mathscr{R}_{2} \\
\downarrow \pi_{0} & & \downarrow \rho_{0} \\
\mathscr{T} & \stackrel{D_{0}}{\rightarrow} & \mathscr{E}^{1}
\end{array}
$$

is commutative. Thus by the commutativity of (7.6), the diagram

$$
\begin{array}{lll}
\mathscr{R}_{2} & \stackrel{\nabla}{\rightarrow} & \mathscr{T}^{*} \otimes \mathscr{R}_{2} \\
\downarrow \pi_{0} & & \downarrow \stackrel{1}{2} p_{0} \psi \pi_{0} \\
\mathscr{T} & \stackrel{\check{P}_{0}}{\rightarrow} & S_{0}^{2} \mathscr{T}^{*}
\end{array}
$$

also commutes. Therefore, since the symbol of the operator $\nabla$ of the above diagram is equal to the identity mapping of $T^{*} \otimes R_{2}$, the symbol of $\tilde{P}_{0}$ is given by $\sigma\left(\tilde{P}_{0}\right)=\frac{1}{2} p_{0} \psi=\sigma\left(P_{0}\right)$. By the definition of $\tilde{P}_{n-1}$, the diagram

$$
\begin{array}{ccc}
\wedge^{n} \mathscr{T}^{*} \otimes S_{0}^{2} \mathscr{T}^{*} & \stackrel{\tilde{P}_{n-1}}{\rightarrow} & \wedge^{n} \mathscr{T}^{*} \otimes \mathscr{T}^{*} \\
\downarrow(\text { id } \otimes \bar{\theta}) \cdot \operatorname{Tr}^{n-1} & & \downarrow \text { id } \otimes \bar{\theta} \\
\bigwedge^{n-1} \mathscr{T}^{*} \otimes g_{2} & \stackrel{\nabla^{-}}{\rightarrow} & \wedge^{n} \mathscr{T}^{*} \otimes \mathscr{R}_{2}
\end{array}
$$

commutes and so

$$
\sigma\left(\tilde{P}_{n-1}\right)=\sigma\left(\tilde{\nabla}^{\wedge}\right) \cdot \operatorname{Tr}^{n-1}=\sigma\left(P_{n-1}\right) .
$$

As in $\S 6$, let $\lambda: \wedge^{2} T^{*} \otimes T \rightarrow T^{*} \otimes \bar{g}_{1}$ be the inverse of the isomorphism $\delta: T^{*} \otimes \bar{g}_{1} \rightarrow \wedge^{2} T^{*} \otimes T$, and let

$$
\kappa: T^{*} \otimes \bar{g}_{1} \rightarrow T^{*} \otimes R_{2}^{0}
$$


be a morphism of vector bundles such that $\pi_{1} \kappa=$ id. We set $\nu_{0}=\kappa \lambda$; then $\pi_{1} \nu_{0}=\lambda$. Since $H^{2, n-2}\left(g_{1}\right)=0$, the morphism

$$
\delta: \bigwedge^{n-2} T^{*} \otimes g_{2} \rightarrow \bigwedge^{n-1} T^{*} \otimes g_{1}
$$

is injective; therefore there exists a unique morphism $\nu_{1}: M_{1}^{n-1} \rightarrow \wedge^{n-2} T^{*} \otimes g_{2}$ such that $\delta \nu_{1}=$ id. According to the commutativity of diagram (2.5) of [7] and formula (2.11) of [7], the diagram

$$
\begin{array}{ccc}
\bigwedge^{n-2} T^{*} \otimes g_{2} & \stackrel{\delta}{\rightarrow} & \bigwedge^{n-1} T^{*} \otimes g_{1} \\
\uparrow \text { id } \otimes \bar{\theta} & \uparrow \mathrm{id} \otimes \iota \\
\delta\left(\bigwedge^{n-3} T^{*} \otimes S^{2} T^{*}\right) & \stackrel{(-1)^{n} \hat{\phi}}{\rightarrow} & E_{n-2}
\end{array}
$$

is commutative; therefore, for $u \in E_{n-2}$ we have

$$
\nu_{1}(\mathrm{id} \otimes \iota) u=(-1)^{n}(\mathrm{id} \otimes \bar{\theta}) \cdot \hat{\phi}^{-1} u .
$$

Assume that $n \geqslant 4$. Let $Q: \mathscr{T}^{*} \otimes \mathscr{R}_{1} \rightarrow \mathscr{C}^{2,2}$ be the differential operator of order 2 defined by

$$
Q u=\nabla^{\wedge}\left(u+\nu_{0} \pi_{0} \nabla^{\wedge} u\right)
$$

for $u \in \mathscr{T}^{*} \otimes \mathscr{R}_{1}$; the commutativity of diagram (3.10) tells us that the diagram

$$
\begin{array}{ccc}
\mathscr{T}^{*} \otimes \mathscr{R}_{2} & \stackrel{Q}{\rightarrow} & \mathscr{C}^{2,2} \\
\downarrow \rho_{0} & & \downarrow \rho_{1} \\
\mathscr{E}^{1} & \stackrel{D_{1}}{\rightarrow} & \mathscr{E}^{2}
\end{array}
$$

commutes. By (7.14) and the commutativity of (7.6), we now see that this implies that the diagram

$$
\begin{aligned}
& \mathscr{T}^{*} \otimes \mathscr{R}_{2} \quad \stackrel{Q}{\rightarrow} \quad \mathscr{C}^{2,2} \\
& \downarrow p_{0} \psi \pi_{0} \quad \downarrow \rho_{1} \\
& S_{0}^{2} \mathscr{T}^{*} \quad \stackrel{\tilde{P}_{1}}{\rightarrow} \quad \mathscr{E}_{1}^{0} \quad \stackrel{\varpi}{\rightarrow} \quad \mathscr{E}^{2}
\end{aligned}
$$

is also commutative. According to (3.11) and Lemma 6.1, since $\pi_{1} \nu_{0}=\lambda$, we have

$$
\begin{aligned}
\pi_{1} \sigma(Q)\left(\alpha_{1} \cdot \alpha_{2} \otimes u\right) & =\alpha_{1} \wedge \lambda\left(\alpha_{2} \wedge \pi_{0} u\right)+\alpha_{2} \wedge \lambda\left(\alpha_{1} \wedge \pi_{0} u\right) \\
& =(\mathrm{id} \otimes \iota) \tau\left(\alpha_{1} \cdot \alpha_{2} \otimes \psi \pi_{0} u\right)
\end{aligned}
$$

for $\alpha_{1}, \alpha_{2} \in T^{*}$ and $u \in T^{*} \otimes R_{2}$. Hence from the commutativity of (7.17), we deduce that

$$
\varpi \sigma\left(\tilde{P}_{1}\right)\left(\alpha_{1} \cdot \alpha_{2} \otimes p_{0} h\right)=\varpi \tau\left(\alpha_{1} \cdot \alpha_{2} \otimes h\right)
$$

for $\alpha_{1}, \alpha_{2} \in T^{*}$ and $h \in S^{2} T^{*}$. The above characterization of the orthogonal projection $\tilde{\rho}_{1}$ therefore implies that

$$
\sigma\left(\tilde{P}_{1}\right)\left(\alpha_{1} \cdot \alpha_{2} \otimes p_{0} h\right)=\tilde{\rho}_{1} \tau\left(\alpha_{1} \cdot \alpha_{2} \otimes h\right)
$$


for $\alpha_{1}, \alpha_{2} \in T^{*}$ and $h \in S^{2} T^{*}$, and so by (7.11) we see that (7.15) holds for $j=1$.

For $2 \leqslant j \leqslant n-3$, if $\alpha \in T^{*}$ and $u \in \wedge^{j} T^{*} \otimes g_{1}$ with $\delta u=0$, it is easily seen that

$$
\sigma\left(D_{j}\right): T^{*} \otimes E^{j} \rightarrow E^{j+1}
$$

sends $\alpha \otimes \gamma_{3} u$ into $\gamma_{3}(\alpha \wedge u)$. Hence by (7.14), we have

$$
\begin{aligned}
\varphi_{j+1} \sigma\left(\tilde{P}_{j}\right)(\alpha \otimes u) & =\sigma\left(D_{j}\right)\left(\alpha \otimes \varphi_{j} u\right)=2 \gamma_{3}(\mathrm{id} \otimes \iota)(\alpha \wedge u) \\
& =\varphi_{j+1}(\alpha \wedge u)
\end{aligned}
$$

for $\alpha \in T^{*}$ and $u \in E_{j-1}^{0}$; by the characterization of $\tilde{\rho}_{j}$, we infer that

$$
\sigma\left(\tilde{P}_{j}\right)(\alpha \otimes u)=\tilde{\rho}_{j}(\alpha \wedge u)
$$

for $\alpha \in T^{*}$ and $u \in E_{j-1}^{0}$. Thus we have shown that (7.15) holds for $2 \leqslant j \leqslant n-3$.

Let $Q: \mathscr{C}^{2, n-2} \rightarrow \mathscr{H}^{2, n-1}\left(g_{1}\right)$ be the differential operator of order 2 defined by

$$
Q u=\nabla^{\wedge}\left(u+\nu_{1} \pi_{1} \nabla^{\wedge} u\right)
$$

for $u \in \mathscr{C}^{2, n-2}$. The commutativity of diagram (3.10) tells us that the diagram

$$
\begin{array}{cc}
\mathscr{C}^{2, n-2} & \stackrel{Q}{\rightarrow} \\
\downarrow \rho_{1} & \nearrow D_{n-2} \\
\mathscr{E}^{n-2} &
\end{array}
$$

commutes. From formula (3.11), we deduce that

$$
\sigma\left(D_{n-2}\right)\left(\alpha_{1} \cdot \alpha_{2} \otimes \gamma_{3} u\right)=\alpha_{1} \wedge \nu_{1}\left(\alpha_{2} \wedge u\right)+\alpha_{2} \wedge \nu_{1}\left(\alpha_{1} \wedge u\right)
$$

for $\alpha_{1}, \alpha_{2} \in T^{*}$ and $u \in \wedge^{n-2} T^{*} \otimes g_{1}$ with $\delta u=0$. By (7.18) and (7.16), the symbol of

is determined by

$$
\tilde{P}_{n-2}: \mathscr{E}_{n-3}^{0} \rightarrow \wedge^{n} \mathscr{T}^{*} \otimes S_{0}^{2} \mathscr{T}^{*}
$$

$$
\begin{aligned}
2(\mathrm{id} & \otimes \bar{\theta}) \operatorname{Tr}^{n-1} \cdot \sigma\left(\tilde{P}_{n-2}\right)\left(\alpha_{1} \cdot \alpha_{2} \otimes u\right) \\
& =\alpha_{1} \wedge \nu_{1}\left(\alpha_{2} \wedge(\mathrm{id} \otimes \iota) u\right)+\alpha_{2} \wedge \nu_{1}\left(\alpha_{1} \wedge(\mathrm{id} \otimes \iota) u\right) \\
& =(-1)^{n}(\mathrm{id} \otimes \bar{\theta})\left(\alpha_{1} \wedge \hat{\phi}^{-1}\left(\alpha_{2} \wedge u\right)+\alpha_{2} \wedge \hat{\phi}^{-1}\left(\alpha_{1} \wedge u\right)\right)
\end{aligned}
$$

for $\alpha_{1}, \alpha_{2} \in T^{*}$ and $u \in E_{n-3}^{0}$; thus we see that

$$
\operatorname{Tr}^{n-1} \cdot \sigma\left(\tilde{P}_{n-2}\right)\left(\alpha_{1} \cdot \alpha_{2} \otimes u\right)=\frac{(-1)^{n}}{2}\left(\alpha_{1} \wedge \hat{\phi}^{-1}\left(\alpha_{2} \wedge u\right)+\alpha_{2} \wedge \hat{\phi}^{-1}\left(\alpha_{1} \wedge u\right)\right) \text {. }
$$

From (7.12), we infer that (7.15) holds for $j=n-2$.

Finally, assume that $n=3$. Let

$$
Q: \mathscr{T}^{*} \otimes \mathscr{R}_{2} \rightarrow \mathscr{H}^{2.2}\left(g_{1}\right)
$$

be the differential operator of order 3 defined by

$$
Q u=\nabla^{\wedge}\left(u+\nu_{0} \pi_{0} \nabla^{\wedge} u+\nu_{1} \pi_{1} \nabla^{\wedge} u+\nu_{1} \pi_{1} \nabla^{\wedge} \nu_{0} \pi_{0} \nabla^{\wedge} u\right)
$$

for $u \in \mathscr{T}^{*} \otimes \mathscr{R}_{1}$; the commutativity of diagram (3.10) tells us that the diagram

$$
\begin{array}{ccc}
\mathscr{T}^{*} \otimes \mathscr{R}_{2} & \stackrel{Q}{\rightarrow} & \mathscr{E}^{2} \\
\downarrow \rho_{0} & \nearrow D_{1} & \\
\mathscr{E}^{1} & &
\end{array}
$$


commutes. By (7.14) and the commutativity of (7.6), we now see that the diagram

$$
\begin{array}{ccc}
\mathscr{T}^{*} \otimes \mathscr{R}_{2} & \stackrel{Q}{\rightarrow} & \mathscr{E}^{2} \\
\downarrow \frac{1}{2} p_{0} \psi \pi_{0} & & \uparrow(\mathrm{id} \otimes \bar{\theta}) \cdot \operatorname{Tr}^{2} \\
S_{0}^{2} \mathscr{T}^{*} & \stackrel{\tilde{P}_{1}}{\rightarrow} & \wedge^{3} \mathscr{T}^{*} \otimes S_{0}^{2} \mathscr{T}^{*}
\end{array}
$$

also commutes. Since $\pi_{1} \nu_{0}=\lambda$, by formula (3.12) and Lemma 6.1, we have

$$
\begin{aligned}
& \sigma(Q)\left(\alpha_{1} \cdot \alpha_{2} \cdot \alpha_{3} \otimes u\right) \\
& =\frac{1}{2} \sum_{p \in \mathbb{S}} \alpha_{p(1)} \wedge \nu_{1}\left(\alpha_{p(2)} \wedge \lambda\left(\alpha_{p(3)} \wedge \pi_{0} u\right)+\alpha_{p(3)} \wedge \lambda\left(\alpha_{p(2)} \wedge \pi_{0} u\right)\right) \\
& =\alpha_{1} \wedge \nu_{1}(\mathrm{id} \otimes \iota) \tau\left(\alpha_{2} \cdot \alpha_{3} \otimes \psi \pi_{0} u\right)+\alpha_{2} \wedge \nu_{1}(\mathrm{id} \otimes \iota) \tau\left(\alpha_{1} \cdot \alpha_{3} \otimes \psi \pi_{0} u\right) \\
& \quad+\alpha_{3} \wedge \nu_{1}(\mathrm{id} \otimes \iota) \tau\left(\alpha_{1} \cdot \alpha_{2} \otimes \psi \pi_{0} u\right)
\end{aligned}
$$

for $\alpha_{1}, \alpha_{2}, \alpha_{3} \in T^{*}$ and $u \in T^{*} \otimes R_{2}$. Hence from the commutativity of (7.19) and by (7.16), we deduce that

$$
\begin{aligned}
& \frac{1}{2}(\mathrm{id} \otimes \bar{\theta}) \cdot \operatorname{Tr}^{2} \cdot \sigma\left(\tilde{P}_{1}\right)\left(\alpha_{1} \cdot \alpha_{2} \cdot \alpha_{3} \otimes p_{0} h\right) \\
&=-(\mathrm{id} \otimes \bar{\theta})\left(\alpha_{1} \wedge\right. \hat{\phi}^{-1} \tau\left(\alpha_{2} \cdot \alpha_{3} \otimes h\right) \\
&\left.+\alpha_{2} \wedge \hat{\phi}^{-1} \tau\left(\alpha_{1} \cdot \alpha_{3} \otimes h\right)+\alpha_{3} \wedge \hat{\phi}^{-1} \tau\left(\alpha_{1} \cdot \alpha_{2} \otimes h\right)\right)
\end{aligned}
$$

for $\alpha_{1}, \alpha_{2}, \alpha_{3} \in T^{*}$ and $h \in S^{2} T^{*}$, and hence by (7.13) that (7.15) holds for $j=1$ and $n=3$.

From (7.15), it follows that the differential operator $\tilde{P}_{j}-P_{j}: \mathscr{F}_{j} \rightarrow \mathscr{F}_{j+1}$ is of order $r_{j+1}-r_{j}$ for $0 \leqslant j \leqslant n-1$. According to $\$ \S 5,6$ of [7] and the commutativity of diagram (12.1) of [7], the complex (7.10) satisfies the hypotheses of Theorem 0.2(ii) of [7], and hence the morphism

$$
p\left(j_{r_{j+1}-r_{j}} \circ P_{j-1}\right): J_{r_{j+1}-r_{j-1}+1}\left(F_{j-1}\right) \rightarrow J_{r_{j+1}-r_{j}}\left(F_{j}\right)
$$

is surjective for $1 \leqslant j \leqslant n$. We now verify by induction on $j \geqslant 0$ that $\tilde{P}_{j}=P_{j}$. First, since $\pi_{0}: R_{2} \rightarrow T$ and the mappings (1.15) are surjective, and since the operator $\tilde{P}_{0}-P_{0}$ of order 0 vanishes on $\Theta$, we see that $\tilde{P}_{0}=P_{0}$. Suppose that $j \geqslant 1$ and $\tilde{P}_{j-1}=P_{j-1}$. If $x \in X$ and $u \in \mathscr{F}_{j, x}$, by the surjectivity of (7.20), there exists $v \in \mathscr{F}_{j-1, x}$ such that

$$
j_{r_{j+1}-r_{j}}\left(u-P_{j-1} v\right)(x)=0
$$

then

$$
\left(\left(\tilde{P}_{j}-P_{j}\right) u\right)(x)=\left(\left(\tilde{P}_{j}-P_{j}\right) P_{j-1} v\right)(x)=0,
$$

since the sequences (3.18) and (7.10) are complexes; hence $\tilde{P}_{j}=P_{j}$.

From (7.14), we now deduce that

$$
\varphi_{j+1} P_{j}=D_{j} \varphi_{j}
$$

for $0 \leqslant j \leqslant n-1$. Thus (7.10) is the resolution obtained from (3.18) by means of the isomorphisms $\varphi_{j}$. Therefore according to Theorem 3.1, the exactness assertions of 
Theorems 12.1 and 12.2 of [7] hold; in particular the sequence (7.10) is exact. By Proposition 5.1, Lemma 7.2 and the relations (7.21), it follows that

$$
\left\langle P_{j} u, v\right\rangle+(-1)^{j}\left\langle u, P_{n-j-1} v\right\rangle=d \beta_{j}(u \oplus v)
$$

for $u \in \mathscr{F}_{j}$ and $v \in \mathscr{F}_{n-j-1}$, where

$$
\beta_{j}: \mathscr{F}_{j} \oplus \mathscr{F}_{n-j-1} \rightarrow \wedge^{n-1} \mathscr{T}^{*}
$$

is the differential operator of order $r_{j+1}-r_{j}$ defined by

$$
\beta_{j}(u \oplus v)=-\frac{1}{c n} \alpha_{j}\left(\varphi_{j} u \oplus \varphi_{n-j-1} v\right)
$$

for $u \in \mathscr{F}_{j}$ and $v \in \mathscr{F}_{n-j-1}$, with $c=8$ if $n \geqslant 4$ and $c=2$ if $n=3$. Here $\alpha_{j}$ is the differential operator of order $r_{j+1}-r_{j}$ given by Proposition 5.1. Thus we have essentially obtained the results of Propositions 11.1 and 11.2 of [7], although we did not derive explicit formulas for the differential operators $\beta_{j}$, as is done in [7, §11].

Consider the complex $\mathscr{F} .=\oplus_{j} \mathscr{F}_{j}$ whose differential is induced by the differential operators $P_{j}$. By (7.21), the morphisms $\varphi_{j}$ induce isomorphisms

$$
\varphi: H^{i}\left(\Gamma_{\Phi}(\mathscr{F} .)\right) \rightarrow H^{i}\left(\Gamma_{\Phi}\left(\mathscr{E}^{\bullet}\right)\right) .
$$

Since $\varphi_{0}$ is the identity mapping of $T$, the diagram

$$
\begin{array}{ccc}
H_{\Phi}^{i}(X, \Theta) & \rightarrow & H^{i}\left(\Gamma_{\Phi}(\mathscr{F} .)\right) \\
\downarrow \text { id } & & \\
H_{\Phi}^{i}(X, \Theta) & \rightarrow & H^{i}\left(\Gamma_{\Phi}\left(\mathscr{E}^{\bullet}\right)\right),
\end{array}
$$

whose horizontal arrows are the isomorphisms determined by the resolutions (7.10) and (3.18) of $\Theta$, is commutative.

Assume that $X$ is oriented. Consider the mapping sending $u \otimes v$ onto $\int_{X}\langle u, v\rangle$, with $u \in C^{\infty}\left(F_{j}\right)$ and $v \in C_{0}^{\infty}\left(F_{n-j}\right)$ satisfying $P_{j} u=0$ and $P_{n-j} v=0$, where $\langle u, v\rangle$ is given by the mapping (11.33) of [7]. By (7.22) and Stokes' theorem, this mapping induces a well-defined pairing

$$
H^{j}(\Gamma(\mathscr{F} .)) \otimes H^{n-j}\left(\Gamma_{0}(\mathscr{F} .)\right) \rightarrow \mathbf{R} .
$$

From the isomorphisms $H_{\Phi}^{i}(X, \Theta) \rightarrow H^{i}\left(\Gamma_{\Phi}(\mathscr{F}).\right)$ determined by the resolution (3.18) of $\Theta$, and the pairing (7.24), we obtain a pairing

$$
H^{j}(X, \Theta) \otimes H_{0}^{n-j}(X, \Theta) \rightarrow \mathbf{R} .
$$

The pairings (7.24) and (7.25) were considered in [7, §14], when $X$ is compact. By the commutativity of diagram (7.23), Lemma 7.2 and Theorems 1.1 and 5.1, we obtain the following result.

THEOREM 7.1. If $(X, g)$ is a conformally flat oriented Riemannian manifold of dimension $n \geqslant 3$, the pairings (1.22) and (5.14) are nondegenerate and are equal to $(-c n)$-times the pairing (7.25), where $c=8$ if $n \geqslant 4$ and $c=2$ if $n=3$.

We thus obtain a different proof of Theorem 14.1 of [7].

The isomorphisms $\varphi_{j}$ and the sequence (7.10) depend on the metric $g$, while the sequence (3.18) corresponding to $R_{2}$ depends only on the conformal structure on $X$. 
Let $\Omega^{s}$ denote the bundle of densities of order $s$ on $X$. The canonical measure $\mu_{g}$ of the Riemannian manifold $(X, g)$ is a section of $\Omega^{1}$, and $\mu_{g}^{k}$ is a nowhere vanishing section of $\Omega^{k}$. As in [7, §13], we identify the section $\tilde{g}=\mu_{g}^{-2 / n} \otimes g$ of $\Omega^{-2 / n} \otimes S^{2} T^{*}$ with the conformal class of $g$. We define isomorphisms

$$
\tilde{\varphi}_{0}: T \rightarrow E^{0}, \quad \tilde{\varphi}_{n}: F_{n} \rightarrow E^{n}, \quad \tilde{\varphi}_{j}: \Omega^{-2 / n} \otimes F_{j} \rightarrow E^{j}
$$

for $1 \leqslant j \leqslant n-1$ by setting

$$
\tilde{\varphi}_{0}=\varphi_{0}=\mathrm{id}, \quad \tilde{\varphi}_{n}=\varphi_{n}, \quad \tilde{\varphi}_{j}\left(\mu_{g}^{-2 / n} \otimes u\right)=\varphi_{j}(u)
$$

for $u \in F_{j}, 1 \leqslant j \leqslant n-1$; it is easily verified that these isomorphisms depend only on $\tilde{g}$, and that the sequence (13.14) of [7] is the one obtained from (3.18) by means of the isomorphisms $\tilde{\varphi}_{j}$. Since the complex (3.18) depends only on $R_{2}$ and the isomorphisms $\tilde{\varphi}_{j}$ depend only on $\tilde{g}$, we see that the resolution (13.14) of $\Theta$ depends only on $\tilde{g}$, as is shown also in [7, §13].

Let $\sigma: g \rightarrow g$ be the morphism determined by the metric $g$ defined by

$$
\begin{gathered}
\sigma(\xi)=\frac{1}{2 n} \bar{\theta}\left(g^{b}(\xi)\right), \quad \sigma(\bar{\theta}(\alpha))=2 n g^{\sharp}(\alpha), \\
\sigma(u)=u, \quad \sigma(\mathrm{id})=-\mathrm{id}
\end{gathered}
$$

for $\xi \in T, \alpha \in T^{*}$ and $u \in \bar{g}_{1}$. Then by (7.1), (7.2) and (5.2), it is easily verified that $\sigma_{x}$ is a Cartan involution of $g_{x}$ for $x \in X$; clearly $\sigma$ satisfies (5.15). Consider the symmetric positive definite form $\bar{B}_{\sigma}$ on $g$ and the scalar product $(, \quad)$ on $\wedge T^{*} \otimes g$ induced by it. Using (7.3), we see that

$$
\bar{B}_{\sigma \mid T}=g,
$$

and so the scalar product ( , ) on $\wedge T^{*}$ induced by $\bar{B}_{\sigma}$ is the one corresponding to g.

According to $\S 5$, we may consider the resolution (5.17) of $\Theta$, the morphisms (5.18) and, when $X$ is oriented, the isomorphisms (5.21) determined by $\sigma$. We have

$$
\begin{aligned}
& F^{0}=T, \quad F^{1}=\left\{u \in T^{*} \otimes T \mid \delta^{*} u=0\right\}, \\
& F^{j}=\left\{u \in \wedge^{j} T^{*} \otimes g_{1} \mid \delta u=0, \delta^{*} u=0\right\}, \\
& F^{n-1}=E^{n-1}, \quad F^{n}=E^{n}
\end{aligned}
$$

for $2 \leqslant j \leqslant n-2$, where $\delta^{*}$ is the adjoint of $\delta$ (with respect to $\vec{B}_{\sigma}$ ).

For $0 \leqslant j \leqslant n$, we now define isomorphisms of vector bundles $\psi_{j}: F_{j} \rightarrow F^{j}$ such that $\gamma \psi_{j}=\varphi_{j}$. Let $\psi_{0}$ be the identity mapping of $T$. Since $F^{1}$ is the orthogonal complement to $g_{1}$ in $T^{*} \otimes T$, we see that $F^{1}=\nu\left(S_{0}^{2} T^{*}\right)$ and we set $\psi_{1}=\nu$. Next, for $2 \leqslant j \leqslant n-2$, we wish to show that $F^{j}$ is equal to the image of $E_{j-1}^{0}$ under the mapping (7.7). Indeed, if $u \in \wedge^{j} T^{*} \otimes \wedge^{2} T^{*}$ and $v \in \wedge^{j-1} T^{*} \otimes T^{*}$, we set

$$
\bar{u}=(\operatorname{id} \otimes \imath) u \in \wedge^{j} T^{*} \otimes \bar{g}_{1}, \quad \bar{v}=(\operatorname{id} \otimes \bar{\theta}) v \in \wedge^{j-1} T^{*} \otimes g_{2} ;
$$

then

$$
\delta \bar{v}=(\mathrm{id} \otimes \iota) v_{1}+\omega \otimes \mathrm{id}
$$


where $v_{1} \in \wedge^{j} T^{*} \otimes \wedge^{2} T^{*}$ and $\omega \in \wedge^{j} T^{*}$. By (7.5), (7.9) and the commutativity of diagram (10.6) of [7], we have

$$
(\delta \bar{v}, \bar{u})=\left((\operatorname{id} \otimes \iota) v_{1},(\operatorname{id} \otimes \iota) u\right)=2 n\left(v_{1}, u\right)=2 n(A v, u),
$$

where

$$
A: \wedge^{j-1} T^{*} \otimes T^{*} \rightarrow\left(\wedge^{j} T^{*} \otimes \wedge^{2} T^{*}\right) \oplus \wedge^{j} T^{*}
$$

is the morphism of $\S 10$ of [7] and $(A v, u)$ is the scalar product of the elements $A v, u$ of $\left(\wedge^{j} T^{*} \otimes \wedge^{2} T^{*}\right) \oplus \wedge^{j} T^{*}$ considered there. Hence $\delta^{*} \bar{u}=0$ if and only if $A^{*} u=0$ in the sense of $\S 10$ of [7]. From the equality (10.13) of [7] and the commutativity of diagram (6.2), we deduce that

$$
(\mathrm{id} \otimes \imath) E_{j-1}^{0}=F^{j} \cap\left(\wedge^{j} T^{*} \otimes \bar{g}_{1}\right) .
$$

The desired result now follows from the fact that the mapping (7.8) is an isomorphism. We set $\psi_{j}=2(\mathrm{id} \otimes \iota)$ for $2 \leqslant j \leqslant n-1$. Finally, we let $\psi_{n-1}=\varphi_{n-1}$ and $\psi_{n}=\varphi_{n}$.

Since $\varphi_{j}=\gamma \psi_{j}$ for $0 \leqslant j \leqslant n$, we see that (7.10) is the sequence obtained from (5.17) by means of the isomorphisms $\psi_{j}$. In terms of the scalar products on the bundles $F_{j}$ considered in [7] and the scalar product on $\wedge T^{*} \otimes g$ and its sub-bundles $F^{j}$ determined by $\bar{B}_{\sigma}$, according to (7.26), (7.9) and (7.3), it is easily verified that

$$
\left(\psi_{j} u, \psi_{j} v\right)=c_{j}(u, v)
$$

for $u, v \in F_{j}$, where $c_{0}=c_{1}=1, c_{j}=c n$ for $2 \leqslant j \leqslant n-2$ and $c_{n-1}=c_{n}=(c n)^{2}$, with $c=8$ if $n \geqslant 4$ and $c=2$ if $n=3$. Set $d_{j}=-c n / c_{j}$, where $c=8$ if $n \geqslant 4$ and $c=2$ if $n=3$; then clearly we have

$$
d_{j} d_{n-j}=1 .
$$

Now suppose that $X$ is oriented and let $\Omega$ be the corresponding volume form of $(X, g)$. Consider the isomorphisms

$$
\tilde{*}: F_{j} \rightarrow F_{n-j}
$$

equal to $d_{j} \psi_{n-j}^{-1} * \psi_{j}$, where $*$ is the isomorphism (5.21). It is easily seen that

$$
\begin{gathered}
\tilde{*} \xi=\Omega \otimes g^{b}(\xi), \quad \tilde{*}(\Omega \otimes \alpha)=g^{\sharp}(\alpha), \\
\tilde{*} h=\Omega \otimes h, \quad \tilde{*}(\Omega \otimes h)=(-1)^{n-1} h
\end{gathered}
$$

for $\xi \in T, \alpha \in T^{*}$ and $h \in S_{0}^{2} T^{*}$, and that $\tilde{*}: E_{j-1}^{0} \rightarrow E_{n-j-1}^{0}$ is the restriction of

$$
* \otimes \mathrm{id}: \wedge^{j} T^{*} \otimes \wedge^{2} T^{*} \rightarrow \wedge^{n-j} T^{*} \otimes \wedge^{2} T^{*}
$$

for $2 \leqslant j \leqslant n-2$; thus we obtain a result also given by Proposition 9.1 of [7]. Therefore (7.29) is equal to the isomorphism $*$ of $\S 14$ of [7]. Since the isomorphisms (5.21) satisfy (1.24) and (1.25) for all $u, v \in F^{j}$, we see that the isomorphisms $*: F_{j} \rightarrow F_{n-j}$ satisfy (1.24) and (1.25) for all $u, v \in F_{j}$. Indeed, (1.24) follows from (7.28); if $u, v \in F_{j}$, by (7.27) we have

$$
\left\langle\psi_{j} u, * \psi_{j} v\right\rangle=\left(\psi_{j} u, \psi_{j} v\right) \Omega=c_{j}(u, v) \Omega
$$

and by Lemma 7.2

$$
\begin{aligned}
\left\langle\psi_{j} u, * \psi_{j} v\right\rangle & =\frac{1}{d_{j}}\left\langle\psi_{j} u, \psi_{n}, * v\right\rangle=-\frac{c n}{d_{j}}\langle u, * v\rangle \\
& =c_{j}\langle u, * v\rangle,
\end{aligned}
$$

and so (1.25) holds. 
From the above results and Theorem 5.2, we deduce that the sequence (7.10) (with $\Theta$ omitted) is an elliptic complex and that it is formally selfadjoint, namely that it satisfies conditions (I)-(IV) of the introduction of [7].

\section{REFERENCES}

1. M. Berger, Les espaces symétriques non compacts, Ann. Sci. École Norm. Sup. (3) 74 (1957), 85-177.

2. E. Calabi, On compact, Riemannian manifolds with constant curvature. I, Differential Geometry, Proc. Sympos. Pure Math., vol. 3, Amer. Math. Soc., Providence, R.I., 1961, pp. 155-180.

3. H. Cartan and S. Eilenberg, Homological algebra, Princeton Math. Series, No. 19, Princeton Univ. Press, Princeton, N.J., 1956.

4. C. Chevalley, Theory of Lie groups. I, Princeton Math. Series, No. 8, Princeton Univ. Press, Princeton, N.J., 1946.

5. J. Gasqui and H. Goldschmidt, Déformations infinitésimales des espaces riemanniens localement symétriques. I, Adv. in Math. 48 (1983), 205-285.

6. Théorèmes de dualité en géométrie conforme, C. R. Acad. Sci. Paris Sér. I Math. 294 (1982), 99-102, 201-203.

7. __ Déformations infinitésimales des structures conformes plates, Progress in Mathematics, Vol. 52, Birkhäuser, Boston, Basel and Stuttgart, 1984

8. H. Goldschmidt, Existence theorems for analytic linear partial differential equations, Ann. of Math.

(2) 86 (1967), 246-270.

9. Sur la structure des équations de Lie. II. Équations formellement transitives, J. Differential Geom. 7 (1972), 67-95.

10. S. Helgason, Differential geometry and symmetric spaces, Academic Press, New York, 1962.

11. S. Kobayashi, Transformation groups in differential geometry, Ergebnisse der Math. und ihrer Grenzgebiete, Band 70, Springer-Verlag, Berlin, Heidelberg and New York, 1972.

12. S. Kobayashi and T. Nagano, On filtered Lie algebras and geometric structures. I, J. Math. Mech. 13 (1964), 875-907.

13. B. Kostant, Lie algebra cohomology and the generalized Borel-Weil theorem, Ann. of Math. (2) 74 (1961), 329-387.

14. T. Matsuki, The orbits of affine symmetric spaces under the action of minimal parabolic subgroups, $\mathrm{J}$. Math. Soc. Japan 31 (1979), 331-357.

15. J. W. Milnor and J. D. Stasheff, Characteristic classes, Ann. of Math. Studies, No. 76, Princeton Univ. Press, Princeton, N.J., and Univ. of Tokyo Press, Tokyo, 1974.

16. T. Ochiai, Geometry associated with semisimple flat homogeneous spaces, Trans. Amer. Math. Soc. 152 (1970), 159-193.

17. D. C. Spencer, Overdetermined systems of linear partial differential equations, Bull. Amer. Math. Soc. 75 (1969), 179-239.

18. M. Spivak, A comprehensive introduction to differential geometry, Vol. 1, Publish or Perish, Inc., Berkeley, Calif., 1970.

19. N. Steenrod, The topology of fibre bundles, Princeton Math. Series, No. 14, Princeton Univ. Press, Princeton, N.J., 1951.

20. K. Kodaira, On deformations of some complex pseudo-group structures, Ann. of Math. (2) 71 (1960), $224-302$.

21. K. Kodaira and D. C. Spencer, On deformations of complex analytic structures. I, II, Ann. of Math. (2) 67 (1958), 328-466.

22. J. Lafontaine, Modules de structures conformes plates et cohomologie de groupes discrets, C. R. Acad. Sc. Paris Sér. I Math. 297 (1983), 655-658.

23. D. C. Spencer, Deformation of structures on manifolds defined by transitive, continuous pseudogroups. I, II, Ann. of Math. (2) 76 (1962), 306-445.

School of Mathematics, Institute for Advanced Study, Princeton, New Jersey 08540

Department of Mathematics, Columbia University, New York, New York 10027 